Florida International University

FIU Digital Commons

$11-13-2020$

\title{
Digital and Mixed Domain Hardware Reduction Algorithms and Implementations for Massive MIMO
}

\author{
Najath A. Mohomed \\ Florida International University, nmoho001@fiu.edu
}

Follow this and additional works at: https://digitalcommons.fiu.edu/etd

Part of the Digital Circuits Commons, Electrical and Electronics Commons, Hardware Systems

Commons, Signal Processing Commons, Theory and Algorithms Commons, and the VLSI and Circuits,

Embedded and Hardware Systems Commons

\section{Recommended Citation}

Mohomed, Najath A., "Digital and Mixed Domain Hardware Reduction Algorithms and Implementations for Massive MIMO" (2020). FIU Electronic Theses and Dissertations. 4548.

https://digitalcommons.fiu.edu/etd/4548

This work is brought to you for free and open access by the University Graduate School at FIU Digital Commons. It has been accepted for inclusion in FIU Electronic Theses and Dissertations by an authorized administrator of FIU Digital Commons. For more information, please contact dcc@fiu.edu. 


\section{FLORIDA INTERNATIONAL UNIVERSITY \\ Miami, Florida}

\section{DIGITAL AND MIXED DOMAIN HARDWARE COMPLEXITY REDUCTION ALGORITHMS AND IMPLEMENTATIONS FOR MASSIVE MIMO}

A dissertation submitted in partial fulfillment of the requirements for the degree of DOCTOR OF PHILOSOPHY in ELECTRICAL AND COMPUTER ENGINEERING by Najath Akram Mohomed 


\section{To: Dean John Volakis}

College of Engineering and Computing

This dissertation, written by Najath Akram Mohomed, and entitled Digital and Mixed Domain Hardware Complexity Reduction Algorithms and Implementations for Massive MIMO, having been approved in respect to style and intellectual content, is referred to you for judgment.

We have read this dissertation and recommend that it be approved.

Elias Alwan

Shubhendu Bhardwaj

Todd Crowl

Arjuna Madanayake, Major Professor

Date of Defense: November 13, 2020

The dissertation of Najath Akram Mohomed is approved.

Dean John Volakis

College of Engineering and Computing

Andrés G. Gil

Vice President for Research and Economic Development and Dean of the University Graduate School

Florida International University, 2020 
(c) Copyright 2020 by Najath Akram Mohomed All rights reserved. 


\title{
DEDICATION
}

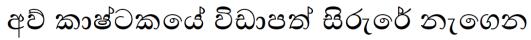

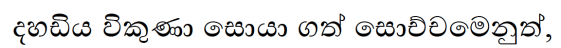

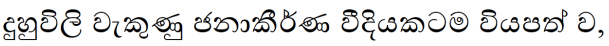

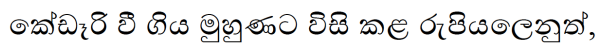

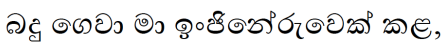

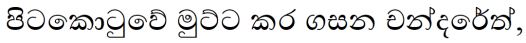

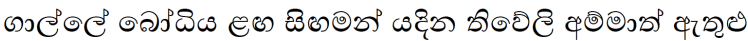

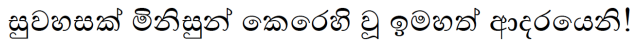

To the people in Sri Lanka! 


\section{ACKNOWLEDGMENTS}

First and foremost, my sincere gratitude goes to my advisor, Dr.Arjuna Madanayake. It would not have been possible to complete my research studies without his encouraging guidance and regular mentorship. My gratitude also extends to my dissertation committee, Dr. Elias Alwan, Dr. Shubhendu Bhardwaj and Dr. Todd Crowl for being a source of inspiration.

I would also like to express my sincere thanks to Dr. Satheesh Venkatakrishnan, Dr. John Volakis, Dr. Renato Cintra, Dr. Leonid Belostotski, Dr. Soumyajit Mandal, and Dr. Ted Rappaport for their unwavering support of my research, which also lead to several collaborative publications. I am overwhelmed in all humbleness to acknowledge all the faculty members at Florida International University, The University of Akron, and University of Ruhuna (Sri Lanka), as well as all the teachers in my life who helped me in becoming the person I am today. I would like to extend my gratitude to Dr. Viduneth Ariyarathna for his immense support in collaborative research as well as for being an amazing friend. I would also like to thank all past and present fellow researchers at the RF, Analog, and

Digital laboratory, for their support and collaboration throughout my studies.

None of my attempts at any level of my education would not have been successful without the unfaltering efforts of my beloved parents. I am also thankful to my sister who has always provided encouragement and constant support. Without

them, I would never have been able to pursue my doctoral studies.

I am extremely grateful for the financial support provided by the National Science Foundation (NSF) and Defense Advanced Research Projects Agency (DARPA) throughout my studies. Finally, I am indebted to the people of Sri Lanka and the United States, for sponsoring my education from grade one to my doctoral degree. 


\title{
ABSTRACT OF THE DISSERTATION \\ DIGITAL AND MIXED DOMAIN HARDWARE COMPLEXITY REDUCTION ALGORITHMS AND IMPLEMENTATIONS FOR MASSIVE MIMO \\ by
}

\author{
Najath Akram Mohomed \\ Florida International University, 2020 \\ Miami, Florida \\ Professor Arjuna Madanayake, Major Professor
}

Emerging 5G- and 6G- based wireless communications systems largely rely on multipleinput-multiple-output (MIMO) systems to reduce inherently extensive path losses and to facilitate high data rates and high spatial diversity. Massive MIMO systems used in mmWave and sub- $\mathrm{THz}$ applications consists of hundreds or perhaps thousands of antenna elements at base stations. Digital beamforming techniques provide the highest flexibility as well as better degrees of freedom for phased antenna arrays as compared to its analog and hybrid alternatives but has the highest hardware complexity.

Conventional digital beamformers at the receiver require a dedicated analog-todigital converter (ADC) for every antenna element, leading to $N$ ADCs for $N$ elements. The number of ADCs is the key factor determining the power consumption of an antenna array system. The digital hardware consists of fast Fourier transform (FFT) cores with a multiplier complexity of $\left(N \log _{2} N\right)$ for a system of $N$ elements in order to generate multiple beams. It is required to reduce the mixed and digital hardware complexities in MIMO systems to reduce the cost and the power consumption, while maintaining high performance.

The well-known $\Sigma-\Delta$ concept has been in use for ADCs to achieve reduced complexity. This dissertation explores an extension of the $\Sigma-\Delta$ architecture to multi- 
dimensional domain in order to implement a single $N$-port ADC to replace $N$ ADCs in an $N$-element system, using the correlation of received signals in the spatial domain. This concept has applications in conventional uniform linear arrays (ULAs) as well as in focal plane array (FPA) receiver systems.

Our analysis has shown that sparsity in the spatio-temporal frequency domain can be exploited to reduce the number of ADCs from $N$ to $\frac{N}{K}$ where $K \in \mathbb{Z}^{+}$. By using the limited field of view of practical antennas, multiple sub-arrays are combined without interferences to achieve a factor of $K$ increment in the information carrying capacity of the $\frac{N}{K}$-ADC systems. Applications of this concept include ULAs and rectangular array systems. Experimental verifications were done for a 16-element, 1.8 - 2.1 GHz wideband array system to sample using 8-ADCs.

This dissertation proposes that frequency division multiplexing (FDM) receiver outputs at an intermediate frequency (IF) can pack multiple $(M)$ narrowband channels with a guard band to avoid interference. The combined output is then sampled using a single wideband ADC and baseband channels are retrieved in the digital domain. Measurement results were obtained by employing a four-element, $28 \mathrm{GHz}$ antenna array system that combines $M=4$ channels to achieve a $75 \%$ reduction of ADC requirement.

Implementation of FFT cores in the digital domain is not always exact because the level of precision in this domain is finite. Therefore, this dissertation explores the possibility of approximating the discrete Fourier transform (DFT) matrix to achieve reduced hardware complexities at an allowable cost of accuracy. A 1024-point approximate DFT (ADFT) core was implemented on digital hardware using Radix -32 to achieve savings in cost, size, weight and power (C-SWaP) and synthesized for application specific integrated circuits (ASIC) at 45-nm technology. 


\section{TABLE OF CONTENTS}

1. INTRODUCTION . . . . . . . . . . . . . . . . . . 1

1.1 Challenges Associated with mmWave Communication . . . . . . . . . . 3

1.2 Motivation . . . . . . . . . . . . . . . . . . . . 7

1.3 Contributions and the Outline of the Dissertation . . . . . . . . . 9

1.3.1 Reduction of Mixed Domain/ADC Complexity . . . . . . . . . . . . 9

1.3.2 Reduction of Digital Hardware Complexity . . . . . . . . . . . . . . 12

1.4 Scientific Collaborations . . . . . . . . . . . . . . . . . . 12

2. MULTI-DIMENSIONAL SIGNAL PROPERTIES . . . . . . . . . . . 14

2.1 mmWave MIMO . . . . . . . . . . . . . . . . . . . . . . . . . 15

2.2 Antenna Diversity . . . . . . . . . . . . . . . . . . . . . . . 17

2.2 .1 Receiver . . . . . . . . . . . . . . . . . . . . 17

2.2 .2 Transmitter . . . . . . . . . . . . . . . . . . . . . . . . . . 19

2.3 Massive MIMO . . . . . . . . . . . . . . . . . . . . . . . . . . . . . 20

2.3.1 Spatial Multiplexing in Massive MIMO . . . . . . . . . . . . . . . 23

2.3.2 Downlink Data Transmission . . . . . . . . . . . . . . . . . . . 25

2.3.3 Importance of Beamforming with Measured Channels . . . . . . . . . . 26

2.3.4 Pilot Contamination . . . . . . . . . . . . . . . . . . . 27

2.3.5 Holographic Beamforming . . . . . . . . . . . . . . . . . . . 27

2.3.6 Limitations of Independent Rayleigh Fading Model . . . . . . . . . . 28

2.4 EM Characteristics of Planar Wave Propagation . . . . . . . . . . . . 30

2.5 MD Properties of ULAs and Rectangular Arrays . . . . . . . . . . . . . . 34

2.5.1 Region of Support . . . . . . . . . . . . . . . . . . 37

2.5 .2 Practical Aspects . . . . . . . . . . . . . . . . . . . . . 38

2.6 Spatio-temporal Filter Implementations . . . . . . . . . . . . . . . . . 38

2.7 Digital Beamforming . . . . . . . . . . . . . . . . . . . . 40

2.7 .1 FFT and TTD Beamforming . . . . . . . . . . . . . 41

3. MD SPACE-TIME $\Sigma-\Delta$ MULTIPORT CIRCUITS . . . . . . . . . 43

3.1 Background . . . . . . . . . . . . . . . . . . . . . . . . . . . 44

$3.2 \Sigma-\Delta$ Concept in MD Signal Processing for ULAs . . . . . . . . . . 46

3.2.1 Relaxation of Spatial Oversampling Requirement in MD $\Sigma-\Delta$ ADCs. $\quad 50$

3.2.2 Implementation of the Proposed MD $\Sigma-\Delta$ Architecture . . . . . . . 53

3.2 .3 Applications of the Proposed Architecture . . . . . . . . . . . . 56

3.3 Conclusion . . . . . . . . . . . . . . . . . . . . . 57

4. $\Sigma-\Delta$ CONCEPT IN MD SIGNAL PROCESSING FOR PARABOLIC DISH ANTENNA ARRAYS . . . . . . . . . . . . . 58

4.1 Background . . . . . . . . . . . . . . . . . . . . . . . . . . 59

4.1.1 Spatio-temporal $\Sigma-\Delta \mathrm{ADC} \ldots \ldots \ldots \ldots$ 
4.1 .2 Simulation Results . . . . . . . . . . . . . . . . . . . . . . 61

4.1 .3 Noise Shaping . . . . . . . . . . . . . . . . . . . . . . . . . . . 61

4.1.4 Improvements in Signal-to-Noise Ratio . . . . . . . . . . . . . . . 62

4.2 Conclusion . . . . . . . . . . . . . . . . . . . . . 63

5. ANALOG MULTIPORT NETWORK ALGORITHMS . . . . . . . . . . 64

5.1 Theoretical Analysis of AMNA _. . . . . . . . . . . . . . . 64

5.1 .1 Mathematical Analysis . . . . . . . . . . . . . . . . . . 66

5.1.2 Extension to $K$ Bands with Different Linear Transforms . . . . . . . . 68

5.1 .3 Simulated Example: ULA . . . . . . . . . . . . . . . . . . . . . 69

5.2 Extension to Rectangular Apertures . . . . . . . . . . . . . . 70

5.2 .1 Simulated Example: URA . . . . . . . . . . . . . . . . . . . 71

5.3 Hardware Implementation $\ldots \ldots \ldots 72$

5.4 Conclusion . . . . . . . . . . . . . . . . . . . . . . . 73

6. RECEIVER APPROACHES BASED ON DIFFERENT MULTIPLEXING METHODS . . . . . . . . . . . . . . . . . . 74

6.1 Design of the FDM Array Receiver . . . . . . . . . . . . . . 77

6.1 .1 Multi-Stage Down-Conversion . . . . . . . . . . . . . . . . 77

6.1.2 Phase Synchronization and Frequency Locking . . . . . . . . . . . . 78

6.1 .3 DDC and FIR Filtering . . . . . . . . . . . . . . . . 81

6.1 .4 Test-Bed Validation . . . . . . . . . . . . . . . . . 83

6.1.5 Digital FDM Multi-Beam Measurements . . . . . . . . . . . . . . 85

6.2 Conclusion . . . . . . . . . . . . . . . . . . . . . . . . . . . . . 89

7. USE OF ADFT IN DIGITAL BEAMFORMING TO REDUCE HARDWARE COMPLEXITY ......................... 91

7.1 Review of the DFT and FFT _ . . . . . . . . . . . . . . . 94

7.1.1 DFT Mathematical Definition . . . . . . . . . . . . . . . . 94

$7.1 .2 \quad$ FFT Algorithms . . . . . . . . . . . . . . . . . . . . . . . 95

7.1.3 Matrix Representation of the $N^{2}$-point DFT in Terms of the $N$-point

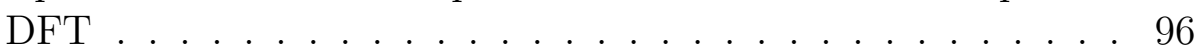

7.2 Multiplierless 32-point ADFT . . . . . . . . . . . . . . . . . . . . . 98

7.2 .1 Matrix Representation . . . . . . . . . . . . . . . . . . . . . 98

7.2 .2 Arithmetic Complexity . . . . . . . . . . . . . . . . . . . . . . 100

7.2 .3 Error Analysis . . . . . . . . . . . . . . . . . . . . . . . . 101

7.3 Approximations for the 1024-point DFT . . . . . . . . . . . . . 101

7.3.1 Approximation Methodology . . . . . . . . . . . . . . . . . . . 101

7.3.2 Arithmetic Complexity . . . . . . . . . . . . . . . . . . . . . . . . 104

7.3.3 Performance of the Proposed Approximations . . . . . . . . . . . . 105

7.4 Digital VLSI Realization . . . . . . . . . . . . . . . . . . . . . 107

7.4.1 Transpose Buffer and Twiddle Factors . . . . . . . . . . . . . . 108

7.4 .2 Circuit Complexity . . . . . . . . . . . . . . . . . . . . . . 109 
7.4.3 ASIC Synthesis and Place-Route Results: 65nm CMOS . . . . . . . . . 111

7.4.4 Analysis of the Results . . . . . . . . . . . . . . . . . . . . . . 111

7.5 Applications in Wireless Modulation . . . . . . . . . . . . . 113

7.5 .1 OFDM . . . . . . . . . . . . . . . . . . . 113

7.5.2 Multi-Beam Beamforming . . . . . . . . . . . . . . . 116

7.6 Conclusion . . . . . . . . . . . . . . . . . . . . . . . . 117

8. CONCLUSIONS AND FUTURE WORK . . . . . . . . . . . 120

BIBLIOGRAPHY . . . . . . . . . . . . . . . . . . . 124

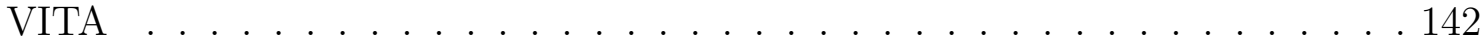




\section{LIST OF FIGURES}

FIGURE

PAGE

1.1 The utilization of available radio frequency spectrum. . . . . . . . . . 2

1.2 Propagation effects of mmWave communication. . . . . . . . . . . . 3

1.3 Antenna beams for (a) 4-element, (b) 8-element, (c) 16-element, and (d) 32 -element ULAs. . . . . . . . . . . . . . . . 6 6

1.4 Phased array receiver system architecture. . . . . . . . . . . . 8

2.1 Analog beamforming model consists of low noise amplifiers (LNAs), power amplifiers (PAs), low pass filters (LPFs), and local oscillators $(\mathrm{LOs}) \ldots \ldots \ldots \ldots \ldots \ldots$

2.2 Hybrid beamforming model consisting of analog and beamforming components. . . . . . . . . . . . . . . 16

2.3 Overview of MIMO architecture: (a) point-to-point MIMO, (b) MUMIMO, and (c) massive MIMO. . . . . . . . . . . . . . . . . . 21

2.4 (a) Conjugate beamforming for downlink transmission, and (b) matched filtering for uplink transmission in massive MIMO. . . . . . . . . . . 26

2.5 (a) Holographic beamformer with PESA, and (b) holographic massive MIMO realization using $K$ PESAs. . . . . . . . . . . . . . . . 27

2.6 (a) Locations for uncorrelation from three antennas in a ULA. (b) ULAs with $\frac{\lambda}{2}$ spacing. (c) A rectangular array with vertical and horizontal spacing of $\frac{\lambda}{2}$.

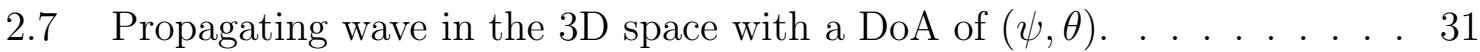

2.8 (a) Spatial domain, (b) 2D spatio-temporal, and (c) 2D continuoustime-discrete-space frequency domain illustration of a planar wave impinging on a ULA. . . . . . . . . . . . . . . . . .

2.9 (a) The spatio-temporal illustration of a 3D planar wave impinging on a planar surface $(z=0)$ and $(b)$ the 3D frequency domain representation. (c) The spatio-temporal illustration of a 2D planar wave impinging on a line $(y=0, z=0)$ and $(\mathrm{d})$ the $2 \mathrm{D}$ frequency domain

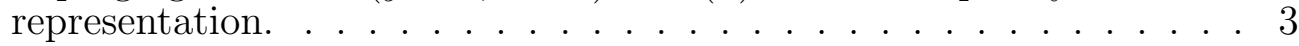

2.10 MD filtering for a (a) wideband (b) narrowband ULA. Received MD spectrum for a ULA in (c) RF and (d) downsampled at baseband. . . 38

2.11 (a) Receive mode model for an $N$-element ULA with a wave impinging at DoA of $\psi$ and (b) the TTD and sum beamforming architecture. 
3.1 (a) Architecture of a delta modulator; quantization using a delta modulator for (b) slowly varying signals and (c) rapidly varying signals. . 43

3.2 Comparison among the operation and quantization noise of (a) Nyquist sampling with traditional ADCs, (b) oversampling with traditional ADCs and (c) oversampling with $\Sigma-\Delta$ modulation. (d) First order $\Sigma-\Delta$ ADC . . . . . . . . . . . . . . . . .

3.3 (a) 1D signal (low pass) and noise (high pass) transfer functions; (b) 2D space-time transfer function for the desired plane-wave signal; (c) 2D space-time transfer function for the unwanted quantization noise. . .

3.4 a) Feedback circuit for a conventional first-order $\Sigma-\Delta$ ADC; b) mathematical model for the ADC mentioned in a); c) 2D extension of the $\Sigma-\Delta$ loop where the linear-transform $s=s_{x} \sin \psi+s_{t}$ is applied; and d) mixed-domain 2D $\Sigma-\Delta$ loop where the bilinear transform $s_{x}=\frac{1-z_{x}^{-1}}{1+z_{x}^{-1}}$ is applied.

3.5 (a) Conventional receiver chain with $N$-bits ADCs, (b) proposed architecture with multiport ADC (1-bit). . . . . . . . . . . . .

3.6 (a) Light cone, RoS and effect of oversampling. (b) Spectrum of the down-sampled signal.

3.7 (a) The inclusion of the proposed 2D spatio-temporal $\Sigma-\Delta$ loop shapes the 2D noise such that the PSD of AWGN is significantly reduced along the line-shaped RoS of the desired RF signal; (b) in the proposed 2D $\Sigma-\Delta$ loop, the distortion components are shaped outside the beam shaped passband of interest. . . . . . . . . . . .

3.8 Conventional $N$-bit ADCs in the array receiver are replaced by spatiotemporal MD $\Sigma-\Delta$ ADCs. These form a multi-port analog-digital mixed-signal control system in which the forward path consists of a $2 \mathrm{D}$-IIR analog beam filter that provides the $2 \mathrm{D}$ version of an ideal integrator $1 / s$. Quantization noise is thus removed from the passband beam that falls on the resonance region $s=0$. A high-resolution beam can then be recovered by digital beamforming; quantization noise is shaped into the side-lobes of the beamformer and is thus attenuated. . . . . . . . . . . . . . . 54

3.9 (a) SFG of a single 2D analog module (2DAM) used to realize the proposed IIR beam filter; (b) spatial interconnection of 2-input-2-output analog modules used to realize the proposed IIR beam filter. . . . . . 56

4.1 (a) Case 1: the prime-axis pencil-beam. (b) Case 2: an off-axis pencilbeam. .................... . . 5

4.2 (a) Parabolic dish antenna, (b) notation used, (c) region of support of parabolic antenna. 
4.3 Spectrum in 2D: (a) wideband with no spatial oversampling, (b) wideband with spatial oversampling $\times 2$, (c) wideband with spatial oversampling $\times 4$; shaped noise for $(\mathrm{d})$ wideband with no spatial oversampling, (e) wideband with spatial oversampling $\times 2$, (f) wideband with spatial oversampling $\times 4 . \ldots \ldots . . \ldots 62$

4.4 SNR improvements: (a) wideband with no spatial oversampling, (b) wideband with spatial oversampling $\times 2,(\mathrm{c})$ wideband with spatial oversampling $\times 4$. . . . . . . . . . . . . . 63

5.1 (a) Positive half (temporal) of the 2D frequency plane showing the finite element pattern; (b) Illustration of the linear transformed spectrum using the transformation matrix $\mathbf{V}$. . . . . . . . . . . . . . . .

5.2 (a) Conventional architecture of an all-digital beamforming array. (b) Overview architecture of the MDLT method, in which linear-transformed spectra are combined to reduce the ADC count. (c) The intact MD spectrum observed by the ULA; linear-transformed spectra for (d) Sub-array 1, and (e) Sub-array 2. . . . . . . . . . . 69

5.3 (a) The spectrum observed by the rectangular aperture, and (b) linear transformed spectrum. Possible linear transform configurations to combine (c) five, and (d) seven channels. . . . . . . . . . . . . . 71

5.4 (a) Uniform rectangular array (URA) with MD linear transforms at RF, and (b) combination stage followed by RF chains (RFCs) and ADCs for a fully digital beamformer. Simulated spectra: (c) observed by the rectangular aperture, and (d) linear-transformed and combined without interference. . . . . . . . . . . . . . . .

6.1 Implementation architectures for (a) TDM-based and (b) CDM-based hardware reduction schemes. . . . . . . . . . . . . 75

6.2 Implementation architecture for a FDM-based hardware reduction scheme. 76

6.3 (a) Digital down-conversion. (b) Poly-phase filterbank. . . . . . . . . . . 82

6.4 Overview of $F_{0}=28 \mathrm{GHz}, 240 \mathrm{MHz} /$ channel four-element FDM array receiver. . . . . . . . . . . . . . . . . .

6.5 (a) Simulation results showing the combined output from the Stage -2 analog down-conversion fed to the ADC. Digital down-conversion and filtering for narrowband signals centered at (b) $125 \mathrm{MHz}$, (c) $375 \mathrm{MHz}$, (d) $625 \mathrm{MHz}$, and (e) $875 \mathrm{MHz}$. (f) Measurements of the combined signal sampled by RFSoC. . . . . . . . . . . . . . . . . . . . . 84

6.6 Experimental setup showing (a) the four-element, 28-GHz receiver antenna array and (b) the transmitter array, and (c) Xilinx RFSoC ZCU1285. 
6.7 Down conversion Stage - 2 setup for 4 element, $28 \mathrm{GHz}$ antenna array. $\quad 86$

6.8 Retrieved carrier signals digitally down-converted to baseband. . . . . . 86

6.9 Theoretical and measured beams at $28 \mathrm{GHz}$ using digital real-time beamforming using a single ADC to sample 4 independent channels.

7.1 Filter-bank responses for (a) the exact 32-point DFT, (b) the 32-point ADFT and (c) absolute error of the ADFT response for the rows with the poorest performance. . . . . . . . . . . . . . . 103

7.2 Log-magnitude error of the frequency response of the rows of the proposed approximations where the least error is bounded to $-60 \mathrm{~dB}$. . 106

7.3 Signal flow graph showing the VLSI architecture to be modified for the proposed architecture based on the selected approximation. Algorithm 1: Replacement of both 32-point DFTs with 32-point ADFT blocks. Algorithm 2: Replacement of only row-wise 32-point DFT with 32-point ADFT blocks leaving column-wise DFT exact. Algorithm 3: Replacement of column-wise 32-point FFT with 32-point ADFT blocks leaving row-wise DFT exact. . . . . . . . . . . . 108

7.4 Schematic diagram of the transpose buffer. . . . . . . . . . . . . 109

7.5 Plots for $\gamma_{k}$, which is used to measure the deviations from orthogonality of (a) Algorithm 1, (b) Algorithm 2, and (c) Algorithm 3 from the ideal case of the 1024-point DFT matrix, with a cap of $40 \mathrm{~dB}$ for visualization purposes. The plots show the output SIR due to spectral leakage among DFT bins due to various levels of approximation. . . 112

7.6 Beamforming architecture of a 1024 ULA receiver using the proposed architecture. The rewiring block performs multiplexing as shown in Fig. 7.3.

7.7 The four worst bins for multi-beam beamforming: (a) exact DFT response, (b) ADFT response, and (c) error for algorithm 1; (d) exact DFT response, (e) ADFT response, and (f) error for algorithm 2; (g) exact DFT response, (h) ADFT response, and (i) error for algorithm

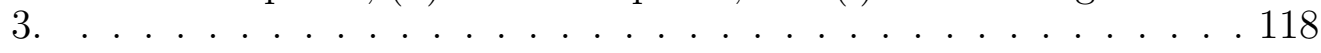




\section{CHAPTER 1 \\ INTRODUCTION}

Advances in radio frequency $(\mathrm{RF})$ technology have led to a high rate of adoption of smartphones and the maturity of corresponding applications. The prolific growth in wireless traffic has enabled zeta bytes of data transmission every year, leading to a requirement for high-speed data communication $[1,2]$. To date, long term evolution (LTE), small cell densification and increased offloading were used to handle the high data demand [3]. Most of the existing cellular communication schemes operate in $700 \mathrm{MHz}$ to $2.4-\mathrm{GHz}$ spectrum, with approximately a $20 \mathrm{MHz}$ channel used by each customer. Since the early 2000s, indoor communication often uses 2.4-GHz and 5-GHz bands for WiFi [4]. The high density of multimedia devices for video and virtual reality applications, multimedia streaming, gaming, wireless docking, and enterprise applications that require high speed and data-intensive connections over wireless local area networks (WLAN) has lead to high data traffic and congestion over indoor networks [5]. Nevertheless, there must be an equal growth of communication bandwidth and signal power to accommodate the exponential growth of wireless capacity [6]. The Shannon capacity theorem [7] states that the capacity $C$ is

$$
C=B \log _{2}(1+\gamma)
$$

where $B$ is the channel bandwidth and $\gamma$ is the signal-to-noise ratio (SNR). Thus, in referencing this equation, it is established that for SNR to remain constant, there must be an exponential growth of bandwidth to enable the growth of capacity. Since lower frequency bands limit the bandwidth availability, it is obvious that communication should happen in a high-frequency spectrum with higher bandwidth to serve the extensive bandwidth requirement. The U.S. Federal communications commission (FCC) has allocated frequency bands between $9 \mathrm{kHz}$ and $275 \mathrm{GHz}$ 


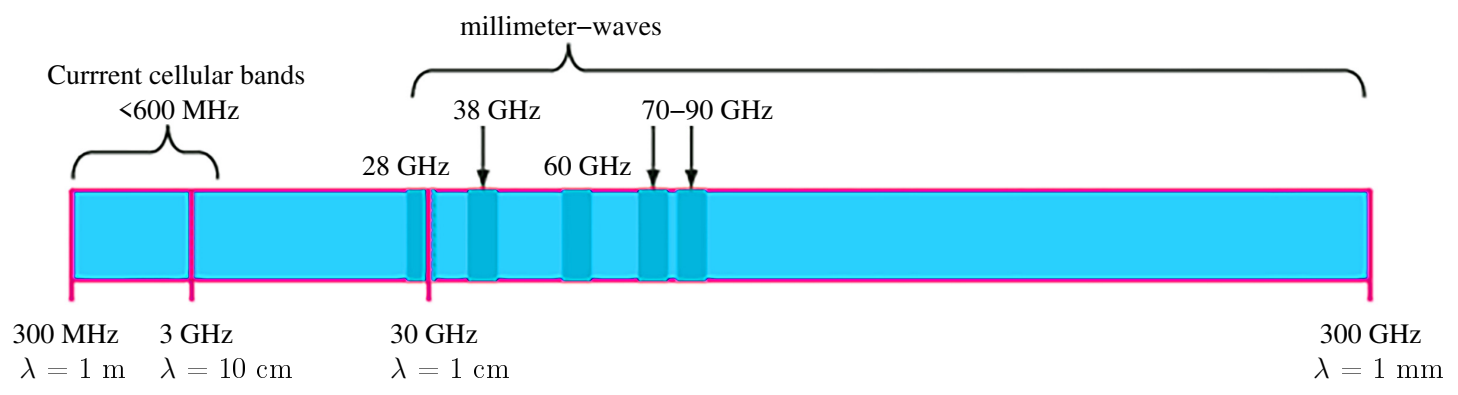

Figure 1.1: The utilization of available radio frequency spectrum.

for use. Since most ground communication uses lower bands, uncongested higher frequency bands are available for use. These higher frequency bands are open for wideband, high-capacity wireless communications, pulse radar, imaging, electronic warfare, pulsar science, and radio astronomy [5, 8].

Striving for higher carrier frequencies obviously reduces the wavelength. The term "millimeter wave" (mmWave) derives from the wavelength of radio signals on frequencies between $30-\mathrm{GHz}$ and $300-\mathrm{GHz}$, which essentially ranges between 1 and 10 millimeters [9]. Due to the use of much higher frequencies in the gigahertz range as carrier frequencies, mmWave communication has a much more abundant spectrum resource as shown in Fig. 1.1. This makes mmWaves quite alluring under the conditions of the intensive spectrum [10].

Currently, the uncongested $60-\mathrm{GHz}$ band is being used for wireless Gigabit Alliance (WiGig), which includes the current IEEE 802.11ad standard and the upcoming IEEE 802.11ay standard [11-15]. This band is unlicensed in the US and other countries with a vast bandwidth $(57-64-\mathrm{GHz})$. Satellite communication uses the 39.5-GHz band and the Wi-Fi standard IEEE 802.11ad operates in the 60-GHz (V-band) spectrum to achieve data transfer rates as high as 7 Gbit/s. However, the increasing demand for spectrum for communication is leading to the use of the 


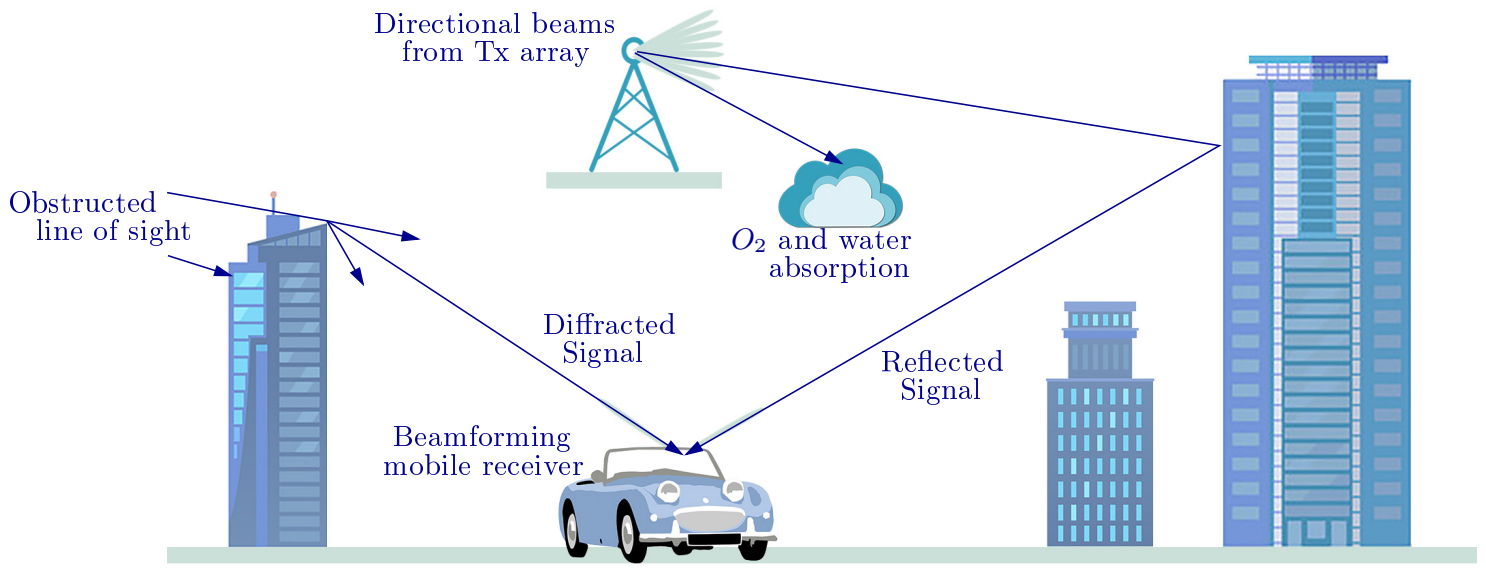

Figure 1.2: Propagation effects of mmWave communication.

previously unused $28-\mathrm{GHz}$ and $73-\mathrm{GHz}$ bands as an extension for fifth-generation (5G) communication.

That being said, frequencies ranging from 100-GHz to $3 \mathrm{THz}$ contain promising bands for next generation $(6 \mathrm{G})$ wireless communication systems due to the vast availability of unexplored spectrum. Undoubtedly, such high frequency communication is associated with a number of implementation issues related to propagation and processing that need to be addressed [16].

\subsection{Challenges Associated with mmWave Communication}

Let us first consider a single element transmission antenna stationed at a distance of $R$ from the receiver to begin with. The Friis free space path loss equation [17] shows the received power as,

$$
\frac{P_{r}}{P_{t}}=\frac{G_{t} G_{r}}{L}\left(\frac{\lambda}{4 \pi R}\right)^{2}
$$


where,

$$
\begin{aligned}
P_{r} & =\text { Received power } \\
P_{t} & =\text { Transmitted power } \\
G_{r} & =\text { Receiver antenna gain } \\
G_{t} & =\text { Transmitter antenna gain } \\
\lambda & =\text { Carrier wavelength } \\
R & =\text { Distance between Tx and } \mathrm{Rx} \text { antenna } \\
L & =\text { Loss factor }(\geq 1) .
\end{aligned}
$$

According to the Friis equation, it is necessary for the communication to happen at a lower frequency (higher $\lambda$ ) to increase the received power. Since this is not an option for mmWave and beyond communication, the only way to increase the received power is by increasing $G_{t}$ and $G_{r}$. However, as the aperture of a lossless, unity gain, isotropic antenna $\left(A_{\text {iso }}\right)$ is $A_{\text {iso }}=\frac{\lambda^{2}}{4 \pi}$, antenna gain has the following relationship with its effective aperture $A_{e}$ and the frequency of operation $[17,18]$.

$$
G=\frac{A_{e}}{A_{\text {iso }}}=\frac{4 \pi A_{e}}{\lambda^{2}}
$$

Equation (1.2) shows that the antenna gain as well as the absolute physical size of an antenna reduces as the frequency of operation increases. On a positive note, this phenomenon makes it possible to pack multiple antennas into a smaller area. Therefore, systems using mmWave and beyond tend to compensate for high path loss by introducing multiple antenna elements without increasing the total physical size of the array $[5,16]$. For example, consider a 2.4-GHz patch antenna would have a wavelength of $\lambda=12.5 \mathrm{~cm}$, which would yield an isotropic effective aperture corresponding to $\lambda^{2} \approx 39 \mathrm{~cm}^{2}$. For a mmWave antenna element at 30 $\mathrm{GHz}$, the wavelength $\lambda=1 \mathrm{~cm}$ and the isotropic effective aperture is proportional 
to $\lambda^{2}=1 \mathrm{~cm}^{2}$. Considering an inter-element spacing of $\frac{\lambda}{2}$, approximately 39 times more antennas at $28-\mathrm{GHz}$ could be accommodated in the same area occupied by a 2.4-GHz antenna array.

Use of multiple antennas introduces spatial sampling to high-frequency RF systems. As a result, terms such as beamforming and multi-dimensional (MD) signal processing are related to mmWave communication. We will discuss this further in the next few chapters.

Diffraction occurs when a wave passes through a slit that is comparable in size to its wavelength. On one hand, at higher frequencies (say 10-GHz and beyond) diffraction is not the dominant propagation mechanism for non-line-of-sight links, but reflection and scattering are. On the other hand, the penetration loss from the propagation through barriers (such as walls) increases with the frequency. Therefore, properly directed, narrow beams are required to reduce path loss in mmWave systems. Figure 1.3 shows that the beam width of a uniform linear array (ULA) narrows as the number of antenna elements increases.

The thermal noise power $N_{0}$ associated with RF communication is defined as,

$$
N_{0}=k T_{c} B
$$

where $k$ is the Boltsman's constant, $T_{c}$ is the temperature and $B$ is the bandwidth. Since mmWave systems have a considerably higher bandwidth than microwave, the high noise bandwidth causes more noise power. To have a better SNR at the receiver, it is required to increase the transmitter gain $G_{T x}$. This can be achieved by using multiple antenna elements and by using constructive interference of their signals at the transmitter to form stronger, highly directional beams. Multiple antenna elements at the receiver can increase the aperture size of the antenna and enable spatial filtering capabilities to effectively apply the gain towards the signal of inter- 


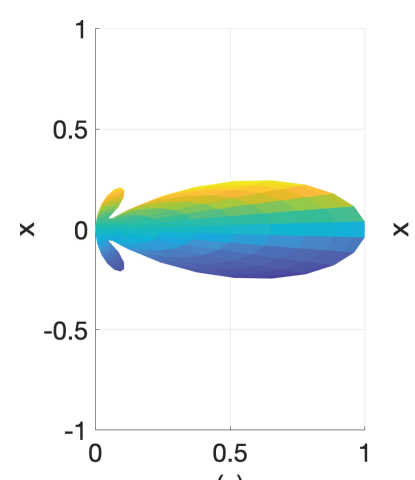

(a)

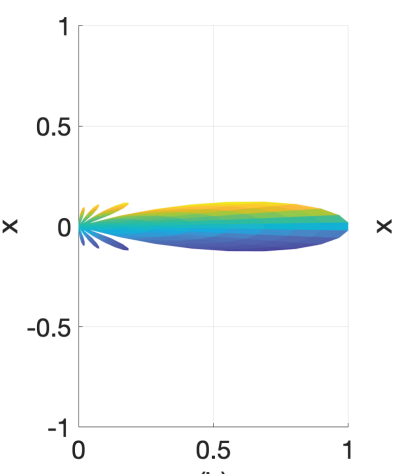

(b)

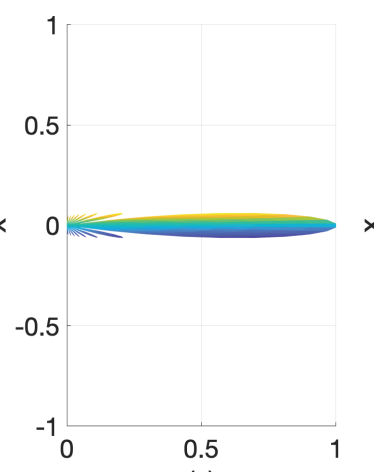

(c)

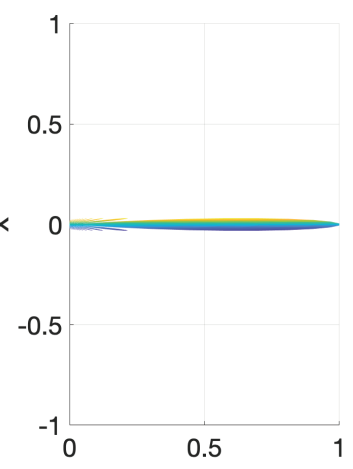

(d)

Figure 1.3: Antenna beams for (a) 4-element, (b) 8-element, (c) 16-element, and (d) 32-element ULAs.

est. MIMO systems with high gain-adaptive beamforming use this phenomenon to increase the coverage and to reduce interference.

In practical realizations of mmWave mobile communication systems, an isotropic radiator or even wide beams allow base stations (BS) to cover multiple user equipment (UE) in a single transmission. However, this is more difficult for narrow beams unless multiple UEs are located in very close proximity to the BS. Although it may seem insurmountable, this problem can be solved by managing beams to cover multiple devices scattered in the space and by using an adaptive control mechanism depending on the situation. Such solutions are often referred to as "beam management," and digital beamforming plays a vital role in this [19].

Owing to the rapid progress in the field of complementary metal-oxide-semiconductor (CMOS) RF integrated circuits, $[20,21]$ beamforming based large-scale mmWave antenna arrays are widely exploited to extend the coverage of mmWave networks $[22,23]$. Interference in mmWave communication can be substantially cut down by using highly directional beams, which renders noise-limited operation rather than interference-limited operation in many cases [24]. 


\subsection{Motivation}

Improvements of wireless applications in the fields of communication, radar, and microwave/ mmWave imaging can be achieved by moving to wideband waveforms such as pulse waveforms and code-division multiple-access waveforms. System bandwidths continue to increase, which makes wideband data conversion at each antenna receiver increasingly challenging - especially for large arrays containing hundreds of elements $[16,25,26]$. There is a push for waveform-agnostic software-defined radios (SDRs) that can operate over a wideband (say $100 \mathrm{MHz}-10-\mathrm{GHz}$ ) using a single RF front-end and a digital signal processing (DSP) back-end. SDRs that are capable of operating over such swaths of bandwidths require analog-to-digital converters (ADCs) and digital-to-analog converters (DACs) that can support sample rates referred to as the Nyquist rate [7] that are greater than twice the desired bandwidth of the waveform for alias-free sampling, which is explained in Nyquist-Shannon sampling theorem. For example, a waveform-agnostic SDR or a radar system operating over $10-\mathrm{GHz}$ of bandwidth requires ADCs/DACs that support sampling at rates greater than $20 \mathrm{GS} / \mathrm{s}$.

Achieving high-precision ADC/DAC circuit realizations has become a challenge with high-speed applications, and a great deal of research has been conducted on the topic $[27,28]$. Despite much progress, the ADC/DAC requirement is always a major bottleneck in wideband systems with a large number of channels/antennas. For example, in order to provide high-capacity channels, an emerging $5 \mathrm{G}$ mmWave wireless system may require base stations that operate on the massive-MIMO principle, which combines traditional beamforming theory with MIMO theory $[29,30]$. These emerging massive MIMO 5G systems would have hundreds (perhaps thousands) of antennas [31-33] operating as antenna arrays. Each of these antenna elements re- 


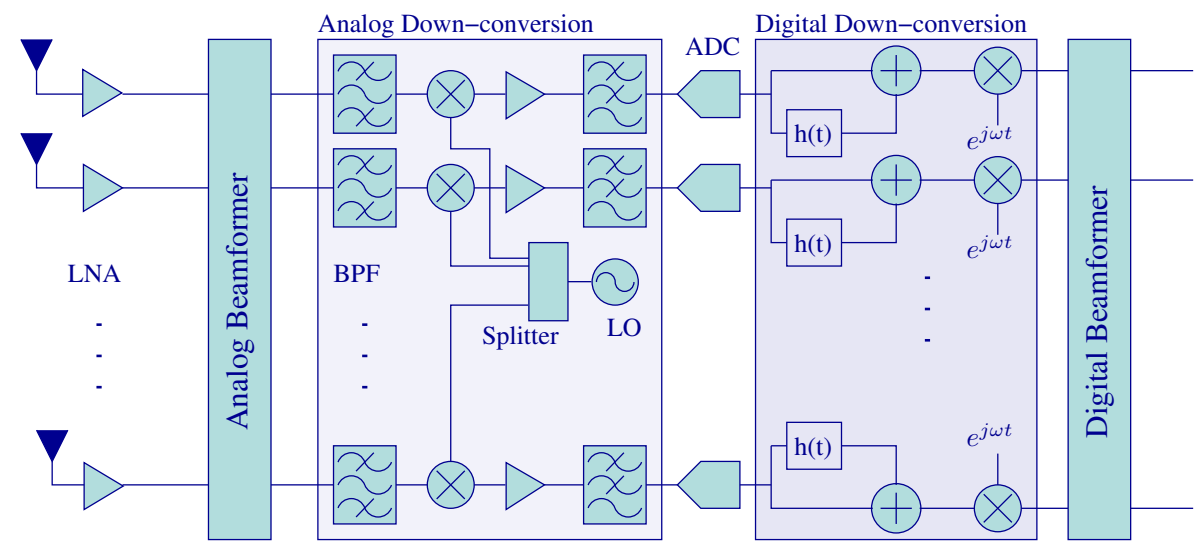

Figure 1.4: Phased array receiver system architecture.

quires a dedicated RF chain that consists of ADC/DAC circuits, LNAs and mixers for processing, as shown in Fig. 1.4. Therefore, for a system of $N$ elements, there would be a requirement for $N$ ADC/DAC circuits.

In certain MIMO applications, antenna arrays are required to process two polarizations (vertical and horizontal) [31-33], which are often referred to as dualpolarized arrays. Needs for dual-polarized arrays exist in wideband radio-astronomy aperture arrays, where scientific experiments require the computation of polarimetric quantities $[34,35]$. Also, military/weather radar systems operating over wide bandwidths require cross-polarized elements that are orthogonal in a spatial plane to receive the horizontal and vertical components of the electric field components [36-38]. In fact, dual-polarized antenna arrays supporting a plethora of elements that are vital for wireless communications, radio astronomy, radar, and microwave imaging [39-45]. The traditional approach of a dedicated data converter for the horizontal and vertical field components necessitate a doubling of the number of ADC/DACs for a given size of antenna array [46,47]. For an $N$-element system with two polarizations, the requirement would be for $2 \mathrm{~N}$ high-speed ADC/DAC circuits at intermediate frequency (IF), and $4 N$ ADC/DACs at baseband. 


\subsection{Contributions and the Outline of the Dissertation}

In most of the systems that achieve RF beams, the C-SWaP is dominated by the ADCs. Therefore, the requirement for a dedicated ADC for every RF chain (each polarization) is a major bottleneck in the reduction of hardware complexity in RF systems with larger bandwidths and higher frequencies.Our research on the reduction of hardware complexity in phased array RF receiver systems focuses two strategies.

1. Reduction of mixed domain/ADC hardware utilization by exploiting MD spectral properties and

2. Reduction of digital hardware complexity (of digital beamformers) using ADFT.

Phased antenna arrays can be identified to have both analog and digital approaches for achieving RF beams [48-53]. Since the use of multiple antenna elements sample the spacial domain, a spatio-temporal analysis of the electromagnetic (EM) characteristics of propagating waves is required to better comprehend the work described in the subsequent chapters. Therefore, Chapter 2 presents a review of MD signal processing, space-time filters, beamforming architectures and MIMO systems.

\subsubsection{Reduction of Mixed Domain/ADC Complexity}

In this research, we followed three different approaches to reduce the ADC complexity in RF receiver systems.

Approach 1: The $\Sigma-\Delta$ architecture is a widely used, well known architecture used in realizing low noise ADCs by shaping the quantization noise outside from the signal bandwidth. A conventional $\Sigma-\Delta$ ADC consists of a oversampling block followed by a feedback loop. The oversampling block reduces the noise power density function (PDF) by spreading the quantization noise over a wider bandwidth. The 
$\Sigma-\Delta$ loop consists of a comparator and an integrator to shape the quantization noise out from the signal bandwidth.

In this approach, the well known $\Sigma-\Delta$ architecture is being explored to reduce the hardware complexity in ADCs while maintaining the effective number of bits (ENOB) by replacing multi-bit quantizer with a single bit quantizer.

In Chapter 3, we investigate the possibility of extending the concept of $\Sigma-\Delta$ to phased array receiver systems by exploiting multi-dimensional signal properties described in chapter 2 in which $N$ - ADCs required in $N$-element phased array systems are replaced by a single multiport ADC. In the conventional approach, a spatio-temporal l extension of $\Sigma-\Delta$ ADCs may use oversampling before the $\Sigma-\Delta$ modulator. Chapter 3, presents a discussion of the possibility of extending the $\Sigma-\Delta$ loop for ULA receiver systems by eliminating the requirement for spatial oversampling. This concept uses the sparsity in the MD frequency domain for a signal with a coherent direction of arrival (DoA).

Focal plane array (FPA) receiver systems that are widely used in satellite and radar communication often consist of rectangular antenna arrays placed at the focal plane of a reflective dish. The MD representation of a received signal for such an array does not have the line spaced sparsity observed in LNAs. The MD spectrum for such a FPA dish is confined to a frustum for a band-limited signal, and the half-angle of the extended cone of the frustum depends on the focal length and the diameter of the dish reflector. Oversampling in the spatial domain can narrow down the frustum-shaped spectrum, which allows the application of $\Sigma-\Delta$ architecture in the MD domain. The idea of extending the $N$-port ADC idea for FPA receivers is discussed in Chapter 4 .

Approach 2: MD spectral properties discussed in Chapter2 (Section 2.5) shows that the region of support (RoS) of a signal captured by a ULA takes the form of a 
line in the spatio-temporal frequency domain. Such sparsity allows the use of spatial multiplexing schemes in the MD frequency domain. Finite element patterns of ULAs constrain the field of view (FoV) for the antenna array, which results in a confined range for the possible RoS. A conventional fully digital beamformer would use a single dedicated ADC per RF channel and would sample the entire MD spectrum without considering the sparsity.

Chapter 5 presents a discussion of the possibility of exploiting sparsity properties and correlations of spatial signals in the MD spatio-temporal spectrum to multiplex multiple RF channels without interference. This approach is explored to divide a ULA into $K$ subarrays and allow the use of a single ADC per sub-array, such that the ADC complexity could be reduced by $\left(1-\frac{1}{K}\right) \times 100 \%$ in the array system.

Approach 3: Conventional communication systems use multiplexing schemes to achieve gains by serving multiple users. A perfect multiplexing scheme may combine two or more streams of information without causing interference and allows the perfect reconstruction of the original information at the destination. Even though traditional approaches have explored multiplexing schemes with the intention of increasing the number of users, the same concept can be extended to achieve reduced hardware resource consumption in phased antenna array systems.

Advanced ADCs used in RF circuits may consume high power and facilitate high data rates. The use of such high-speed ADCs in MIMO applications with relatively narrowband channels may waste the available bandwidths but require more ADCs, as one dedicated ADC is required per channel. Multiplexing methods can be used at phased antenna array receivers to reduce the number of ADC channels required for sampling by combining multiple RF channels. In Chapter 6, we explore the possibility of using a FDM-based approach to save over $75 \%$ of ADC hardware resources in a Xilinx RFSoC ZCU1285 field programmable gate array (FPGA). 


\subsubsection{Reduction of Digital Hardware Complexity}

Beamforming and MIMO systems use digital DFT cores to generate multiple orthogonal beams. The FFT can reduce the computational complexity of an $N$-point DFT from $N^{2}$ to $\mathcal{O}\left(N \log _{2} N\right)$. Since the circuit complexity of a digital design mainly depends on the number of multiplications, it is preferred to have the lowest possible number of digital multipliers. Even though the use of FFT algorithms can reduce the number of multipliers, there exists a lower bound for multiplier complexity (depends on $N$ ), which increases the complexity for large $N$.

However, it is possible to implement an ADFT core to replace the FFT core; this results in an extensive reduction of hardware at a cost of accuracy. However, the loss of accuracy is unlikely to have significant impacts on applications such as digital RF beamforming, where the performance in terms of data rate is dominated by other factors, such as the mutual coupling between elements, mismatches of components, and non-linearities in the RF receiver electronics. In Chapter 7 the digital implementation of a 1024-point ADFT digital core is discussed.

Finally, Chapter 8 summarizes the work and presents the insights that can be gleaned from the conducted research.

\subsection{Scientific Collaborations}

The research on the MD extension of $\Sigma-\Delta$ architecture in developing $N$-port ADCs discussed in Chapter 3 were done in collaboration with Dr. Soumyajit Mandal from University of Florida. His expertise in analog integrated circuit (IC) design lead

to the fabrication of ASICs to implement $N$-port ADCs, and several papers were published in collaboration with the team at the University of Florida. As a part of the research, a method was proposed to eliminate the requirement of spatial 
oversampling in $\Sigma-\Delta$ architecture for sparse signals in the MD spectral domain, and results were presented in IEEE 10th International Workshop on Multidimensional (nD) Systems [28]. This research was extended for focal plane array (FPA) receivers; the results were presented in the 2018 IEEE International Symposium on Circuits and Systems (ISCAS) [27].

Dr. Leonid Belostotski, who is also a specialist in various aspects of RF and mmwave circuits and systems and mixed-signal circuits and who works at the University of Calgary, Canada, collaborated on the work described in Chapters 3 and 5. This collaboration lead to several presentations at number of conferences.

The FDM research work described in Chapter 6 was performed in collaboration with Dr. Theodore S. Rappaport and Dr. Thomas L. Marzetta at NYU Wireless in New York University. Dr. Rappaport is the founding director of NYU Wireless and the author of one of the founding documents on 5G mmWave technology. Dr. Marzetta originated massive MIMO, which is one of the cornerstones of $5 \mathrm{G}$ wireless technology and is the application that is the main focus of this dissertation. Their expertise in the fields of massive MIMO and $5 \mathrm{G}$ mmWave has helped in evaluating the practical relevance of this research. This collaboration has lead to several publications as well as a patent application.

Dr. Renato J. Cintra at Federal University of Pernambuco (UPFE) in Brazil and his student, Dr. Diego Coelho at the University of Calgary, collaborated with us on the ADFT work presented in Chapter 7. Digitally implemented ADFT matrices were derived and provided by Dr. Cintra and his team. Results of this collaboration were published in a DARPA report [54] and an IEEE Access paper [55]. 


\section{CHAPTER 2}

\section{MULTI-DIMENSIONAL SIGNAL PROPERTIES}

This chapter contains a review on the implementation of mmWave MIMO systems, spatial diversity, MD signal processing theories and beamforming. Although mmWave systems have several advantages over microwave systems (4G LTE and before) because of the high carrier frequency, they suffer from certain issues due to the same reason. Table 2.1 summarizes the main differences between microwave communication and mmWave communication in wireless communication systems.

Table 2.1: Comparison of operational characteristics of mmWave and microwave communication systems [56].

\begin{tabular}{lccc}
\hline & Microwave & \multicolumn{2}{c}{ mmWave } \\
\cline { 3 - 4 } WiFi or Cellular & WiFi & 5G Cellular \\
\hline Freq. of operation & $900 \mathrm{MHz}$, & $60 \mathrm{GHz}$ unlicensed & $\begin{array}{c}28 \mathrm{GHz}, \\
38 \mathrm{GHz} \text { or } 71 \mathrm{GHz}\end{array}$ \\
\hline Bandwidth & $1.8 \mathrm{GHz}$ or $2.4 \mathrm{GHz}$ & & $100 \mathrm{MHz}$ to $2 \mathrm{GHz}$ \\
\hline \# Antennas at BS or AP & 1 to 8 & 16 to 32 & 64 to 256 \\
\hline \# Antennas at MS & 1 to 2 & 16 to 32 & 4 to 16 \\
\hline Delay spread & 100 ns to $2 \mu \mathrm{s}$ & 5 ns to $47 \mathrm{~ns}$ & 12 ns to $40 \mathrm{~ns}$ \\
\hline No. of clusters & 4 to 9 & $<4$ & $<4$ \\
\hline Orientation sensitivity & low & medium & high \\
\hline Small-scale fading & Rayleigh & Nakagami & $\begin{array}{c}\text { non-fading } \\
\text { or Nakagami }\end{array}$ \\
\hline Large-scale fading & distant dependent & distant dependent & distant dependent \\
+ shadowing & + shadowing & + blockage \\
\hline Path loss exponent & 2 to 4 & 2 LOS, & 2 LOS, \\
\hline Penetration loss & some & varies & possibly high \\
\hline Channel sparsity & less & more & more \\
\hline Spatial correlation & less & more & more \\
\hline
\end{tabular}




\section{1 mmWave MIMO}

The MIMO communication paradigm-which employs multiple antennas at the transmitter and multiple antennas at the receiver-has been in use for more than two decades. Although extensive research has been conducted on MIMO, different approaches at the transmitter and the receiver are still being explored to achieve higher performance, high data rates and greater reliability by changing the algorithms and system design.

As a result of the vast growth of the frequency of operation, mmWave MIMO systems differ from their low-frequency implementations by the power consumption of ADCs and the hardware complexity of the RF chains. Early mmWave commercial systems IEEE 802.11ad/WiGig and wireless HD have used the analog beamforming model shown in Fig. 2.1 to reduce the RF chain and ADC/DAC complexity. In this approach, the baseband radio at the transmitter sees a single RF chain and a DAC. The analog beamformer takes the same signal and shifts its phases by using configurable time delays to steer the antenna beam to a desired direction [57-59]. In this scheme, called a single-stream MIMO communication, spatial multiplexing gains could not be obtained since it is difficult to extend this approach to multiple streams/multiple users.

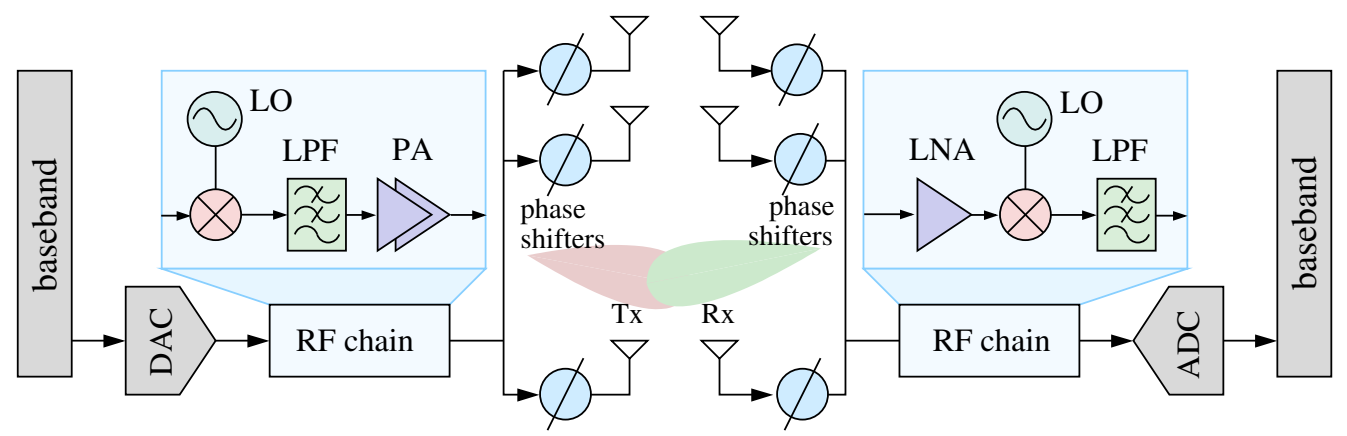

Figure 2.1: Analog beamforming model consists of low noise amplifiers (LNAs), power amplifiers (PAs), low pass filters (LPFs), and local oscillators (LOs) . 


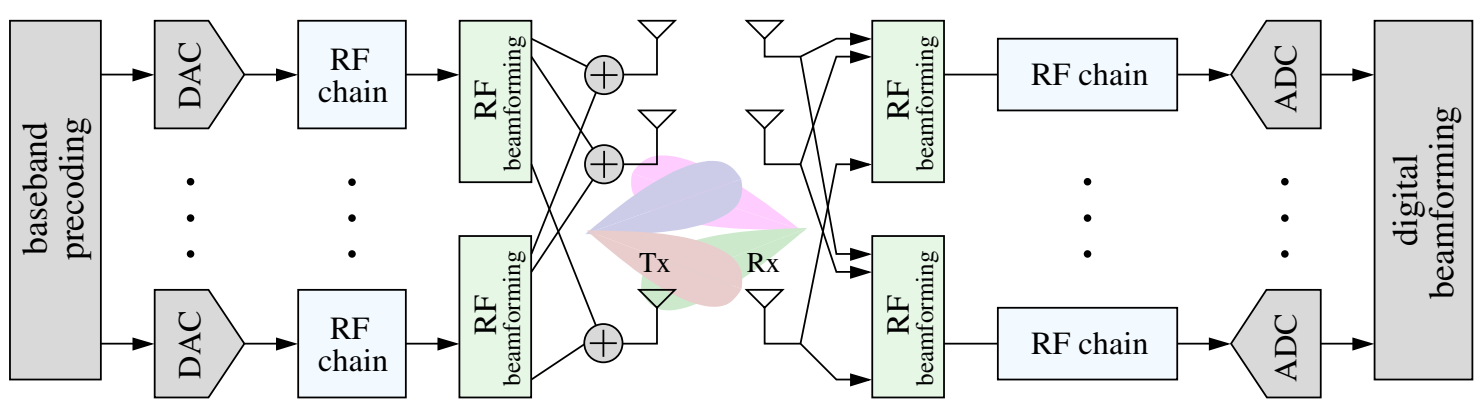

Figure 2.2: Hybrid beamforming model consisting of analog and beamforming components.

This limitation can be successfully overcome by using hybrid analog/digital precoding [60,61], which enables spatial multiplexing and multi-user(MU)-MIMO. Digital beamforming, which can correct for analog limitations, and its implementations are discussed further in the subsequent chapters. Even though digital beamformers provide high flexibility and more degrees of freedom as compared to their analog counterparts, as they require a dedicated RF chain and an ADC for each antenna element. This extensive resource consumption can be handled while also providing similar performance through the implementation of hybrid beamformers, which have both analog and digital components. A typical hybrid beamformer uses RF phaseshifters, true time delays (TTDs) or lenses at the level-1 analog beamforming and uses baseband digital processing at the level-2 digital beamforming. The baseband precoder in a hybrid transmitter side beamformer shown in Fig. 2.2 provides multiple output streams that require a dedicated RF chain and a DAC that is connected to analog beamformers. These analog beamformers could be a set of phase shifters or even a lens that can be used to create multiple physical beams. This process is referred to as second order spatial multiplexing and can be used at the transmitter and/or the receiver to support multiple data streams for a single user or to support multiple users. 
Hybrid beamformers at the receiver could be complex from a wireless point of view, as the channel state information (CSI) that is required to configure the analog beamforming component will not be available until the digital beamformer. However, considering the benefits of hybrid beamforming, this limitation can be handled by using coarse phase shifters at the analog beamformer and using fine tuning in the digital domain [62].

Use of multiple antenna elements in MIMO communication gives rise to three main terms: antenna diversity, spatial multiplexing, and beamforming. The next section discusses antenna diversity from the perspective of wireless technology and how it can be used to facilitate MU communication.

\subsection{Antenna Diversity}

The use of multiple antennas to improve the quality and reliability of a wireless link is known as antenna diversity. Diversity order $L$ is defined as the number of independent communication paths in a multi-antenna system. For comprehension purposes, we will discuss antenna diversity for a receiver and transmitter separately [63].

\subsubsection{Receiver}

For a system with a single-transmitter and two receiver antennas, the received symbols at each of the antenna elements $\left(y_{1}, y_{2}\right)$ for a transmitted symbol $x$ can be written as,

$$
\begin{aligned}
& y_{1}=h_{1} x+w_{1} \text { and, } \\
& y_{2}=h_{2} x+w_{2} .
\end{aligned}
$$


Here, a coherent detection system with known CSI is assumed, where $h_{1}, h_{2}$ are channel coefficients for independent fading and where $w_{1}, w_{2}$ indicate additive white Gaussian noise (AWGN). The vector relationship between the received vector, the channel and the Gaussian noise is given in Eqn. (2.1)

$$
\begin{gathered}
\mathbf{y}=\left[\begin{array}{l}
y_{1} \\
y_{2}
\end{array}\right], \mathbf{h}=\left[\begin{array}{l}
h_{1} \\
h_{2}
\end{array}\right], \mathbf{w}=\left[\begin{array}{l}
w_{1} \\
w_{2}
\end{array}\right] \\
\mathbf{y}=\mathbf{h} x+\mathbf{w}
\end{gathered}
$$

As the receiver is aware of the channel vector $\mathbf{h}$ for a coherent detection, the symbol is detected by following Eqn. (2.2).

$$
\begin{aligned}
\frac{\mathbf{h}^{*}}{\|\mathbf{h}\|} \mathbf{y} & =\frac{\mathbf{h}^{*}}{\|\mathbf{h}\|} \mathbf{h} x+\frac{\mathbf{h}^{*}}{\|\mathbf{h}\|} \mathbf{w} \\
\widetilde{\mathbf{y}} & =\|\mathbf{h}\| x+\mathbf{w},
\end{aligned}
$$

The term $\|h\|$ is the L2 norm of the channel vector, where $\|\mathbf{h}\|^{2}=\mathbf{h}^{*} \mathbf{h}$ and $\mathbf{h}^{*}$ is the conjugate transpose vector of $\mathbf{h}$. This method is often referred tp as maximum-ratio combining (MRC) method. The vector $\tilde{\mathbf{y}}$ is the detected symbol, and $\mathbf{w}^{\prime}$ is the random noise vector normalized by the channel norm. Since the channel norm is a real constant by definition, variables in the normalized noise vector continue to have an independent and identical distribution (IID). The error probability for an MRC at a given SNR can be derived as $P_{e}=Q\left(\sqrt{2\|h\|^{2} S N R}\right)$. Here, the $Q$-function is the tail distribution function of the standard normal distribution [63]. For a given SNR,

$$
\begin{gathered}
\|\mathbf{h}\|^{2}=h_{1}^{2}+h_{2}^{2}+\ldots+h_{L}^{2} \\
\|\mathbf{h}\|^{2} \cdot S N R=L \cdot S N R \cdot \frac{\left(h_{1}^{2}+h_{2}^{2}+\ldots+h_{L}^{2}\right)}{L}
\end{gathered}
$$


Since the mathematical process in this equation is equivalent to averaging out the channel coefficient over multiple independent paths, small scale-fading is negligible for large antenna arrays. This phenomenon has wide applications in massive-MIMO systems and is often referred to as "channel hardening" [64].

\subsubsection{Transmitter}

For a system with two transmitter antennas and a single receiver antenna, transmission by two antennas can either be sent simultaneously or in two different time slots. The spatial diversity order is 1 for simultaneous transmission, as there is only one communication path available when both antennas are transmitting the same symbol. Therefore, the channel variables for two transmitter antennas are added together in order to provide a combined channel variable for the receiver antenna. The received signal for this scenario can be written as

$$
y=\left(h_{1}+h_{2}\right) x+w
$$

Repetitive transmission of the same symbol in two different time slots, as shown in Eqn. (2.6), increases the diversity to an order of 2 because of the availability of two communication paths. However, considering that the antennas will dissipate the same amount of power in both the scenarios, the transmission power observed at the receiver is halved for this case. Another issue associated with this transmission scenario is the reduction of the data rate, as the first antenna is idle in the second time slot and the second antenna is idle in the first time slot.

$$
\begin{aligned}
& \text { slot } 1 \rightarrow y_{1}=h_{1} x+w_{1} \\
& \text { slot } 2 \rightarrow y_{2}=h_{2} x+w_{2}
\end{aligned}
$$


Therefore, simultaneous transmission is required from both the antennas to gain the highest data rate and spatial diversity. A vast amount of research had been carried out in this area under the rubric of space-time coding. The simplest, and yet one of the most elegant, space-time code is widely known as the Alamouti scheme; it is a general code that is used for the transmission to simultaneously achieve spatial and temporal diversity [65]. The Alamouti scheme (defined for two transmitter antennas) achieves this by transmitting two symbols in two adjacent time slots while eliminating idle slots. These antenna diversity techniques can be utilized to improve SNR and to extend the link budget in mmWave MIMO systems.

The majority of $5 \mathrm{G}$ and $6 \mathrm{G}$ applications use MIMO to serve multiple users while maintaining high capacity. To accomplish this, mmWave and sub-THz communication use more than a few antennas and facilitate massive MIMO. Since most of the research work discussed in this dissertation has applications in massive MIMO, the next section introduces the basic concepts of massive MIMO.

\subsection{Massive MIMO}

Massive MIMO can be interpreted as a spatial multiplexing scheme pushed to a wide extreme that is able to serve all users while employing the same time/frequency resources. Essentially a MIMO system has many physically small, low-power antennas to provide aggressive spatial multiplexing. The key point of massive MIMO is that it uses measured channel characteristics as opposed to using assumed channel characteristics. With measured channel information, the beamforming gain can grow linearly with the number of antennas despite the noisiness of the channel [66].

MIMO technology is categorized into three main categories: point-to-point MIMO, MU-MIMO, and massive MIMO. The following sections briefly discuss the first two 


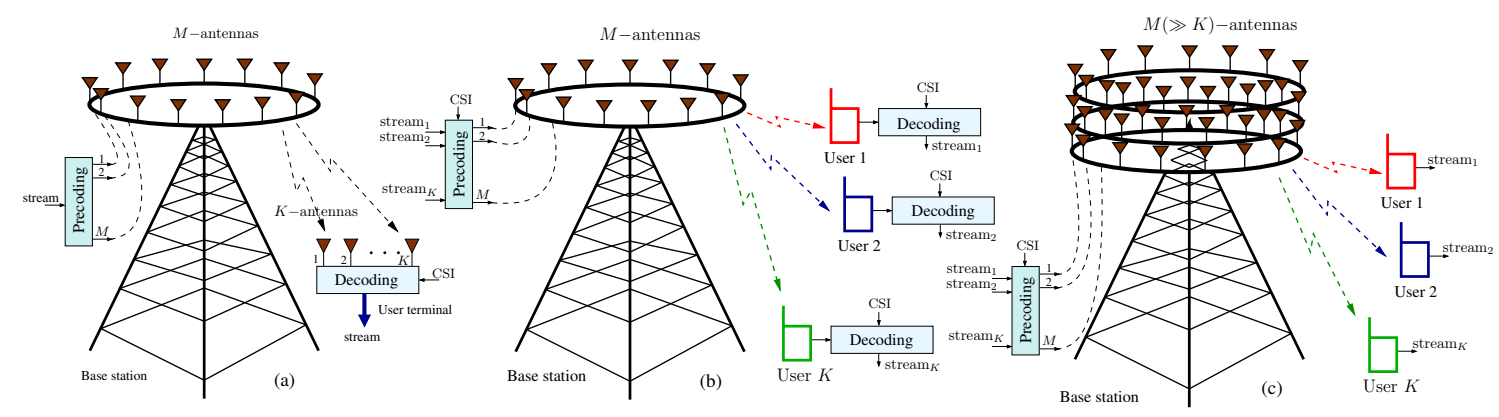

Figure 2.3: Overview of MIMO architecture: (a) point-to-point MIMO, (b) MUMIMO, and (c) massive MIMO.

categories and how they pave the way to massive MIMO. In this discussion, communication links between base stations that are assumed to have $M$ antennas and user equipment having $K$ antennas are considered.

\section{Point-to-Point MIMO}

Point-to-point MIMO is the simplest case of using multiple antennas at the transmitter as well as at the receiver to form a point-to-point communication link by increasing the reliability and capacity of a channel for downlink, as illustrated in Fig. 2.3 (a). In this case, CSI is available at the receiver end for detection purposes.

The time required to train a point-to-point MIMO increases with the system size-consequently, it is not scalable, and multiplexing gains at cell edges are disappointingly low because of high path loss. In addition, line-of-sight (LOS) conditions are stressing for point-to-point MIMO, and the assumption of $\min (M, K)$ independent schemes is practically not the case for compact antenna arrays.

\section{MU-MIMO}

MU-MIMO splits the multi-antenna user into multiple single-antenna users without decreasing the sum-throughput for downlink as shown in Fig. 2.3 (b). For this case, CSI is available at the receiver as well as at the transmitter. Unlike the 
terminals in point-to-point MIMO, user terminals in MU-MIMO require only a single antenna. As long as the transmitter antenna resolution is high enough and the gain is sufficient, use of the single antenna at the receiver does not cause any issues for clear LOS cases, as multiple antennas in the base station can resolve different users and form a beam towards the corresponding receiver.

The uplink and downlink sum spectral efficiencies for MU-MIMO in IID, Rayleigh fading channels are given by Eqn. (2.7) and Eqn. (2.8) respectively,

$$
\begin{aligned}
& c^{u l}= \log _{2}\left|\mathbf{I}_{\mathbf{M}}+\rho_{u l} \mathbf{H H} *\right| \\
& c^{d l}=\max _{\substack{\nu_{k} \geq 0 \\
\sum_{k=1}^{K} \nu_{k} \leq 1}} \log _{2}\left|\mathbf{I}_{\mathbf{M}}+\rho_{d l} \mathbf{H D}_{\nu} \mathbf{H}^{*}\right|
\end{aligned}
$$

where $\nu=\left[\nu_{1}, \ldots, \nu_{K}\right]^{\top}, \rho_{u l}$ is the uplink SNR and $\rho_{d l}$ is the downlink SNR per terminal.

MU-MIMO is more propagation tolerant, but not scalable in its original form and require dirty-paper coding/decoding to cancel the interference [67]. In addition to that, the requirement for channel coherence at both ends of the link is not favorable in economical aspects.

\section{Massive MIMO}

The concept of massive MIMO was proposed by Dr. Thomas L. Marzetta $[68,69]$ and involves adding more base station antennas relative to the number of users (i.e., $M \gg K$ ) for downlink as shown in Fig. 2.3 (c). The analysis described in this chapter is primarily based on Marzetta's book, Fundamentals of Massive MIMO [66]. Underlying Shannon theory for Eqns. (2.7) and (2.8) suggests the optimality of a rough parity between $M$ and $K$ in conventional MU-MIMO, stating that a growth of $M$ would only yield a logarithmic increment in throughput while incurring linear increment of time for training. The massive MIMO concept ignores the dictates 
of Shannon theory by introducing higher $M$ values-and yet, paradoxically, it can achieve better performance than conventional MU-MIMO systems.

In massive MIMO, the CSI is only available to the base station, users do not perform any signal processing, and linear pre-coding/decoding is used instead of dirty-paper decoding. High spectral efficiency is achieved by serving many users simultaneously and using strong directive signals; some efficiency also results from the small amount of interference leakage.

Consider two users communicating over an $M$ element base station with two $M$-dimensional channels denoted by $h_{1}$ and $h_{2}$. If the directions of the channels

are are distinct (i.e., $\frac{h_{1}}{\left\|h_{1}\right\|}$ and $\frac{h_{2}}{\left\|h_{2}\right\|}$ are orthogonal), the base station can fully separate the users in space, as a large $M$ can form multiple beams with high resolution. In the literature, this is referred to as "favorable propagation". It should be noted that, in theory, $M$ has to be infinite to achieve fully orthogonal channels by providing an infinite resolution in the spatial domain. However, fairly orthogonal channels start to appear for approximately $M \geq 50$ in practical scenarios [70].

\subsubsection{Spatial Multiplexing in Massive MIMO}

In order to achieve spatial multiplexing, the same signal is transmitted from all antennas, yet the phase/amplitude is varied per antenna and per subcarrier, depending on the desired user and the channel. In other words, digital beamforming is employed for the $M$-element system. Therefore, massive MIMO implementations require individual control over each of the $M$ antenna elements to form $M$ controllable beams. Since digital beamfomers can form multiple $(M)$ simultaneous beams, it is possible to serve multiple users at the same time achieving spatial multiplexing. 
The information presented in Section 2.1 suggests that mmWave MIMO systems may use hybrid beamforming approaches to form multiple beams and achieve spatial multiplexing. The analog stage in hybrid beamformers reduce its degrees of freedom by combining multiple antenna elements with phase shifters and providing them with the same digital baseband signal. For an example, consider an $M$-element antenna system connected to a hybrid beamformer with $N$-point digital beamforming component where $N<M$. Such a system is capable of forming $N$ simultaneous beams, but phase shifters in the analog component further reduces the control of the individual beams, allowing only one beam to be formed towards a specific direction. The reduction of flexibility in hybrid beamformers is not favorable for massive MIMO applications, which theoretically need infinite, individually controllable beams capable of spanning the entire space. Therefore, digital beamforming is the future of communication, even though hybrid beamformers are being used in certain applications (particularly for vertical beamforming in the first implementations of $5 \mathrm{G})$.

Massive MIMO is based on the fact that the base station is aware of the CSI. The use of an extensive number of antenna elements will result in the formation of highly directional narrow beams. Therefore, the base station should be aware of the direction to which the beam should be aimed.

In a simple, conventional approach, an $M$ element base station may form $M$ angular stationary beams (grid of beams) and index each of the beams. Users can communicate with the base station using time-domain-duplex (TDD) or frequencydomain-duplex (FDD) to separate uplink and downlink, then determine the optimum beam index for communication. However, this approach almost never has a perfect match for the user, as the beam angles are fixed and the approach can result in too much inter-user interference. 
To avoid imperfect beam directions and inter-user interference, massive MIMO uses uplink estimation. In uplink estimation, the user sends a pilot signal, which is detected by using a spatial FFT at the base station. The base station is able to provide a good estimate of the channel based on the measured pilot signal, without considering any other factors as being in LOS or channel nature at the beginning. Uplink estimation is highly scalable, as the channel estimation at the base station happens separately for each of the antennas, regardless of the total number of antennas in the entire system. Since the CSI depends on the frequency, uplink estimation is usable in TDD, which uses the same frequency for uplink and downlink-but is not usable in FDD, which uses different frequencies.

\subsubsection{Downlink Data Transmission}

Information-bearing symbols are combined with measured channel characteristics to create transmitted signals. Using the channel measurements, antennas transmit weighted message-bearing symbols to arrive in-phase at the intended user and outof-phase elsewhere, as shown in Fig. 2.4 (a). Since the channels are orthogonal to each other, a conjugate multiplication can retrieve the information signal; this process is thus known as "conjugate beamforming."

In the uplink-matched filtering shown in Fig. 2.4 (b)-which is the opposite of conjugate beamforming-the base station weights and adds received signals for constructive reinforcement of the transmission from the intended user. This decentralized array architecture follows a simple pre-coding scheme, yet it yields impressive performance. However, zero-forcing may outperform conjugate beamforming-matched filtering for higher signal-to-interference-plus-noise-ratios (SINR). 


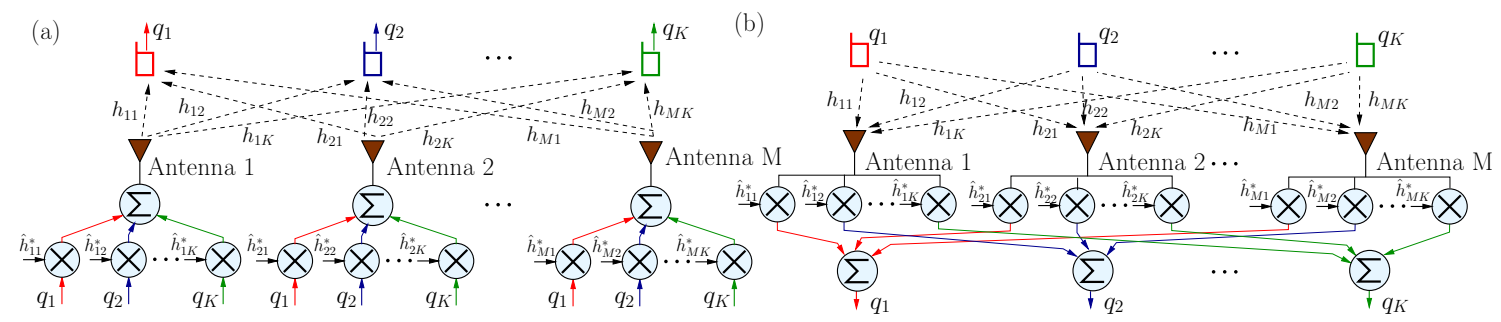

Figure 2.4: (a) Conjugate beamforming for downlink transmission, and (b) matched filtering for uplink transmission in massive MIMO.

\subsubsection{Importance of Beamforming with Measured Channels}

The main characteristic of massive MIMO is replacing open-loop beamforming by channel measurements. As a result, massive MIMO is scalable, and the gain grows linearly with the number of antennas, irrespective of the noisiness of CSI. For assumed channel characteristics, massive MIMO may not be scalable, and the gain would grow logarithmically following Shannon theories in Eqns.(2.7) and (2.8).

As opposed to traditional open-loop beamforming approaches, no tightening of array tolerance is required in using channel measurements rather than using assumed values. In other words, the base station does not have to be aware of the location of the user or the nature of propagation. A traditional open-loop approach would require precise phasing of hundreds or perhaps thousands of antennas in massive MIMO applications, a requirement that is eliminated by using measured CSI.

In a hypothetical scenario, direct measurements could be avoided by using an $M$-point DFT to form $M$ orthogonal beams, and each user could report back the optimum beam for communication. First, the user has to be in LOS with the base station to be able to choose a beam out of $M$ stationary beams for communication. As $M$ increases, phase tolerances for these open loop beams are required to be tighter and more precise to achieve the highest gain. The use of measured CSI can easily avoid these limitations. 

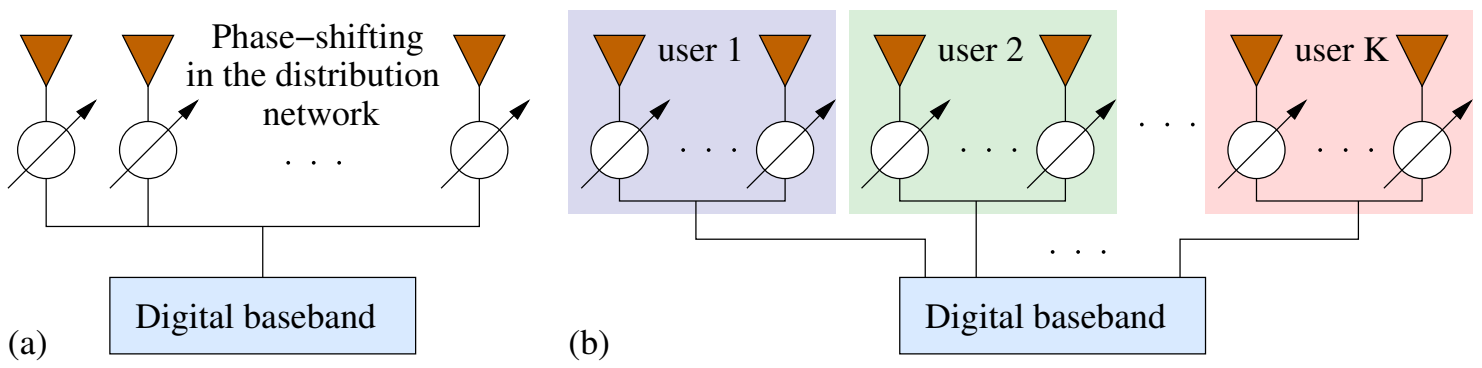

Figure 2.5: (a) Holographic beamformer with PESA, and (b) holographic massive MIMO realization using $K$ PESAs.

\subsubsection{Pilot Contamination}

Each of the mobile users in a massive MIMO system requires an orthogonal pilot. If the same pilot is transmitted by more than one user, the base station may obtain a linear combination of channels. This can happen when the same pilot is being used in adjacent cells in a multi-cellular network, where the base station receives a combination of the pilot from a home cell user and a neighboring cell user. Coherent interference caused by pilot contamination is not eliminated by using more antennas.

Although it is not possible to completely avoid pilot contamination, it is possible to reduce pilot contamination by making the cells smaller and/or using a pilot reuse factor greater than one [66].

\subsubsection{Holographic Beamforming}

Holographic beamforming is a dynamic beamforming technique that uses a softwaredefined antenna (SDA) to employ the lowest C-SWaP [71]. Passive electronically steered antennas (PESAs) are used in realizing holographic beamforming, such that a distribution network that consists of phase shifters (often referred to as "holograms") to form spatial beams. This concept is illustrated in Fig 2.5 (a). The behavior of such a system is similar to a hybrid beamformer, where each digital 
beam is spatially narrowed by an analog stage for better directionality. A PESA has a single RF input followed by a distribution network to vary the directivity of beamforming. According to our discussion regarding Eqns. (2.7) and (2.8), such a single-user beamformer may improve the SINR, yet the capacity has a logarithmic relationship with the number of antennas $M$.

Holographic MIMO may use an array of phase-shifted distribution networks or PESAs, as illustrated in Fig 2.5 (b) to achieve gains from spatial multiplexing in massive MIMO topology. Since narrower beams may spatially multiplex many users with less interference, the capacity of holographic MIMO grows linearly with the number of users. Such a system would behave as a hybrid realization for massive MIMO, where serving $K$ users would require $K$ PESAs [72].

Since the realization of PESA has effective C-SWaP parameters, it may have impressive applications in cost-efficient deployment of massive MIMO. Such an approach may have ULAs or surface realizations for the antenna array. Widely used IID Rayleigh fading channel model may have limitations for such scenarios, as will be discussed in the next section.

\subsubsection{Limitations of Independent Rayleigh Fading Model}

Basic studies in multi-antenna propagation mostly assume IID, Rayleigh fading channels. Spatial diversity studies using Rayleigh fading channels suggest the diver-

sity gain is proportional to the number of antennas and, in characterizing the ergodic capacity for massive MIMO, simple closed-form bounds suggest that the SINR is proportional to the number of antennas [66]. Given that both these conclusions are correct, it is necessary to identify the limitations of the fading model. 


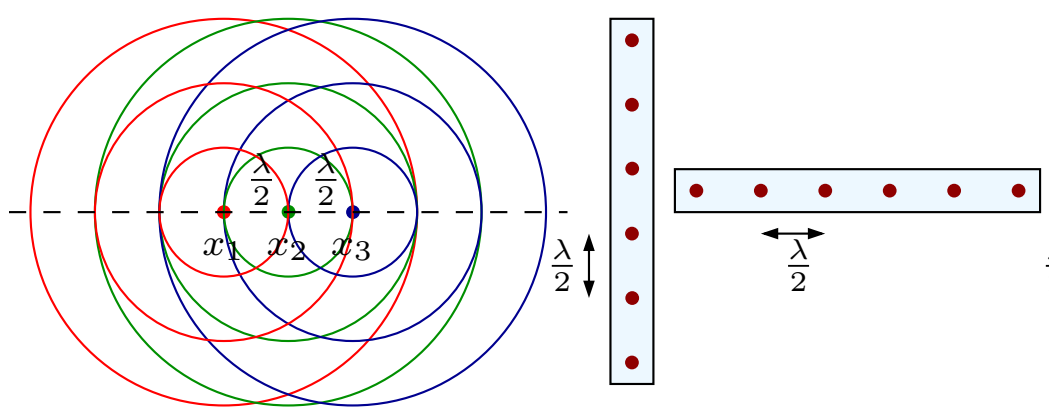

(a)

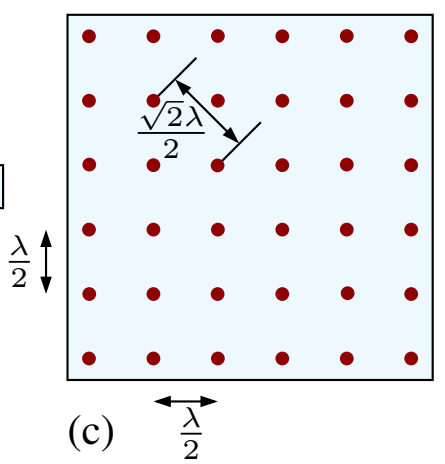

Figure 2.6: (a) Locations for uncorrelation from three antennas in a ULA. (b) ULAs with $\frac{\lambda}{2}$ spacing. (c) A rectangular array with vertical and horizontal spacing of $\frac{\lambda}{2}$.

Consider two points with a spacing of $d$ in the three dimensional (3D) space in an isotropic scattering environment (i.e., an environment where multi-path components are uniformly distributed). The fading model for a propagating signal suggests a correlation of $\operatorname{sinc}(2 d / \lambda)$ between these two points, where $\lambda$ is the wavelength. Therefore, $d$ must be a multiple of $\lambda / 2$ for the two points to be uncorrelated, such that the argument in the sinc function is a non-negative integer. Since IID Rayleigh fading channel coefficients, by definition, follow a Gaussian distribution, any uncorrelated fading will result in independent fading.

The illustration in Fig.2.6 (a), contains circles with radius of multiples of $\lambda / 2$ correspond to independent locations from the corresponding antenna, for a three antenna system. In order to deploy a fourth antenna, while maintaining the Rayleigh fading channel, the antenna must be positioned at a point where all three circles intersect. As illustrated, such a point would only exist in a ULA with an interelement spacing of $\lambda / 2$. This ULA could be placed in any orientation (as shown in Fig. 2.6 (b)) if and only if no other correlating sources exist in the 3D space. Therefore, the IID Rayleigh fading model cannot be applied for a rectangular array shown in Fig. 2.6 (c), where the inter-element spacing along the diagonals is no longer $\lambda / 2$ [73]. 
Large intelligent surfaces (LIS) and reconfigurable intelligent surfaces (RIS) are gaining the attention of researchers in antenna array systems. As the term "surface" in their names suggest, LIS and RIS are planar arrays and, therefore, an IID fading model cannot be applied. For such systems, a more general spatially correlated Rayleigh model can be applied [74].

\subsection{EM Characteristics of Planar Wave Propagation}

Spatial multiplexing and beamforming concepts can be combined to improve channel performance. Subsequent chapters discuss the hardware complexity reduction and beamforming for mmWave MIMO systems from the perspective of MD signal processing. This section contains a detailed review on spatio-temporal signal processing concepts that provide the basis for a better understanding of the processes discussed in later chapters.

MD signal processing considers the spatial dimension along with the temporal dimension. Multiple antenna elements used in phased antenna arrays are used to sample a signal in the spatial domain, such that discrete transforms (such as Fourier and $\mathrm{Z}$ transforms) are applicable over the spatial dimension. In other words, temporal transfer functions can be extended to take spatial dimensions into account.

The far-field for an EM wave $x(t)$ emitted by an isotropic transmitter (shown in Fig. 2.7), exists beyond a distance $d$ from the transmitter for $d>\frac{2 D^{2}}{\lambda}$. Here, $\lambda$ is the wavelength and $D$ is the aperture of the transmitter antenna [75]. A far-field MD planar wave $w(t)$ defined in $t=\left(t_{1}, t_{2}, \ldots, t_{n}\right) \in \mathbb{R}^{n}$ is called a planar wave if there exists an MD vector $\mathbf{d}$ such that $w(t)$ is constant valued everywhere in each of the planes $\mathbf{d}^{T} t=l ; \forall l \in \mathbb{R}$. 


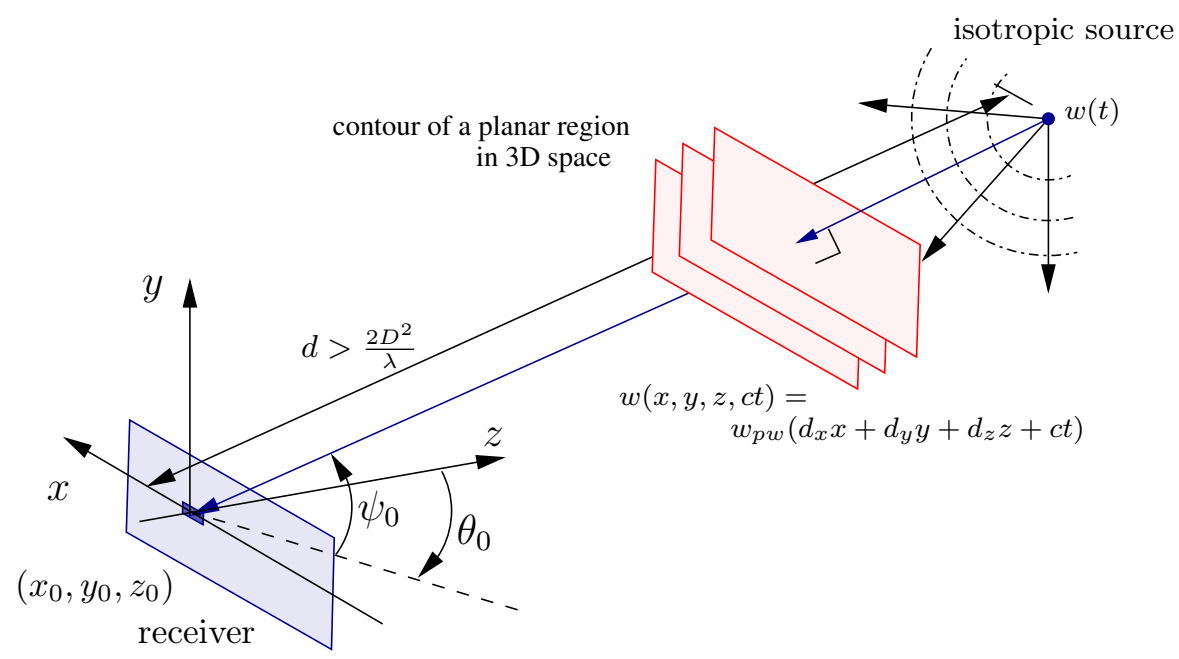

Figure 2.7: Propagating wave in the 3D space with a DoA of $(\psi, \theta)$.

Consider either the transverse electric $E(x, y, z, c t)$ or magnetic $H(x, y, z, c t)$ field of a propagating electromagnetic planar wave, where $(x, y, z) \in \mathbb{R}^{3}$ is the $3 \mathrm{D}$ space and $t \in \mathbb{R}$ is the time-continuous domain where $c \approx 3 \times 10^{8} \mathrm{~ms}^{-1}$ is the speed of light. A hyper plane can be defined in the given 4D continuous spatio-temporal domain as,

$$
d_{x} x+d_{y} y+d_{z} z+c t= \pm \lambda
$$

where $\left(d_{x}, d_{y}, d_{z}\right) \in \mathbb{R}$ and $d_{x}^{2}+d_{y}^{2}+d_{z}^{2}=1$. The hyper plane $\lambda$ defined here is merely a parameter and is not related to the wavelength of the propagating signal. The DoA of the $w_{p w}$ signal in the $3 \mathrm{D}$ space is denoted by the unit vector $\hat{\mathbf{d}}$, where $\hat{\mathbf{d}}=\left[\begin{array}{lll}d_{x} & d_{y} & d_{z}\end{array}\right]^{\top}$.

Therefore, the one-dimensional (1D) function of a wave propagating in this $4 \mathrm{D}$ hyper-plane is defined as,

$$
w(x, y, z, c t)=w_{P W}(\lambda) ; \forall \lambda \in \mathbb{R}
$$

Equation (2.10) shows that there exists a corresponding iso-surface in $(x, y, z, c t)$ for every value of the parameter $\lambda$. 
Figure 2.7 illustrates a traveling planar wave $w_{P W}(x, y, z, c t)$ in the $4 \mathrm{D}$ spatiotemporal domain at an arbitrary time and located at the far-field of the isotropic transmitter for the iso-surface $\lambda$. Therefore, we can conclude that there exists a set of infinite iso-surfaces (described in Eqn. (2.10)) in 4D, over time $t$ with speed $c$ for a propagating signal. This signal could be either temporally narrowband or wide band, depending on the 1D spectral properties of the $c$-scaled temporal signal $w(c t)$.

With reference to the notation in Fig. 2.7, the polar form of the $4 \mathrm{D}$ parametric $\lambda$ can be expressed in terms of the azimuth $\theta \in[0, \pi]$ and the elevation $\psi \in[0,2 \pi]$ as,

$$
\lambda=-x \sin \theta \cos \psi+y \sin \psi+z \cos \theta \cos \psi+c t
$$

and the same polar coordinate system can be used to redefine the unit vector $\hat{\mathbf{d}}$ as,

$$
\hat{\mathbf{d}}=\left[\begin{array}{lll}
d_{x} & d_{y} & d_{z}
\end{array}\right]^{\top}=\left[\begin{array}{lll}
-\sin \theta \cos \psi & \sin \psi & \cos \theta \cos \psi
\end{array}\right]^{\top}
$$

For convenience, this discussion is limited to propagating waves with their electric or/and magnetic field on planar surfaces (3D) and straight lines (2D). The $z$ coordinate is zero for a rectangular array receiver system; thus the parametric relationship between $(x, y, z)$ in Eqn. (2.11) reduces to,

$$
\lambda_{3 D}=-x \sin \theta \cos \psi+y \sin \psi+c t
$$

Next, the planar region for a 3D spatio-temporal planar wave can be expressed as,

$$
\begin{aligned}
p w_{3 D} & \left.=w(x, y, c t)=w\left(d_{x} x+d_{y} y+c t\right)\right) \\
& =w(-x \sin \theta \cos \psi+y \sin \psi+c t)
\end{aligned}
$$

Similarly, Eqn. (2.11) can be reduced for the case of a ULA placed on the $y$ axis in Fig. 2.7, (i.e., $\left.p w_{2 D}=w(0, y, 0, c t)\right)$ by disregarding the $x$ and $z$ components as,

$$
\lambda_{2 D}=y \sin \psi+c t
$$




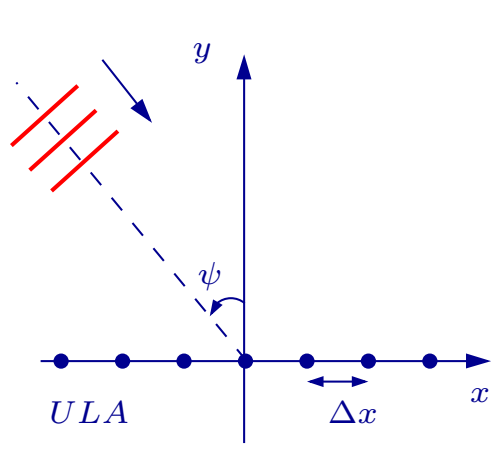

(a)

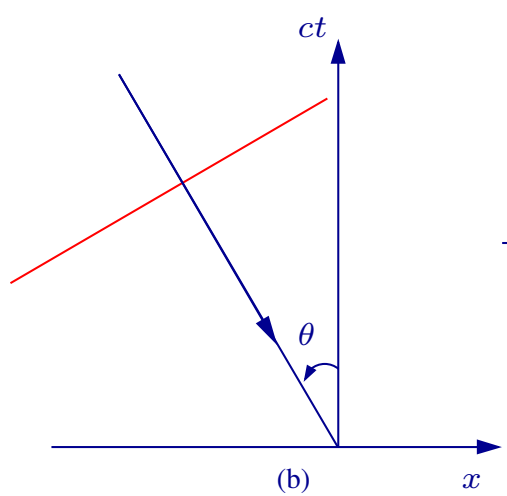

(b)

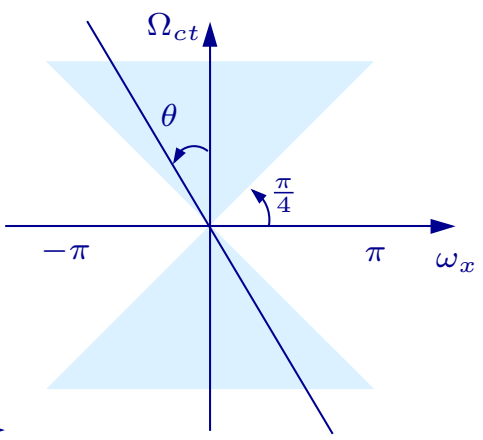

(c)

Figure 2.8: (a) Spatial domain, (b) 2D spatio-temporal, and (c) 2D continuoustime-discrete-space frequency domain illustration of a planar wave impinging on a ULA.

For such a ULA receiver system, the Eqn. (2.9) will be reduced to $d_{y} y+c t= \pm \lambda$. Consider a ULA placed on the $x$-axis by swapping the $x$-axis and the $y$-axis for the convenience, in which the DoA is measured with respect to the $y$-axis. Figure 2.8 (a) shows such a system, where a planar wave is arriving at an angle of $\psi$ measured with respect to the $y$-axis. For this system, the parametric relationship in Eqn. (2.15) can be rewritten with respect to $\psi$ as $\left(-\sin \psi_{0}\right) x+c t=\lambda$. If the DoA in the space-time domain is $\theta^{1}$ where,

$$
\theta=\tan ^{-1}(\sin \psi) .
$$

As illustrated in Fig. 2.8, the DoA is constrained to $[-\pi / 2, \pi / 2]$. Therefore, the angle $\theta$ is confined in the region in Fig. 2.8 (c) where $-\frac{\pi}{4} \leq \theta \leq \frac{\pi}{4}$.

This property is used in practice to analyze the operation of phased array systems. In order to begin with the MD signal properties of planar waves, let us consider a ULA where the spatial sampling distance is $\Delta x$.

\footnotetext{
${ }^{1}$ The angle $\theta$ is merely an angle in the space-time domain and does not depend on the azimuth angle $\theta$.
} 


\subsection{Properties of ULAs and Rectangular Arrays}

In most practical implementations of mmWave MIMO systems, 2D or 3D planar waves are sampled in the spatial domain using ULAs or rectangular arrays. Because of the finite aperture size of antenna elements, the spatial dimension is discretized and the temporal dimension could either be continuous or discrete based on the signal processing domain. This section contains a discussion of planar wave characteristics, starting from the continuous domain and then extends to the discrete domain.

To begin our discussion of the spectral properties of planar waves, let us first consider the continuous-time Fourier transform (CTFT) of the planar wave equation (Eqn. (2.10)), which can be expressed as Eqn. (2.17).

$$
P W_{4 D}\left(\Omega_{x}, \Omega_{y}, \Omega_{z}, \Omega_{c t}\right) \equiv \iint_{x, y, z, c t=-\infty}^{+\infty} \iint_{4 D} p w_{4 D}(x, y, z, c t) e^{-j\left(\Omega_{x} x+\Omega_{y} y+\Omega_{z} z+\Omega_{c t} c t\right)} d x d y d z d c t
$$

where $\left(\Omega_{x} x, \Omega_{y} y, \Omega_{z} z, \Omega_{c t} c t\right) \in \mathbb{R}^{4}$. The angular frequencies are defined as $\Omega_{k}=$ $2 \pi f_{k}$, where $k \in\{x, y, z, c t\}$. Note that the temporal frequency is scaled by $c$ such that $f_{c t}=f_{t} / c$. Based on the analysis in [76], Eqn. (2.17) can be simplified to have the form of,

$$
P W_{4 D}\left(\Omega_{x}, \Omega_{y}, \Omega_{z}, \Omega_{c t}\right)=W\left(\Omega_{t}\right) c \Omega_{c t} \cdot \delta\left(d_{x} \Omega_{c t}-\Omega_{x}\right) \cdot \delta\left(d_{y} \Omega_{c t}-\Omega_{y}\right) \cdot \delta\left(d_{z} \Omega_{c t}-\Omega_{z}\right)
$$

where $W\left(\Omega_{t}\right)$ is the CTFT of the $1 \mathrm{D}$ temporal signal $w(t)$ with the relationship

$w(t) \stackrel{\mathcal{F}}{\Longleftrightarrow} W\left(\Omega_{t}\right)$ and $\delta($.$) is the impulse function. Eqn. (2.18) can be simplified$ for the $2 \mathrm{D}$ and $3 \mathrm{D}$ cases by simply eliminating the corresponding impulse terms $\delta\left(d_{y} \Omega_{c t}-\Omega_{y}\right)$ and/or $\delta\left(d_{z} \Omega_{c t}-\Omega_{z}\right)$ respectively.

Even though the analysis is performed for a continuous space domain, finite aperture sizes of antenna elements break the continuity of the spatial domain. This 

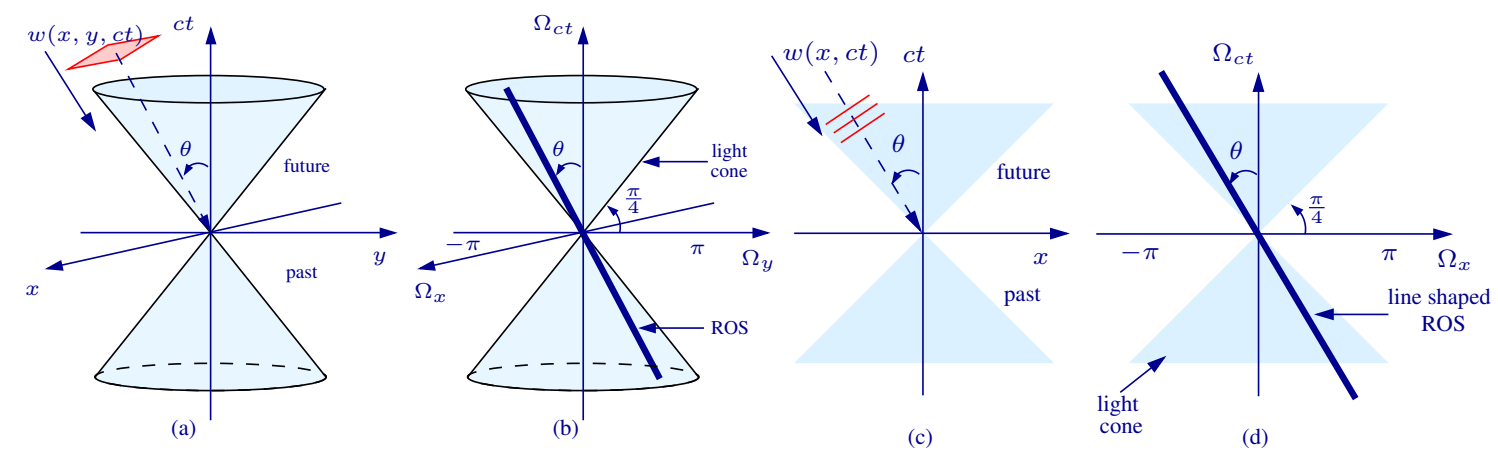

Figure 2.9: (a) The spatio-temporal illustration of a 3D planar wave impinging on a planar surface $(z=0)$ and $(b)$ the $3 \mathrm{D}$ frequency domain representation. (c) The spatio-temporal illustration of a $2 \mathrm{D}$ planar wave impinging on a line $(y=0, z=0)$ and $(\mathrm{d})$ the 2D frequency domain representation.

discussion considers uniformly spaced rectangular and linear antenna arrays, as these are the most widely used in RF applications [77]. However, it should be noted that non-uniform geometries and other sampling grids such as triangular [78], circular [79], and hexagonal [80] are being used in certain applications.

To begin with the analysis for sampled spatial domain, an infinite grid is assumed. A spatially sampled, yet temporally continuous signal can be expressed as $w_{D S C T, 3 D}\left(n_{x}, n_{y}, c t\right)$, where $\left(n_{x}, n_{y}\right)$ are the spatial index of the grid. The frequency domain representation for such a signal can be derived using Eqn. (2.17) as follows:

$W_{D S C T, 3 D}\left(\omega_{x}, \omega_{y}, \Omega_{c t}\right)=\int_{c t=-\infty}^{+\infty} \sum_{n_{x}=-\infty}^{+\infty} \sum_{n_{y}=-\infty}^{+\infty} w_{D S C T, 3 D}\left(n_{x}, n_{y}, c t\right) e^{-j\left(\omega_{x} n_{x}+\omega_{y} n_{y}+\Omega_{c t} c t\right)} d c t$.

Following [76], Eqn. 2.19 can be simplified as,

$$
W_{D S C T, 3 D}\left(\omega_{x}, \omega_{y}, \Omega_{c t}\right)=\frac{1}{\Delta x \Delta y} \sum_{n_{x}=-\infty}^{+\infty} \sum_{n_{y}=-\infty}^{+\infty} P W_{3 D}\left(\frac{\omega_{x}-2 \pi n_{x}}{\Delta x}, \frac{\omega_{y}-2 \pi n_{y}}{\Delta y}, \Omega_{c t}\right)
$$

where $\omega_{x}=\Omega_{x} \Delta x$, and $\omega_{y}=\Omega_{y} \Delta y$ in which $\Delta x, \Delta y$ are the inter-element spacing in the $x$-axis and $y$ - axis, respectively. The planar wave function $P W_{3 D}($.$) is the 3 \mathrm{D}$ CTFT function derived from Eqn. (2.17). Similar to the spectral repetition in the 
temporal Fourier transform, the spatially sampled signal spectrum repeats infinitely with a period of $2 \pi$ along both $\omega_{x}$ and $\omega_{y}$. Therefore, according to the Nyquist theorem, we must keep $\left(\omega_{x}, \omega_{y}\right) \leq \pi$ to avoid spatial aliasing. For temporally bandlimited signals (i.e., where $\Omega_{c t} \leq c^{-1} \Omega_{c t, \max }$ ), and the spatial bandwidth $\left(\Omega_{x}, \Omega_{y}\right)$ is greater or equal to $c^{-1} \Omega_{c t, \max } \tan \theta_{\max }$ (refer to Fig. $2.9(\mathrm{~b})$ ). Since $\tan \theta=\sin \psi$, the sampling criterion to avoid spatial aliasing can be expressed as,

$$
(\Delta x, \Delta y) \leq \frac{c}{2 \cdot f_{t, \max } \cdot \sin \left(\psi_{\max }\right)}
$$

For an omni-directional antenna, $\psi_{\max }=\frac{\pi}{2}$. Eqn. (2.21) is simplified to,

$$
\begin{aligned}
& (\Delta x, \Delta y) \leq \frac{c}{2 f_{t, \max }} \text { or } \\
& (\Delta x, \Delta y) \leq \frac{\lambda_{\text {min }}}{2}
\end{aligned}
$$

where $\lambda_{\min }$ is the wavelength corresponding to the highest frequency of interest $f_{\max }$.

A simpler 2D spectral analysis may consider a wide band, planar wave arriving at a ULA with a DoA of $\psi$, where $\left(-\frac{\pi}{2} \leq \psi \leq \frac{\pi}{2}\right)$, as discussed with regard to Fig. 2.8 (a). The spatial domain is sampled by a finite number of finite aperture antenna elements uniformly spaced at $\Delta x$. The DoA $\psi$ is measured with regard to the broadside direction of ULA, in a counter-clockwise direction as illustrated. The signal received by each of the elements in the ULA produces a discrete-in-space and continuous-in-time signal that can be written as,

$$
w_{D S C T}\left(n_{x}, c t\right)=w_{s}\left(-n_{x} \Delta x \sin \psi+c t\right) .
$$

Two adjacent antenna elements have a time shift of

$$
\Delta T_{D}=\frac{\Delta x \sin \psi}{c}
$$


where the element at the left has a time advancement of $T_{D}$ for $0 \leq \psi \leq \pi / 2$ in the received waveform. ADCs with sampling frequencies of $f_{s}=1 /(\Delta T)$ are used at each antenna element to discretize the continuous time signal, where $f_{s}$ is chosen to satisfy the temporal Nyquist criterion. The temporally discretized signal can be expressed as,

$$
w_{D S D T}\left(n_{x}, n_{c t}\right)=w_{s}\left(-n_{x} \Delta x \sin \psi+c n_{c t} \Delta T\right)
$$

\subsubsection{Region of Support}

The region of support (RoS) is defined as the region where the magnitude of the frequency spectrum is not defined to be zero. Equation (2.18) suggests that the RoS of the 4D spectrum is confined by the intersection of $4 \mathrm{D}$ hyper-planes that are defined by each of the $\delta$ functions. (i.e., $d_{x} \Omega_{c t}-\Omega_{x}=0, d_{y} \Omega_{c t}-\Omega_{y}=0$ and, $\left.d_{z} \Omega_{c t}-\Omega_{z}=0\right)$. For the sake of simplicity and to indicate the relevance of the discussed research work, this chapter is focused on 2D and 3D planar waves.

For a planar wave impinging on a surface in the $3 \mathrm{D}$ space $(Z=0)$ shown in Fig. 2.9 (a), the RoS is confined to a line in the $3 \mathrm{D}$ spectrum. The range of $\theta$ is constrained by the spatial DoA such that $-\frac{\pi}{4} \geq \theta \geq \frac{p i}{4}$, as presented in Eqn. (2.16).

In the $2 \mathrm{D}$ spatio-temporal frequency domain, the RoS of $W\left(\Omega_{x}, \Omega_{c t}\right)$ is confined to a straight line passing through the origin, which creates an angle of $\theta$ as measured from $\Omega_{c t}$ axis, where $\tan \theta=\sin \psi$ as shown in Fig. $2.9(\mathrm{c}-\mathrm{d})$. Here, $\left(\Omega_{x}, \Omega_{c t}\right) \in \mathbb{R}^{2}$ is the domain of the continuous $2 \mathrm{D}$ frequency spectrum, where $\Omega_{c t}$ represents the normalized temporal frequency. 


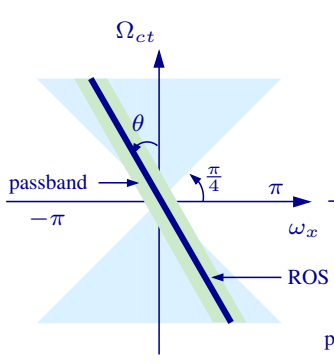

(a)

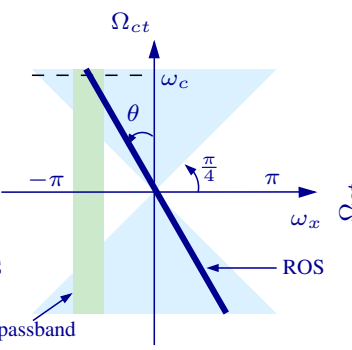

(b)
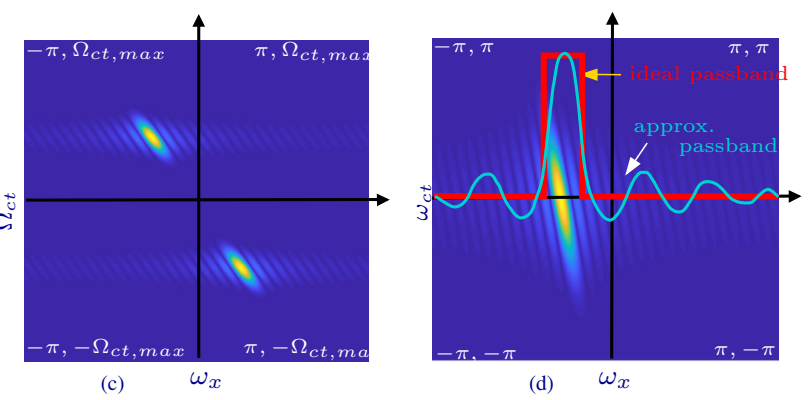

Figure 2.10: MD filtering for a (a) wideband (b) narrowband ULA. Received MD spectrum for a ULA in (c) RF and (d) downsampled at baseband.

\subsubsection{Practical Aspects}

In practice, antennas have a directional receive pattern, such that the main lobe extends in the broadside direction and is directional in nature, where the radiation pattern is reduced to a pre-determined level (say $-10 \mathrm{~dB}$, or $90 \%$ power) at angles $\psi=\psi_{\max }$. An example of such an antenna pattern takes the shape of $\cos ^{n}(\psi)$.

Since $-1 \leq \sin \psi \leq 1$, the range of $\theta$ is constrained by $-1 \leq \tan \theta \leq 1$ (i.e., $-\frac{\pi}{4} \leq \theta \leq \frac{\pi}{4}$ ). When directional antennas that do not produce signals beyond $\psi_{\max }$ are used in the array in place of omni-directional elements, the spectral RoS is further constrained to line-shaped regions that are within the dual-fan bound by $\theta_{\max }= \pm \tan ^{-1}\left(\sin \psi_{\max }\right)$ [81-83]. This phenomenon and its applications are discussed in greater detail in Chapter 5.

\subsection{Spatio-temporal Filter Implementations}

The received signal spectrum for a planar wave lies on a line passing through the origin in the MD spectral domain, as discussed in Section 2.5.1. Therefore, spacetime filters that are implemented to achieve selective enhancements are required to have the passband aligned with the line-shaped RoS. This is illustrated in Fig. 2.10 for the scenario where a 2D spatio-temporal planar wave is captured by a ULA. 
For a narrowband signal, the passband of the filter does not essentially lie on the RoS line, but it may cover the considered narrowband frequency of operation as shown in Fig. 2.10 (b). Such filters are applicable in most of the narrowband, analog phase-shifting-based receiver arrays to achieve directional enhancements. However, these passbands are known to suffer from a phenomenon widely known as beam squinting, which leads to a deviation in the beam direction for not-so-highlynarrowband systems $[50,84]$. The ideal passband filter that can be realized in the digital or analog domain for a temporally wideband signal should have the form shown in Fig. 2.10.

The concept of beamforming discussed in Section 2.1 can also be explained in the form of spatial filtering at the receiver side, where the signal of interest arriving from a given DoA is subjected to selective enhancements. The MD spectrum of such beamforming filters have the same forms shown in Fig. 2.10, which are based on their bandwidths. Similar to any other MD filter, beamforming can also be achieved either in IF or in baseband according to the design specification. However, most of the existing digital beamformers operate at low-IF frequencies or in the baseband in order to reduce the hardware complexity. Downconversion of a signal $w(w, c t) \stackrel{\mathcal{F}}{\Longleftrightarrow} W\left(\omega_{x}, \omega_{c t}\right)$ has the modulation property of the MD Fourier transform given by,

$$
w(w, c t) e^{-j \omega_{0} c t} \stackrel{\mathcal{F}}{\Longleftrightarrow} W\left(\omega_{x},\left[\omega_{c t}+\omega_{0}\right]\right)
$$

Figure 2.10 (c) shows the narrowband RF signal spectra of a 2D planar wave signal with a DoA of $(\pi / 3)$, and Fig. 2.10 shows the baseband spectrum for the same signal where the downconverion is followed by downsampling. Even though the ideal passband expected for such an MD signal has a rectangular shape, practical implementations often follow an approximation. An application of such spatial filters 
is discussed in Chapter 5 .

\subsection{Digital Beamforming}

While digital beamforming has high flexibility, reconfigurability and higher degrees of freedom as compared to analog beamforming, it requires one ADC per RF chain (or two per RF chain for baseband I-Q components). Section 2.1 discussed the limitations of analog beamforming and the necessity for hybrid beamforming, which contains both analog and digital beamforming components to achieve the best of both worlds. This section discusses digital beamforming in greater detail as well as strategies for its implementation.

Figure 2.11 (a) shows the general model for a $N$-element phased antenna array with Nyquist spacing i.e., $\Delta x=\lambda_{\min } / 2$, where $\lambda_{\min }$ is the wavelength corresponding to the highest frequency of interest. Consider a 2D spatio-temporal planar wave $w_{m}\left(n_{x}, t\right)$ arriving at the considered ULA at a DoA of $\psi$. The continuous time output vector of the array caused by the impinging signal is defined as $\mathbf{x} \in \mathbb{C}^{(N, 1)}$, where $\mathbf{x}=\left[\begin{array}{llll}x_{o}(t) & x_{1}(t) & \ldots & x_{N-1}(t)\end{array}\right]^{\top}$ and $x_{n_{x}}(t)=w_{m}\left(n_{x}, t\right)$. The mixed domain output vector $\mathbf{w}_{\mathbf{m}}$ can be expressed for the $N$-element array as,

$$
\mathbf{w}_{\mathbf{m}}=\left[\begin{array}{llll}
W_{m}\left(0, j \Omega_{t}\right) & W_{m}\left(1, j \Omega_{t}\right) & \ldots & W_{m}\left((N-1), j \Omega_{t}\right)
\end{array}\right]^{\top}
$$

Beamforming can be achieved by weighting and summing each of the antenna outputs as illustrated in Fig. 2.11. This weighting vector is given by,

$$
\alpha=\left[\begin{array}{llll}
\alpha_{0} & \alpha_{1} & \ldots & \alpha_{N-1}
\end{array}\right]^{\top}
$$

where $\left(\alpha_{0}, \alpha_{1}, \ldots, \alpha_{N-1}\right) \in \mathbb{C}$. This operation is equivalent to the implementation of a spatial filter where the z-domain transfer function is given by,

$$
H\left(z_{x}\right)=\sum_{k=0}^{N-1} \alpha_{k} z_{x}^{-k}
$$


By replacing the spatial $z_{x}$ variable with $e^{j \omega_{x}}$, where $\omega_{x}=\Delta x \Omega_{c t} \sin \psi$, the frequency response can be rewritten as,

$$
H\left(z_{x}\right)=\sum_{k=0}^{N-1} \alpha_{k} e^{-j \omega_{x} k}
$$

The output response $Y_{m}\left(j \Omega_{t}\right)$ for the input planar wave signal $w_{m}\left(n_{x}, t\right)$ can now be expressed as,

$$
Y_{m}\left(j \Omega_{t}\right)=\alpha \cdot \mathbf{Z} \cdot \mathbf{w}_{\mathbf{m}}
$$

The matrix given by $\mathbf{Z}$ is essentially an $N \times N$ diagonal matrix, where $z_{i, i}=e^{j \omega_{x} i}$. For narrowband cases, $\alpha_{i}$ coefficients in the weighting vector $\alpha$ become complex and the time domain output derived from Eqn. (2.31) simplifies to,

$$
y=\alpha^{\top} \mathbf{x}
$$

\subsubsection{FFT and TTD Beamforming}

The beam pattern generated in the far-field for a given phased antenna array is related to the discrete Fourier transform (DFT) of the spatial weighting factor vector $\alpha[85]$. Therefore, the weighting vector $\alpha$ defined in Eqn. (2.28) can also be expressed using $\alpha_{i}=e^{-j \Omega_{t} i \tau}$ as,

$$
\alpha=\left[\begin{array}{llll}
1 & e^{-j \Omega_{t} \tau} & \ldots & e^{-j \Omega_{t}(N-1) \tau}
\end{array}\right]^{\top}
$$

where, $\tau=\frac{\Delta x \sin \psi}{c}$. The term $e^{-j \Omega_{t} \tau}$ realizes a true time delay (TTD) across the signal bandwidth. Therefore, ideal realization of such a weight can produce a squintfree beam for wideband systems as shown in Fig. 2.11 (b). As the temporal frequency component does not spread too much from the carrier frequency $f_{c}$ for narrowband systems, $\Omega_{n b} \approx 2 \pi f_{c}$ and the term $e^{-j 2 \pi f_{c} \tau}$ becomes a complex constant. Equation (2.28) can be expressed for such a system as,

$$
\alpha=\left[\begin{array}{llll}
1 & e^{-j 2 \pi f_{c} \tau} & \ldots & e^{-j 2 \pi f_{c}(N-1) \tau}
\end{array}\right]^{\top} .
$$



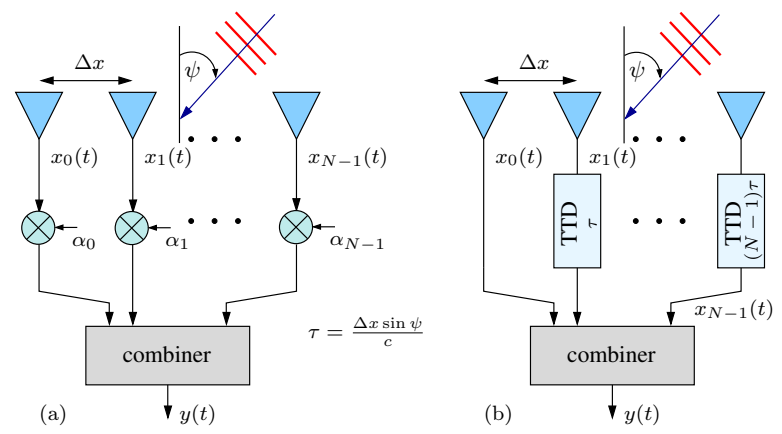

Figure 2.11: (a) Receive mode model for an $N$-element ULA with a wave impinging at DoA of $\psi$ and (b) the TTD and sum beamforming architecture.

The realization of multiple beams $M(<N)$ requires the extension of the weighting vector to a Vandermonde matrix of size $M \times N$ [86]. Each row of this matrix corresponds to a weighting vector realizing a beam at an angle of $\psi_{m}$, where $1 \leq m \leq M$. To achieve the highest number of degrees of freedom, each of the $M$ rows should be linearly independent and $M=N$ would give all the degrees of freedom. The $N$-point DFT matrix can be used to realize the $M=N$ beams, as the matrix is full ranked and captures all degrees of freedom. In other words, an $N$-point spatial DFT of an $N$-element narrowband phased antenna array would provide the maximum number of orthogonal beams.

Realization complexity of such DFT matrices in hardware can be simplified by fast Fourier transform (FFT) approaches. The upper-bound computational complexity, which is determined by the number of multiplications of the DFT matrix, is in the order of $\mathcal{O}\left(N^{2}\right)$; Cooley-Tukey FFT can reduce the complexity to the order of $\mathcal{O}\left(N \log _{2} N\right)$. However, the theoretical lower bound for the DFT multiplicative complexity was established as a function of $N$, where the lowest achievable complexity is $\mathcal{O}(N)$ [87], which can still be high for applications in mmWave, sub-THz systems and massive-MIMO. Chapter 7 includes a discussion of the possibility of approximating the DFT matrix for particular $N$ values in order to realize much greater efficiencies than at the theoretical lower bound. 


\section{CHAPTER 3}

\section{SPACE-TIME $\Sigma-\Delta$ MULTIPORT CIRCUITS}

In achieving our primary goal of reduction of ADC complexity in RF systems, we consider extending the well known $\Sigma-\Delta$ architecture to the MD spatio-temporal domain. This chapter discusses the possibility of implementing a single multiport ADC to replace the $N$-ADCs required in $N$-element phased array systems.

The idea of $\Sigma-\Delta$ modulation is based on the ideas of differential PCM and delta modulation, which were independently developed in research laborataries at International Telephone and Telegraph [88,89], Philips [90,91], and Bell Telephone Company [92]. In delta modulation, the input analog signal is quantized using a one-bit ADC (a comparator) (see Fig. 3.1 (a)). The comparator output is converted back to an analog signal using a 1-bit DAC, to be subtracted from the input after passing through the integrator. The systems transmits "1" to indicate that a positive excursion has occurred since the last sample and " 0 " to indicate that a negative excursion has occurred since the last sample. For a fixed DC input, the modulator generates an alternating pattern of $0 \mathrm{~s}$ and $1 \mathrm{~s}$.

Delta modulation does not have a theoretical limitation for the amplitude of the analog signal, as there is no limit to the number of pulses with the same sign to occur. However, unlike the case for a slowly varying signal (see Fig. 3.1 (b)),
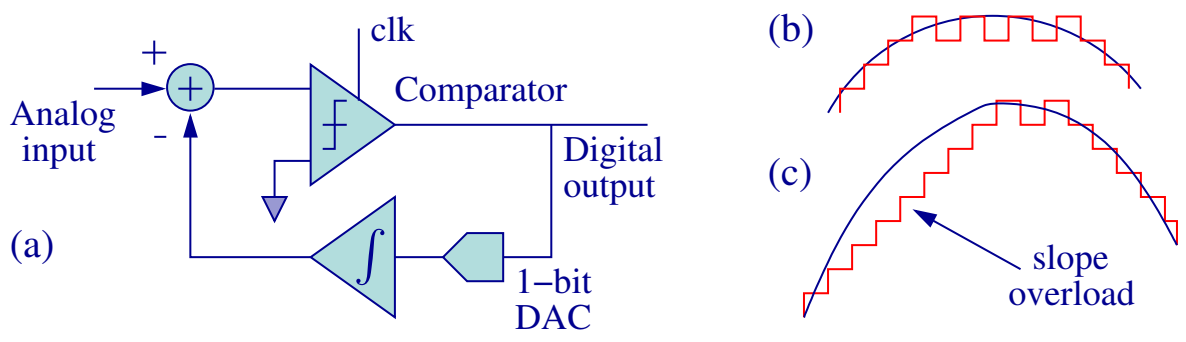

Figure 3.1: (a) Architecture of a delta modulator; quantization using a delta modulator for (b) slowly varying signals and (c) rapidly varying signals. 


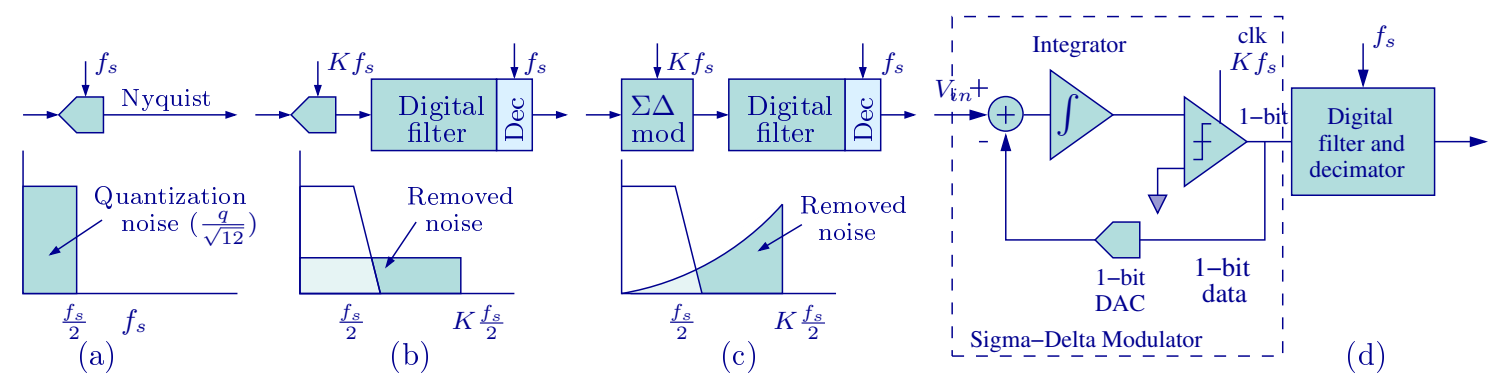

(a)

(b)

(c)

Figure 3.2: Comparison among the operation and quantization noise of (a) Nyquist sampling with traditional ADCs, (b) oversampling with traditional ADCs and (c) oversampling with $\Sigma-\Delta$ modulation. (d) First order $\Sigma-\Delta$ ADC.

the quantizer is unable to keep phase for a case where the signal varies rapidly as shown in Fig. 3.1 (c). The variation in the signal can be reduced by increasing the quantum step size and/or by increasing the sampling rate. However, delta modulation requires very high sampling rates (typically $20 \times$ highest frequency) as compared to the Nyquist rate of $2 \times$. However, a slight modification of the delta modulator leads to the $\Sigma-\Delta$ architecture, which is considered to be one of the most popular ADC architectures today.

\subsection{Background}

Quantization noise has a rms value of $q / \sqrt{12}$, which is uniform distributed within the Nyquist band (DC to $f_{s} / 2$ ) (see Fig. $3.2(\mathrm{a})$ ), where $q$ is the quantum step size and $f_{s}$ is the sampling frequency. As the quantization noise does not depend on the sampling rate, using a higher sampling rate (say $K f_{s}$ ) distributes the noise over a wider bandwidth of DC to $K f_{s} / 2$. Next, we can apply an LPF to the output to remove most of the quantization noise without affecting the desired signal as shown in Fig. 3.2 (b). A decimation block after the digital filter is used to convert the signal back to the original sampling rate. As $\mathrm{ENOB}=\frac{S Q N R_{d B}-1.76}{6.02}$, a reduction of quantization noise will increase ENOB [93,94]. In other words, a high-resolution 


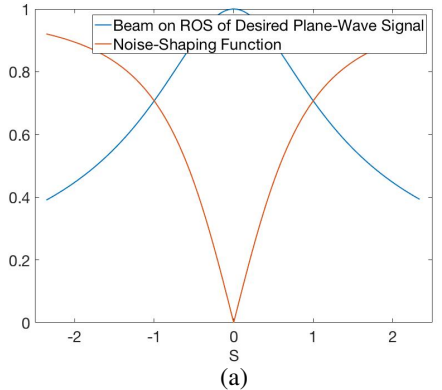

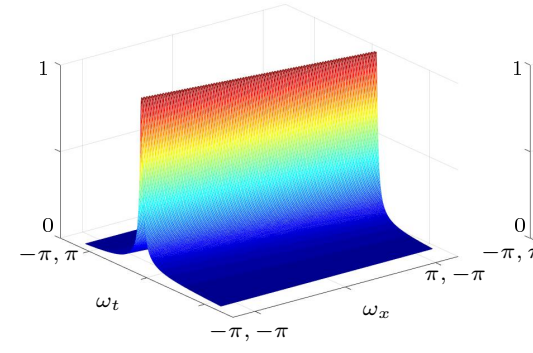

(b)

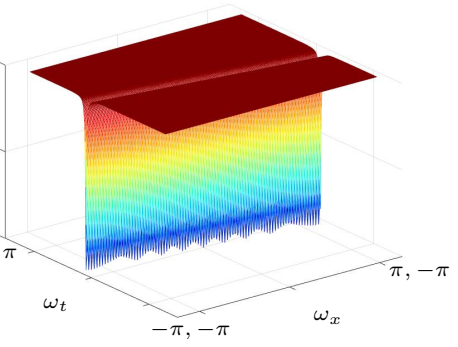

(c)

Figure 3.3: (a) 1D signal (low pass) and noise (high pass) transfer functions; (b) 2D space-time transfer function for the desired plane-wave signal; (c) 2D space-time transfer function for the unwanted quantization noise.

ADC can be achieved using a low-resolution ADC. However, in order to increase the ENOB by $N$ bits using this method, the oversampling factor has to be $2^{2 N}$. However, the use of a $\Sigma-\Delta$ modulator to replace the traditional ADC can reduce the requirement for a higher oversampling rate by shaping the quantization noise, such that most of it falls outside the passband, as shown in Fig. 3.2 (c).

The architecture of a first-order $\Sigma-\Delta$ ADC is shown in Fig. 3.2 (d). In the typical case, a feedback loop is used to achieve the following:

i. a low-pass response to the analog signal being sampled; and

ii. a high-pass response to the quantization noise injected by the quantizer into its digital outputs, which is known as noise-shaping (see Fig. 3.3 (a)).

A low-resolution quantizer is used to improve hardware efficiency, which results in a highly non-linear quantization process that injects a significant amount of harmonic distortion into the quantized samples. Fortunately, the $\Sigma-\Delta$ feedback loop also reduces the non-linearity of the system, which is known as distortion-shaping. As a result, $\Sigma-\Delta$ modulation allows low-precision quantizers to generate digital outputs with low levels of quantization noise and harmonic distortion. 


\section{2 $\Sigma-\Delta$ Concept in MD Signal Processing for ULAs}

Although used for ADCs, the mathematics of $\Sigma-\Delta$ conversions are not necessarily limited to ADC circuits. In fact, the $\Sigma-\Delta$ modulation technique can been applied in spatio-temporal MD circuits and systems found in antenna arrays, including LNAs. Work in [95-97] has shown that all types of electronic noise and non-linear distortion can be significantly reduced using spatio-temporal extensions of the $\Sigma-\Delta$ modulation principle.

The use of spatial over-sampling [95-98] leads to dense antenna arrays which, in turn, lead to MD spatio-temporal wave spectra that are artificially compressed into a smaller area in the 2D Nyquist region. In this section, we discuss the possibility of extending MD $\Sigma-\Delta$ algorithms for new applications in array processing to develop $N$-port ADCs to replace the traditional $N$ number of $M$-bit ADCs (see Fig. 3.5 (a)), as well as the possibility of eliminating the need for spatial over-sampling, such that there is no need to increase the number of antenna and transceiver circuits in the array processor. Elimination of spatial oversampling is efficient in terms of RF and digital hardware, since the number of array elements and transceiver circuits does not need to be increased in order to benefit from noise and distortion shaping.

In other words, extension of $\Sigma-\Delta$ architecture for MD also implies an extension of the high-pass filter applied on the signal and the low-pass filter applied on the quantization noise to 2D space-time domain as shown in Fig. 3.3 (b) and Fig. 3.3 (c) respectively.

To develop a model for $\Sigma-\Delta$ arrays, we begin by considering the (frequencyand bandwidth-normalized) temporal first-order $\Sigma-\Delta$ loop in the Laplace domain $s \in \mathbb{C}$. The corresponding block diagram and the mathematical model are shown in Fig. 3.4 (a) and Fig. 3.4 (b) respectively. 


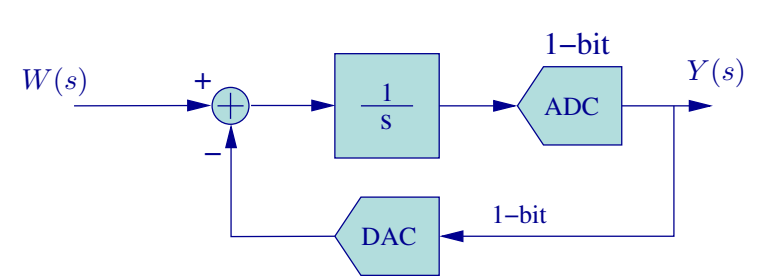

(a)

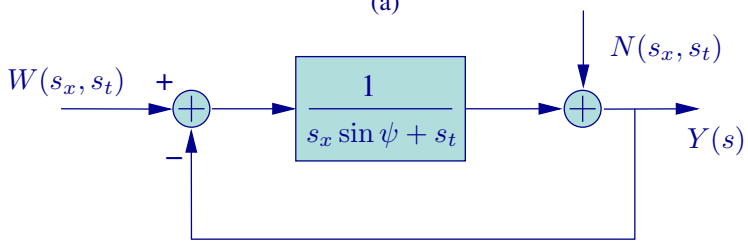

(c)

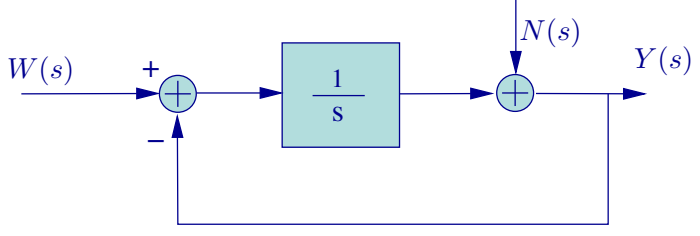

(b)

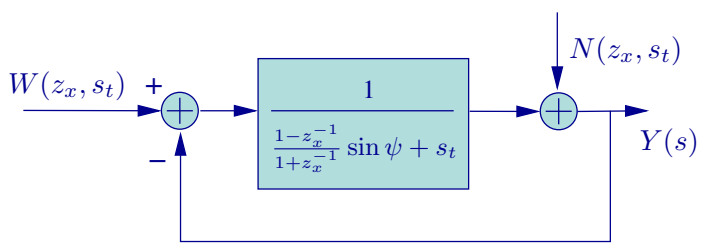

(d)

Figure 3.4: a) Feedback circuit for a conventional first-order $\Sigma-\Delta$ ADC; b) mathematical model for the ADC mentioned in a); c) 2D extension of the $\Sigma-\Delta$ loop where the linear-transform $s=s_{x} \sin \psi+s_{t}$ is applied; and d) mixed-domain 2D $\Sigma-\Delta$ loop where the bilinear transform $s_{x}=\frac{1-z_{x}^{-1}}{1+z_{x}^{-1}}$ is applied.

The temporally-oversampled analog input $W(s)$, digital output $Y(s)$, and quantization noise $N(s)$ (assumed to be additive and wideband) in the loop are related via

$$
Y(s)=W(s) \frac{1}{1+s}+N(s) \frac{s}{1+s} \in \mathbb{C} .
$$

This is the simplest possible $\Sigma-\Delta$ ADC; it has the following features:

i. a first-order low-pass transfer function $\frac{1}{1+s}$ for $W(s)$, and

ii. a first-order high-pass transfer function $\frac{s}{1+s}$ for $N(s)$.

Performance can be further improved by using more complex loops that apply higher-order low-pass and high-pass transfer-function pairs to the signal and quantization noise, respectively. Once sampled and quantized, the signal of interest is recovered using digital low-pass filtering followed by temporal down-sampling (decimation).

The received signal can be spatially oversampled by using closely spaced ULA. Since the spatial sample values are discrete, by applying the LDI transform given 


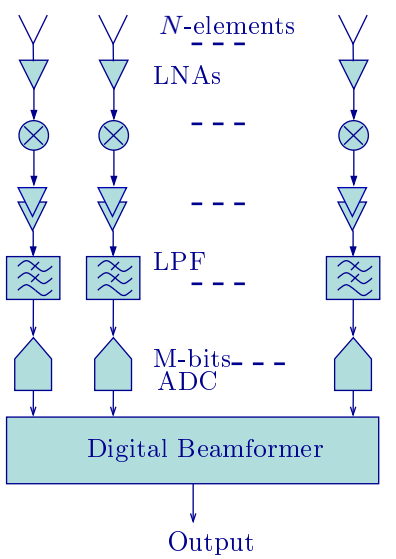

(a)

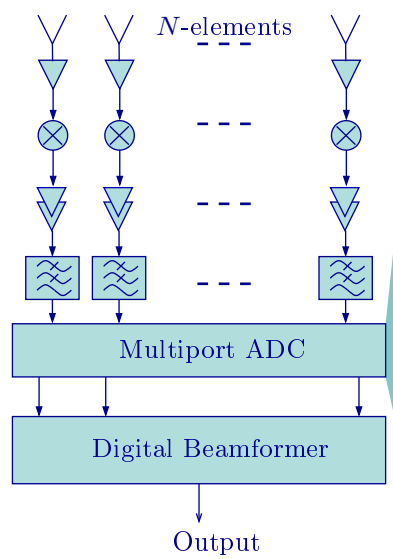

Output

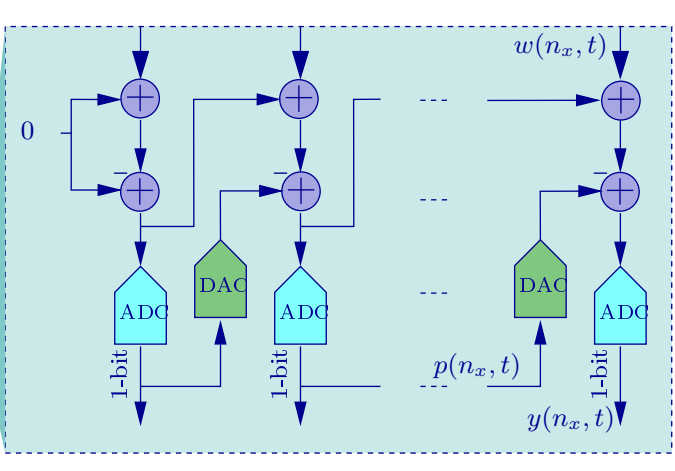

(b)

Figure 3.5: (a) Conventional receiver chain with $N$-bits ADCs, (b) proposed architecture with multiport ADC (1-bit).

by $\frac{1}{s}=\frac{z_{x}^{-1}}{1-z_{x}^{-1}}[99]$, we obtain the equations below:

$$
\begin{aligned}
& E\left(z_{x}\right)=W\left(z_{z}\right)-Y\left(z_{x}\right) \\
& Y\left(z_{x}\right)=P\left(z_{x}\right)+N\left(z_{x}\right) \\
& P\left(z_{x}\right)=\frac{z_{x}^{-1}}{1-z_{x}^{-1}} E\left(z_{x}\right)
\end{aligned}
$$

From Eqns. (3.2) and (3.3) the $Z$-domain output before quantization is obtained as,

$$
P\left(z_{x}\right)=z_{x}^{-1}\left[P\left(z_{x}\right)+W\left(z_{x}\right)-Y\left(z_{x}\right)\right]
$$

The difference equation for the multi-port ADC can now be written as,

$$
y\left(n_{x}, t\right)=Q\left[w\left(n_{x}-1, t\right)+p\left(n_{x}-1, t\right)-y\left(n_{x}-1, t\right)\right] .
$$

Implementation of the difference equation Eqn. (3.5) is shown in Fig. 3.5 (b).

The key point is that the mathematics of non-linear distortion/noise shaping are independent of the physics of its source. In our proposed array processing approach, the same $\Sigma-\Delta$ loop can be applied for any source of noise, whether it is ADC quantization noise, LNA thermal noise, mixer noise and intermodulation products, 
or power amplifier (PA) distortion products. By recognizing the fact that the $\Sigma-\Delta$ concept is agnostic to the source of noise, we are able to extrapolate it to the entire transceiver electronics for array apertures, covering LNA, mixer, PA, ADC, and DACs by using MD filtering and signal processing concepts. In our proposed approach, the conventional temporal-only $\Sigma-\Delta$ loop is expanded in dimensions of space and time and is realized using MD circuits and systems concepts.

This is a fundamentally new concept in antenna array aperture design, and it has the potential to offer performance improvements under certain conditions.

\section{Region of Support vs. Spatial Oversampling Factor}

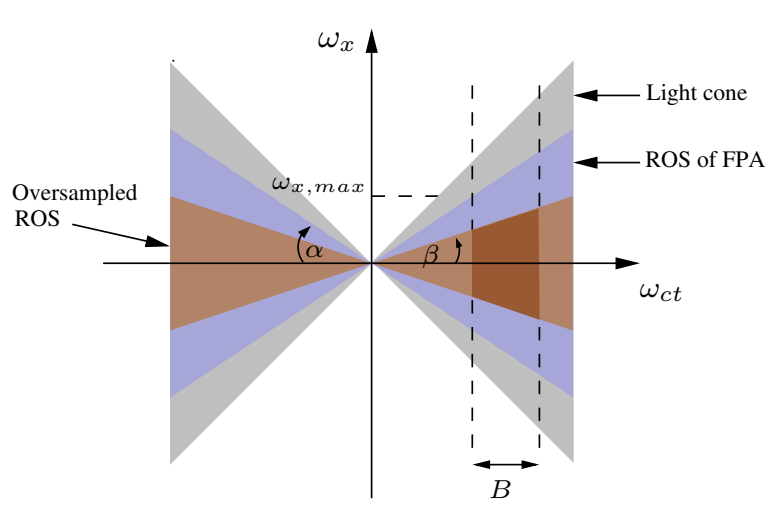

(a)

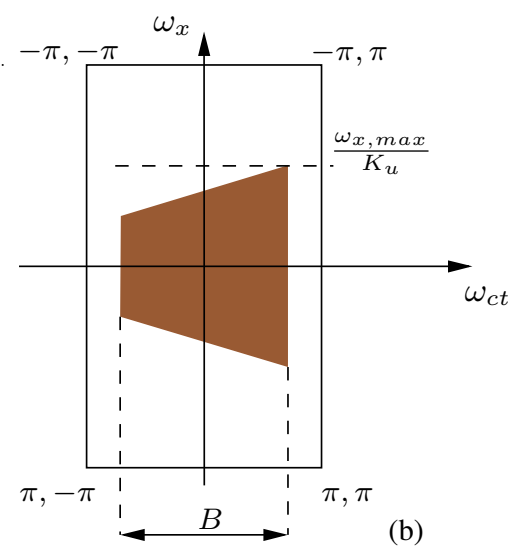

(b)

Figure 3.6: (a) Light cone, RoS and effect of oversampling. (b) Spectrum of the down-sampled signal.

The RoS of a signal received by a linear antenna array falls into the light cone which has a half-angle of $\pi / 4$. FPA reduces the RoS of the signal such that it will be entirely included in a cone with a half-angle of $\alpha$, where oversampling further reduces the angle to $\beta$, as shown in Fig. 3.6 (a). Down-sampling shifts the spectrum over the temporal frequency axis, and oversampling reduces the highest spatial frequency to $\frac{w_{x, \max }}{K_{u}}$ as shown in Fig. $3.6(\mathrm{~b})$. 


\subsubsection{Relaxation of Spatial Oversampling Requirement in}

\section{$\operatorname{MD} \Sigma-\Delta$ ADCs.}

The extension of the $\Sigma-\Delta$ concept to antenna arrays for improving the noise and distortion of the array transceiver electronics requires two important distinctions from the well-known two-port (input-output) ADC case:

i. The $\Sigma-\Delta$ algorithm now encompasses both spatial and temporal dimensions, and is therefore MD in nature; and

ii. While over-sampling is required for 2-port $\Sigma-\Delta$ ADCs, when the principle is extended to the MD spatio-temporal case, causality conditions for propagating

electromagnetic waves imply that the spatio-temporal spectra of the signals of interest have a well-defined RoS.

In fact, the spectral RoS of each wave is extremely sparse in the MD frequency domain, pertaining to direction and frequency variables (space and time). Such sparsity allows us to employ $\Sigma-\Delta$ in MD SFGs while removing the need for spatial and temporal over-sampling.

The objective of over-sampling is to make the signal of interest "low pass" compared to the "shaped noise", in order to separate them in the spectral domain. In the spatio-temporal domain pertaining to aperture arrays, we can "shape" the noise/distortion in spatio-temporal dimensions such that they are mutually exclusive (do not overlap) with the sparse RoS of the desired wave signals.

The first step in mapping a $\Sigma-\Delta$ modulator to a digital phased-array aperture is to include both time and space dimensions in the mathematical model. We can achieve this by adopting a linear transform of the type $s=s_{x} \sin \psi+s_{t}$ (see Fig. $3.4(\mathrm{c}))$ where $\left(s_{x}, s_{t}\right) \in \mathbb{C}^{2}$ are Laplace variables pertaining to the spatial 
frequency $\omega_{x}$ and the temporal frequency $\omega_{t}$, respectively, where the direction of propagation (launch angle) of the transmitted waves is given by $\psi \leq 90^{\circ}$ measured counter-clockwise from the broadside of the array. The proposed Laplace domain mapping leads to a $2 \mathrm{D}$ spatio-temporal realization of the $\Sigma-\Delta$ modulator for $0 \leq \psi \leq \frac{\pi}{2}$, which is given by,

$$
Y\left(s_{x}, s_{t}\right)=W\left(s_{x}, s_{t}\right) \frac{1}{1+s_{x} \sin \psi+s_{t}}+N\left(s_{x}, s_{t}\right) \frac{s_{x} \sin \psi+s_{t}}{1+s_{x} \sin \psi+s_{t}} .
$$

The realization of the proposed $2 \mathrm{D}$ spatio-temporal $\Sigma-\Delta$ modulator requires computing the algorithms only at locations where the antennas and transceivers are spatially located. We achieve the spatial discretization by employing the bilinear transform $s_{x}=\frac{1-z_{x}^{-1}}{1+z_{x}^{-1}} \in \mathbb{C}$. The resulting system (see Fig. $3.4(\mathrm{~d})$ ) is a 2D mixeddomain MD spatio-temporal $\Sigma-\Delta$ modulator having the transform equation

$$
Y\left(z_{x}, s_{t}\right)=W\left(z_{x}, s_{t}\right) \frac{1}{1+\frac{1-z_{x}^{-1}}{1+z_{x}^{-1}} \sin \psi+s_{t}}+N\left(z_{x}, s_{t}\right) \frac{\frac{1-z_{x}^{-1}}{1+z_{x}^{-1}} \sin \psi+s_{t}}{1+\frac{1-z_{x}^{-1}}{1+z_{x}^{-1}} \sin \psi+s_{t}} .
$$

This equation describes an aperture array that shapes away non-linearity/noise generated by the analog electronics. Because the proposed $\Sigma-\Delta$ algorithm is recursive in nature in both the spatial and temporal dimensions, the stability of the system is important. We utilize the theory of practical bounded-input boundedoutput (p-BIBO) stability of MD filters [100] to show that the system is stable for $0 \leq \psi<90^{\circ}$. To check p-BIBO stability [100], we first set $z_{x}=1$ and tested the $1 \mathrm{D}$ BIBO stability of the resulting system given by $Y\left(1, s_{t}\right)=W\left(1, s_{t}\right) \frac{1}{1+s_{t}}+$ $N\left(1, s_{t}\right) \frac{s_{t}}{1+s_{t}}$. The poles are on the left half of the s-plane, and therefore, BIBO stable. Then, we set $s_{t}=0$ and check the stability of the resulting $1 \mathrm{D}$ discrete system.

$$
Y\left(z_{x}, 0\right)=\frac{W\left(z_{x}, 0\right)}{1+\frac{1-z_{x}^{-1}}{1+z_{x}^{-1}} \sin \psi}+N\left(z_{x}, 0\right) \frac{\frac{1-z_{x}^{-1}}{1+z_{x}^{-1}} \sin \psi}{1+\frac{1-z_{x}^{-1}}{1+z_{x}^{-1}} \sin \psi} .
$$

The pole $z_{x}=\frac{\sin \psi-1}{1+\sin \psi}, 0 \leq \psi<90^{\circ}$ is real-valued and lies inside the unit circle $\left|z_{x}\right| \leq$ 1 (which ensures stability). Thus, the $2 \mathrm{D}$ system is guaranteed to be practically 
BIBO stable (p-BIBO stable). In addition, the proposed $\Sigma-\Delta$ algorithm can be thought of as a 2D p-BIBO stable extension of a classical resistively-terminated passive first-order inductance-resistance network [101].

(a)

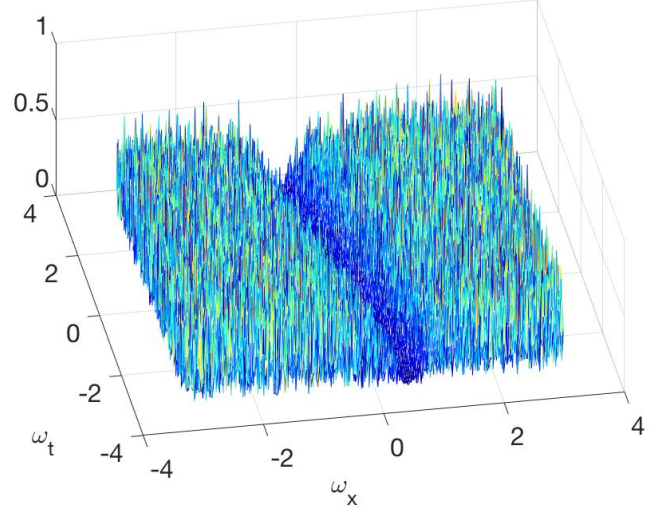

(b)

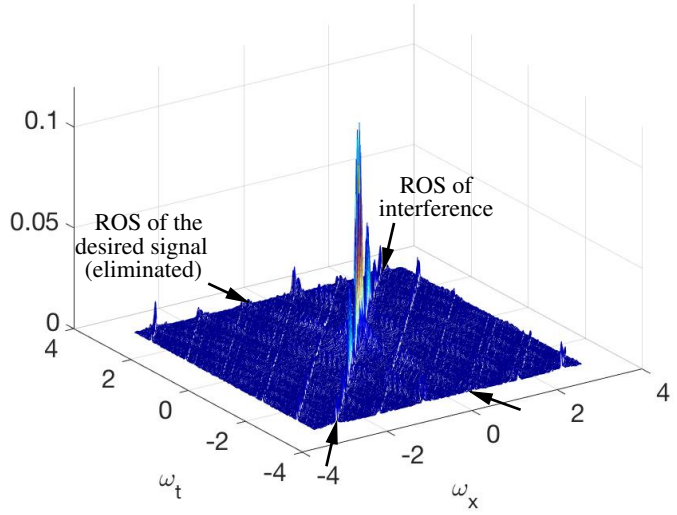

Figure 3.7: (a) The inclusion of the proposed 2D spatio-temporal $\Sigma-\Delta$ loop shapes the 2D noise such that the PSD of AWGN is significantly reduced along the lineshaped RoS of the desired RF signal; (b) in the proposed 2D $\Sigma-\Delta$ loop, the distortion components are shaped outside the beam shaped passband of interest.

The shaping of both additive white Gaussian noise (AWGN) and non-linear distortion by the proposed algorithm is explained using examples in Figs. 3.7. The AWGN in a conventional array occupies the entire frequency space in 2D (or in 3D, for rectangular arrays). However, plane waves that fall on the array are sparse in the 2D/3D frequency domain. Non-linear distortion generated by the electronics, especially due to quantization in the ADCs may lead to additional waves that overlap with the MD frequency-domain RoS of the input plane wave. Such overlap makes is impossible to use linear filtering to remove the undesired distortion components. Fortunately, noise shaping can greatly improve the signal-to-noise ratio of the array receiver.

In particular, the shaped noise power spectral density (PSD) of the proposed 2D $\Sigma-\Delta$ loop is close to zero along the chosen beam axis, as shown in Fig. 3.7 (a). That implies the received desired signal will be significantly less noisy than the 
undesired signal components. The digital beamformer that appears subsequent to 2D sampling removes the out-of-band noise without affecting the plane-wave input signal of interest. Similarly, distortion components are also greatly reduced along this axis (see Fig. 3.7 (b)). Therefore, the distortion due to quantization is significantly reduced for the passband of interest, while the quantization noise/distortion for undesired plane-waves still exist. Here, the distortion component for the planewave signal of interest (which falls on the primary RF beam of the antenna array) has effectly disappeared, leaving only the distortion of the out-of-band components. These components correspond to directions that are in the side-lobes of the subsequent digital beamformer and are, therefore attenuated in the final output signal. Thus, the proposed technique can effectively remove ADC non-linearities from the input plane wave of interest as long as it is aligned with the chosen beam axis. This concept is, to our knowledge, new to the signal processing literature.

\subsubsection{Implementation of the Proposed MD $\Sigma-\Delta$ Architec- ture}

A Conventional antenna array RF chain consists of $N$-bit ADCs, as shown in Fig. 3.8 (a). The use of high-resolution ADCs in receiver chain increases the hardware complexity. The proposed architecture eliminates the need to use multiple $N$-bits ADCs by introducing a multiport ADC with 1-bit quantization.

Implementation of the proposed MD $\Sigma-\Delta$ algorithm is best understood by considering the first-order 2D case. An antenna array processor realization of such a system based on receivers with dedicated direct-conversion electronics is shown in Fig. 3.8 (b); the algorithm has been applied to the ADCs. The use of Nyquist spacing in the spatial sampling operation requires the inter-element distance between 
antennas to be equal to $\Delta x=\lambda_{\min } / 2$, where $\lambda_{\min }$ is the free-space wavelength of the highest frequency wave that is expected to be received by the array. The necessary 2D analog beam filter consists of an active RF implementation denoted as $H\left(z_{x}, s_{t}\right)$. We will next explain how a suitable infinite impulse response (IIR) beam filter can be designed in a manner that can be implemented in high-speed integrated circuit (IC) technologies.

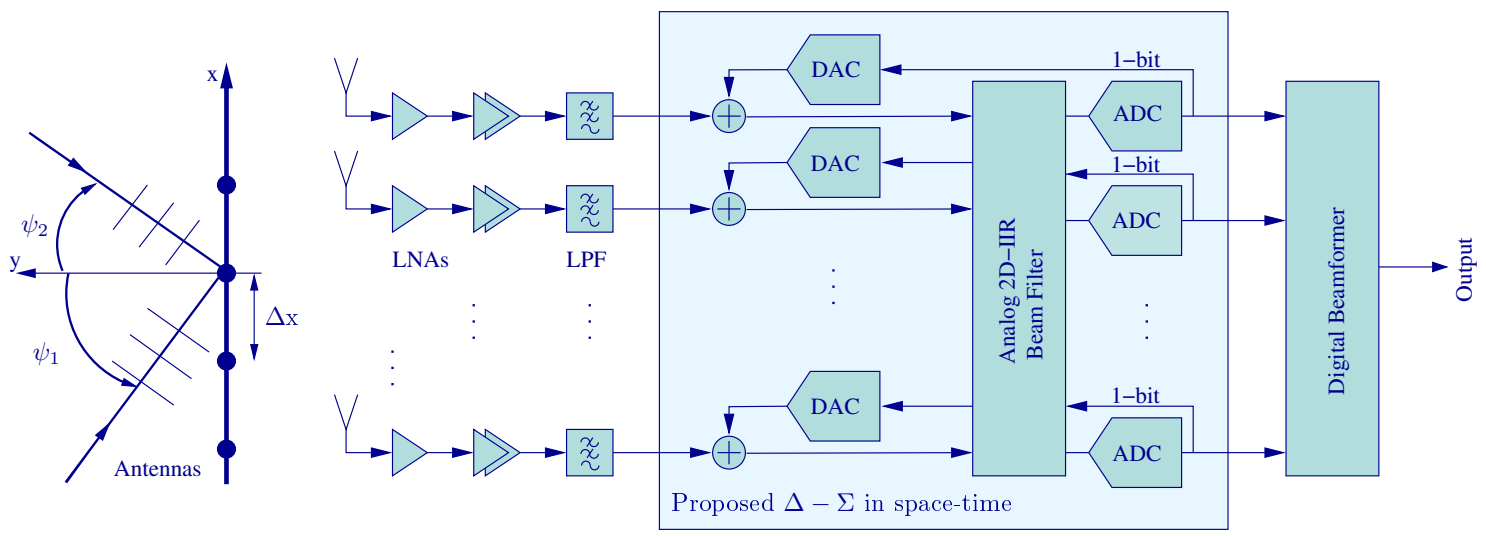

Figure 3.8: Conventional $N$-bit ADCs in the array receiver are replaced by spatiotemporal MD $\Sigma-\Delta$ ADCs. These form a multi-port analog-digital mixed-signal control system in which the forward path consists of a 2D-IIR analog beam filter that provides the 2D version of an ideal integrator $1 / s$. Quantization noise is thus removed from the passband beam that falls on the resonance region $s=0$. A highresolution beam can then be recovered by digital beamforming; quantization noise is shaped into the side-lobes of the beamformer and is thus attenuated.

The transfer function of the $2 \mathrm{D}$ beam filter used in the forward path of the 2D $\Sigma-\Delta$ modulator takes a direct-form mixed $z_{x}-s_{t}$ domain representation given by Eqn. (3.7). However, this representation can be complex and difficult to realize in analog RF-IC form. To address this problem, we propose the use of mixed differential-direct-form mixed-domain analog circuits, where the spatial interconnections between the different array processors are based on a differentialform operator and the temporal operations are maintained as direct-form operations. The spatial differential-form operator takes the mathematical form $z_{D}=$ 
$\frac{z_{x}^{-1}}{1+z_{x}^{-1}} \in \mathbb{C}[102]$. The forward beam transfer function can be written in the form $H\left(z_{x}, s_{t}\right) \equiv \frac{Y\left(z_{x}, s_{t}\right)}{W\left(z_{x}, s_{t}\right)}=\frac{1}{1+\alpha z_{D}+s_{t} / B}$, where $W\left(z_{x}, s_{t}\right)$ denotes the input signals from the antenna array, $\alpha=f(\sin \psi, R, T) \in \mathbb{R}$ with $f(., .,$.$) being a closed form rational$ function, and $B \in \mathbb{R}$ is the bandwidth of a first-order low-pass filter. To find the corresponding SFG, we change the $z_{x}$ transform variable back to the discrete spatial domain by computing an inverse spatial $z$-transform under zero initial conditions while maintaining the Laplace nature of the $2 \mathrm{D}$ filter (corresponding to the time dimension). Using well-known MD systems theory [101], we compute the inverse $z$-transform to obtain an array processor that uses a parallel realization of locally interconnected 2-input-2-output analog (i.e., non-quantized and non-sampled) modules to yield the final transfer function as shown in Fig. 3.9 (b). Thus, the 2D filtering operation is achieved by a locally interconnected array of $2 \mathrm{D}$ analog modules $(2 \mathrm{D}$ AMs) described using $y_{D}\left(n_{x}, t\right)=y\left(n_{x}-1, t\right)-y_{D}\left(n_{x}-1, t\right) \in \mathbb{R}$, where $y_{D}\left(n_{x}, s_{t}\right)$ is the continuous-time domain quantity corresponding to the output of the differential operation in space given in the $z_{x}$-domain as $z_{D}$. Taking the temporal Laplace transform of the above spatial differential operator, we obtain a $2 \mathrm{D}$ mixed-domain quantity $Y_{D}\left(n_{x}, s_{t}\right)=Y\left(n_{x}-1, s_{t}\right)-Y_{D}\left(n_{x}-1, s_{t}\right)$ that can be used to describe the primary signal flow path of the 2D AM using

$$
Y\left(n_{x}, s_{t}\right)=\frac{W\left(n_{x}, s_{t}\right)-\alpha Y_{D}\left(n_{x}, s_{t}\right)}{1+\frac{s_{t}}{B}} .
$$

A full description of the mathematical and circuit theoretical aspects of deriving the SFG can be found in [103]. A circuit realization of the SFG in the forward path provides the 2D spatio-temporal feedback necessary for MD noise and distortion shaping (see Fig. 3.8). In particular, the forward path consists of a linear combination of two quantities followed by a first-order low-pass filtering operation in the analog domain, as shown in Fig. 3.9 (a). It can be represented in the 2D mixed $z$-Laplace 
domain using a $2 \mathrm{D}$ extension of an ideal integrator, given by $\left(\frac{1-z_{x}^{-1}}{1+z_{x}^{-1}} \sin \psi+\frac{s_{t}}{B}\right)^{-1}$, where the bandwidth $B$ can reach several $\mathrm{GHz}$ for modern antenna arrays.

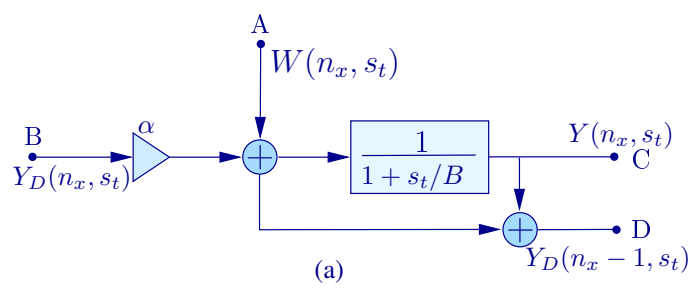

(b)

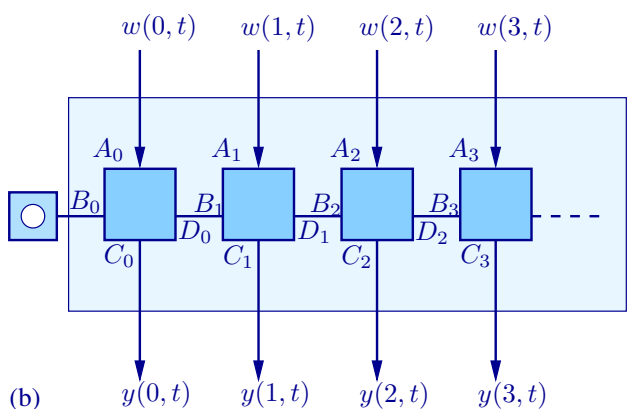

Figure 3.9: (a) SFG of a single 2D analog module (2DAM) used to realize the proposed IIR beam filter; (b) spatial interconnection of 2-input-2-output analog modules used to realize the proposed IIR beam filter.

\subsubsection{Applications of the Proposed Architecture}

Typical applications of the proposed MD $\Sigma-\Delta$ architecture include microwave and mm-wave imaging, wireless communications and emerging $5 \mathrm{G}$ wireless systems, phased-array radar, and radio astronomy instrumentation. The required operations can be realized over large bandwidths (multiple $\mathrm{GHz}$ ) using combinations of active and passive RLC elements in a complementary metal-oxide-semiconductor (CMOS) or bipolar-CMOS (BiCMOS) RF-IC technology. In the proposed system, the previously described $2 \mathrm{D}$ IIR analog beam filter is implemented in RF-IC form using CMOS circuits and embedded in a MD feedback loop such that the quantization noise generated by the 1-bit ADCs is shaped in both spatial and temporal frequency domains. The overall goal is for the RoS of the noise and distortion to lie outside the RoS of the signals of interest for far-field plane waves.

The receiver front-ends consist of LNAs having sufficient bandwidth and an NF low enough to meet application specifications. The amplified and low-pass filtered signals of each element in the antenna array must be digitized using a dedicated ADC 
for direct-conversion digital aperture array applications such as phased-array radars, radio-astronomy instrumentation, and mobile base stations for wireless communications. The digitization of the antenna array signals requires fast temporal sampling at rates greater than the Nyquist frequency, and the ADC resolution should be high enough to achieve sufficient linearity and low quantization noise. However, the relatively high bandwidth of RF applications necessitates ADC sampling rates in the range of hundreds of $\mathrm{MHz}$ to several $\mathrm{GHz}$. Such high sampling rate requirements necessitate the use of highly parallel ADC architectures such as flash converters. As mentioned earlier, a $N$-bit flash ADC requires $2^{N}$ parallel comparators, which indicates that chip area and power consumption grows exponentially with the resolution of each ADC. The proposed MD noise-shaping method allows the resolution of such converters to be greatly reduced (ideally, to 1-bit) without sacrificing ENOB and signal-to-noise-and-distortion ratio (SNDR), thus reducing both chip area and power consumption.

\subsection{Conclusion}

A 2D feedback topology is proposed based on a MD spatio-temporal extension of the $\Delta-\Sigma$ algorithm used in conventional high-resolution ADCs. By expanding the $\Delta-\Sigma$ concept to both space as well as time dimensions while discretizing space and keeping time continuous, it was shown that analog mixed-signal 2D IIR filters and low-resolution (ideally 1-bit) ADC/DAC pairs can be used in antenna array processing to effectively remove quantization noise from plane wave signals of interest. The proposed technique has been analyzed theoretically, and is believed to be suitable for experimental verification. Future high-bandwidth implementations of the concept will employ CMOS RF-IC technology. 


\section{CHAPTER 4}

\section{$\Sigma-\triangle$ CONCEPT IN MD SIGNAL PROCESSING FOR PARABOLIC DISH ANTENNA ARRAYS}

The extension of $\Sigma-\Delta$ to multidimensional systems, as discussed in Chapter 3, has possible applications in focal plane array (FPA) receiver systems. In this chapter, we will discuss the basics of FPA systems and the effects of spatial oversampling in $\Sigma-\Delta$ multidimensional extension for FPA systems.

Parabolic dish antennas and microwave lens antennas find a variety of applications in wireless communications, radar, electronic warfare, and radio astronomy $[104,105]$. Both dish antennas and microwave lens antennas possess a focal plane that is parallel to the aperture and going and passes through the prime focus of the dish/lens. The prime focus is typically used as the feed location containing a horn antenna or similar element such that the combined radiation pattern of the dish/lens and the feed horn provides a highly directional beam of RF energy [106]. A prime-focus-fed antenna provides a "pencil beam" pattern that is on-axis with the dish/lens. A dish/lens fed with a horn at the prime focus leads to a single pencil beam. However, the high cost of the antennas, as well as the requirement for more than one beam has led to the development of the FPA $[107,108]$.

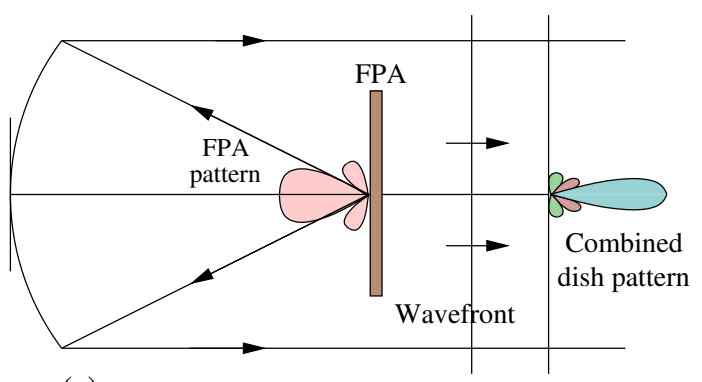

(a)

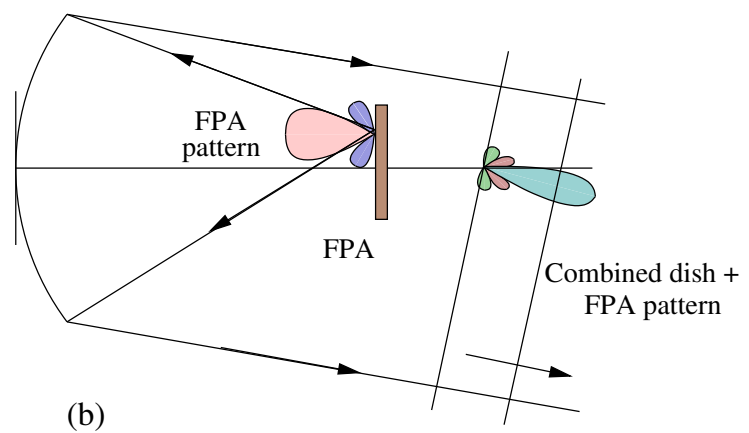

(b)

Figure 4.1: (a) Case 1: the prime-axis pencil-beam. (b) Case 2: an off-axis pencilbeam. 


\subsection{Background}

An FPA is an array of elements located on the focal plane of the dish/lens in a manner such that the electric and magnetic fields at several locations on the focal plane are sampled instead of only the prime focus $[109,110]$. Every point on the focal plane corresponds to an axis in a particular direction that contains its own pencil beam. Therefore, an $N$-element FPA provides $N$ individual RF pencil beams. It is possible to combine the signals from the $N$-elements using various phasing techniques, leading to the concept of a phased-array feed (PAF) where more than $N$ beams can be obtained, with better SNR than using a single element feed per beam. For additive white Gaussian noise (AWGN) conditions, the conjugate phase matching (CFM) method of finding the individual phases of the PAF network is best for finding the optimum SNR for a particular beam direction. The CFM PAF algorithm is effectively a matched filter based on finding the TTDs that coherently combine the signals from each PAF element for a particular focus spot on the focal plane. This chapter discusses the prime-axis pencil beam shown in Fig. 4.1 (a) in detail for simplified linear FPA. Extensions to off-axis pencil-beams shown in Fig. 4.1 (b) as well as rectangular FPAs will be considered in a future work.

Reflector antennas are known to achieve high gain and to have considerably lower manufacturing cost [107]. Use of phased array antennas facilitate electronic beam steering by manipulating the amplitude and phase distribution. A FPA has of two main components-a dish reflector and a 2D phased antenna array (as shown in Fig. 4.2 (a))-in which the above mentioned capabilities are combined. The antenna angle

$\alpha$ is defined as $\tan (\alpha)=\frac{d}{2 f^{\prime}}$ where $f^{\prime}$ is the distance between the focal plane and the dish and where $d$ is the diameter of the dish antenna (Fig. $4.2(\mathrm{~b})$ ). For a 3D sampled sequence of $w\left(n_{x}, n_{y}, n_{c t}\right)$ with a DOA of $(\psi, \phi)$ with respect to the array 


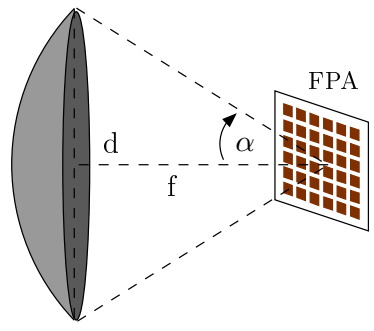

(a)

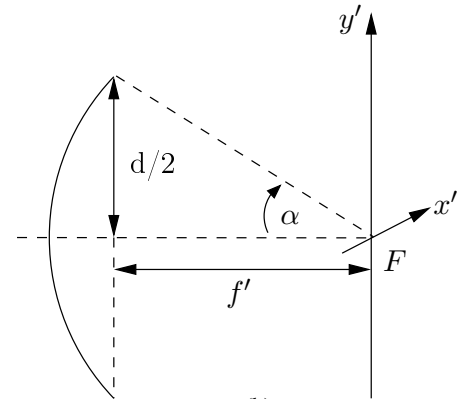

(b)

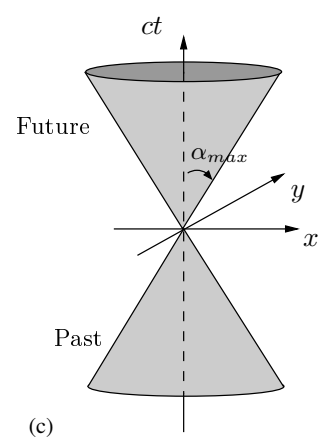

Figure 4.2: (a) Parabolic dish antenna, (b) notation used, (c) region of support of parabolic antenna.

surface, the spatio-temporal frequency spectrum, which is given by $W\left(\omega_{x}, \omega_{y}, \omega_{c t}\right)$, is entirely contained in a cone-shaped region with a half-angle of $\alpha$ as shown in Fig. 4.2 (c), given by Bruton and Bartley [101]:

$$
\left(\omega_{x}-\tan \theta_{i} \cos \phi_{i} \omega_{c t}\right)^{2}+\left(\omega_{y}-\tan \theta_{i} \sin \phi_{i} \omega_{c t}\right)^{2} \leq\left(\frac{\tan \epsilon \omega_{c t}}{\cos \theta_{i}}\right)^{2}
$$

Since the reflected signal on the FPA for a given planar wave is formed by signals arriving from $-\alpha$ to $+\alpha$, the corresponding $2 \mathrm{D}$ spectrum is no longer sparse. Therefore, spatial oversampling is required for this case, and implementation of the system follows Eqn. (3.5). Multiport ADC circuit realization is shown in Fig. 3.5 (b).

\subsubsection{Spatio-temporal $\Sigma-\Delta$ ADC}

In order to develop the spatio-temporal $\Sigma-\Delta$ architecture for noise shaping, we consider a frequency- and bandwidth-normalized, first-order $\Sigma-\Delta$ loop in the Laplace domain $s \in \mathbb{C}$, as shown in Fig. 3.4 (b). The analog input $W(s)$, quantized output $Y(s)$, and the additive wideband noise by the quanitzer $N(s)$ are related via $Y(s)=W(s) \frac{1}{1+s}+N(s) \frac{s}{1+s} \in \mathbb{C}$. It can clearly be seen that $\Sigma-\Delta$ architecture applies a low-pass transfer function on $W(s)$ and a high-pass transfer function on $N(s)$. 


\subsubsection{Simulation Results}

For the simulation in this study, an FPA with circular-aperture prime-focus paraboloidaldish reflector was assumed. The EM field formed by the incident wave can be calculated by considering the superposition of currents generated by an infinite number of points in the reflector. Hence, the ideal infinite extent focal EM field can be written as,

$$
e f r(x, y, z, t)=\sum_{\theta} \sum_{\phi} \hat{E}_{\theta, \phi} p \omega_{\theta, p h i}(x, y, z, t)
$$

where $\hat{E}_{\theta, \phi}$ is the polarization unit vector [76]. An FPA simulator whose design was based on this concept was used to simulate a wide band signal on the FPA. This system had a dish diameter $(d)$ of $5 \mathrm{~m}$, a focal length $(f)$ of $2.25 \mathrm{~m}$, and an array size of $32 \times 32$ elements. Cases of Nyquist spacing, $\times 2$ oversampling and $\times 4$ oversampling for a wideband signal between $2.1 \mathrm{GHz}-5.1 \mathrm{GHz}$ of bandwidth are considered.

\subsubsection{Noise Shaping}

A reflected signal from the dish antenna on a uniform linear array of 16 elements was selected to simulate noise shaping. Frequency domain spectrum of this signal is shown in Fig. 4.3 (a). Figures 4.3 (b) and 4.3 (c) show the effect of spatial oversampling, which squeezes the signal in the spatial frequency $\left(W_{x}\right)$ axis for oversampling factors of 2 and 4, respectively. A 1-bit quantizer with $\Sigma-\Delta$ architecture was used to quantize the considered wideband signal. Figures $4.3(\mathrm{~d}), 4.3$ (e) and 4.3 (f) show the quantization noise spectrum for the cases with no spatial oversampling, spatial oversampling with factor of $\times 2$, and spatial oversampling with factor of $\times 4$, respectively, where the quantization noise is shaped out of the ROS of the quantized signal. 


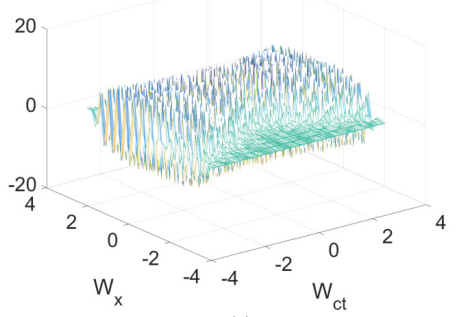

(a)

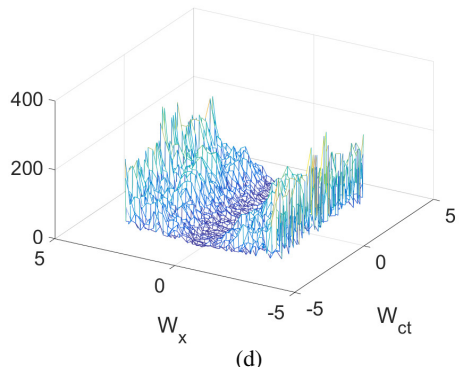

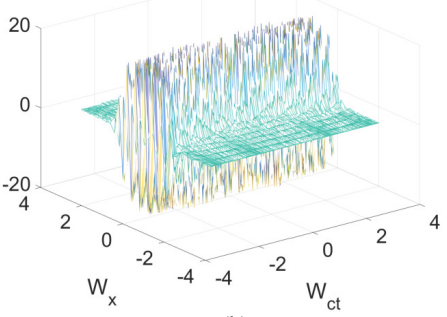

(b)

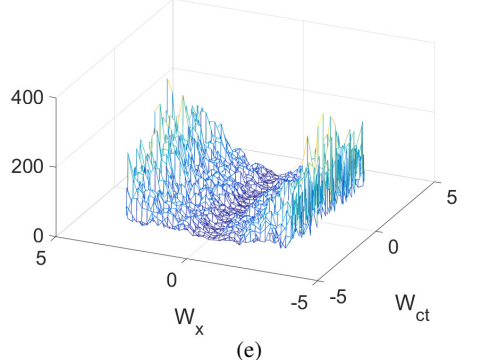

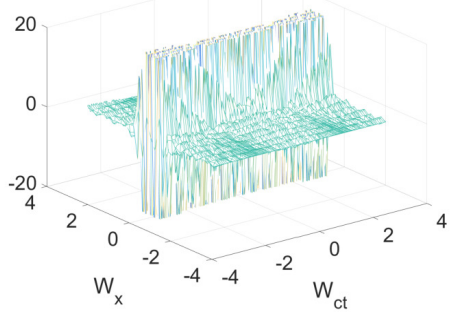

(c)

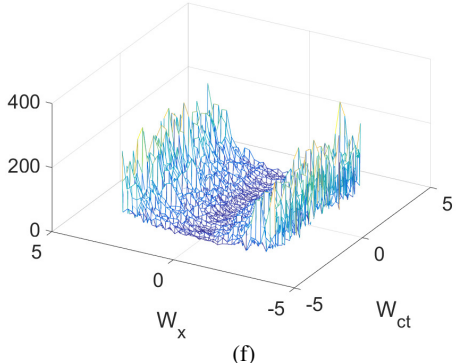

Figure 4.3: Spectrum in 2D: (a) wideband with no spatial oversampling, (b) wideband with spatial oversampling $\times 2$, (c) wideband with spatial oversampling $\times 4$; shaped noise for (d) wideband with no spatial oversampling, (e) wideband with spatial oversampling $\times 2$, (f) wideband with spatial oversampling $\times 4$.

\subsubsection{Improvements in Signal-to-Noise Ratio}

Figure 4.4 shows a signal-to-quantization-noise ratio (SQNR) comparison between quantization with proposed $\Sigma-\Delta$ architecture and normal quantization. Figures 4.4 (a), 4.4 (b), 4.4 (c) show the SQNR comparison results for cases from 1 to 3 bits for wideband signals with no spatial oversampling, oversampling $\times 2$, and oversampling $\times 4$, respectively. The results show that the SQNR improvement is significant for the case of 1-bit quantization and that spatial oversampling improves SQNR.

The spectrum with no spatial oversampling spans over the entire region of $(-\pi, \pi)$. Application of $\Sigma-\Delta$ quantization shapes the noise out of the ROS of the signal. A comparison of SNR values for normal quantization and $\Sigma-\Delta$ quantization is shown in Fig. 4.4 (a) for cases from 1 to 3 bits. 


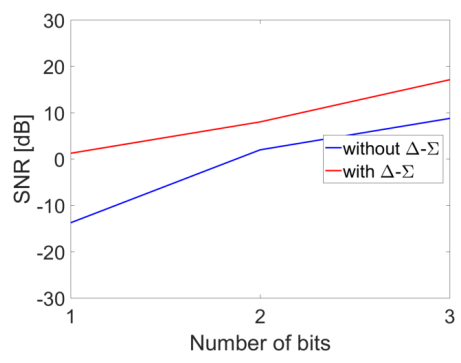

(a)

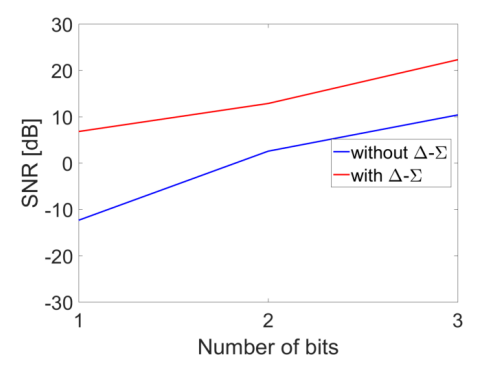

(b)

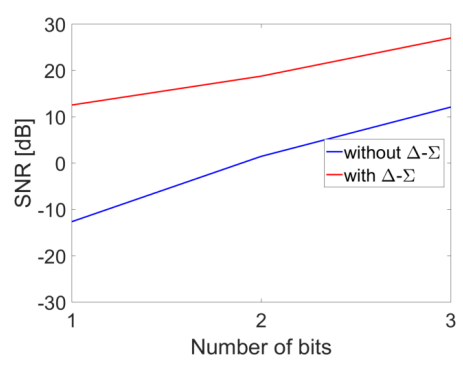

(c)

Figure 4.4: SNR improvements: (a) wideband with no spatial oversampling, (b) wideband with spatial oversampling $\times 2$, (c) wideband with spatial oversampling $\times 4$.

Oversampling of the wideband signal by a factor of 2 reduces the spatial frequency span of the signal such that the spectrum narrows down over the spatial frequency axis shown in Fig. 4.3 (b). This improves the SQNR as shown in Fig. 4.4 (b) for cases with 1 to 3 bits. Oversampling by a factor of 4 further squeezes the spectrum of the wideband signal over the spatial axis. Noise shaping on this signal improves the SQNR as shown in Fig. 4.4 (c) for cases with 1 to 3 bits.

\subsection{Conclusion}

A MD spatio-temporal extension of the $\Delta-\Sigma$ was proposed in this chapter for multiport ADCs for microwave focal plane array dish receivers. The extension involved modifying the conventional ADCs with the use of 1-bit quantizers. The proposed extension can be used for wideband signals with spatial oversampling to remove quantization noise. The proposed technique was analyzed using a simulation and it is believed to be suitable for experimental verification. 


\section{CHAPTER 5}

\section{ANALOG MULTIPORT NETWORK ALGORITHMS}

Chapter 2 discussed the spatio-temporal representation of a planar wave impinging on a uniform linear array (ULA) or a rectangular array and the correlation among the signal received by each element in the phased array. Einstein's causality light cone, discussed in Chapter 2, also showed that the 2D space-time frequency domain $\left(\omega_{x}, \omega_{c t}\right) \in \mathbb{R}^{2}$ is $50 \%$ empty even for the simplest case having a single spatial variable. That is, the RoS of all possible waves lies within the $2 \mathrm{D}$ projection of the light-cone of the frequency domain. For rectangular geometries (2D arrays), the frequency domain is a whopping $66 \%$ empty. Therefore, assigning a dedicated ADC for antenna/receiver ignores wave physics and treats the channels if they are uncorrelated. In this chapter, we will explore a suite of microwave circuits and digital signal processing (DSP) algorithms that exploit wave physics to drastically reduce the ADC count and overall complexity by packing information from multiple antenna elements into a single channel in a non-interfering manner.

We propose an array processing approach that exploits the the MD RoS of propagating plane-waves received from array antennas, which is constrained to a sparse region in the spatio-temporal frequency domain $\omega \equiv\left(\omega_{x}, \omega_{c t}\right) \in \mathbb{R}^{2}$. This chapter presents the theoretical and mathematical analysis of the proposed analog multiport network algorithms (AMNA) for ULAs, the process of extending these algorithms to rectangular apertures, and a discussion of the required hardware implementation.

\subsection{Theoretical Analysis of AMNA}

Practical antennas have constrained radiation patterns that act like spatial low-pass filters (Fig. 5.1(a)). The element patterns are constrained to the region $-\psi_{\max } \leq$ $\psi \leq \psi_{\max }$ for a predetermined gain threshold (e.g., $-10 \mathrm{~dB}$ ), such that the gain is 

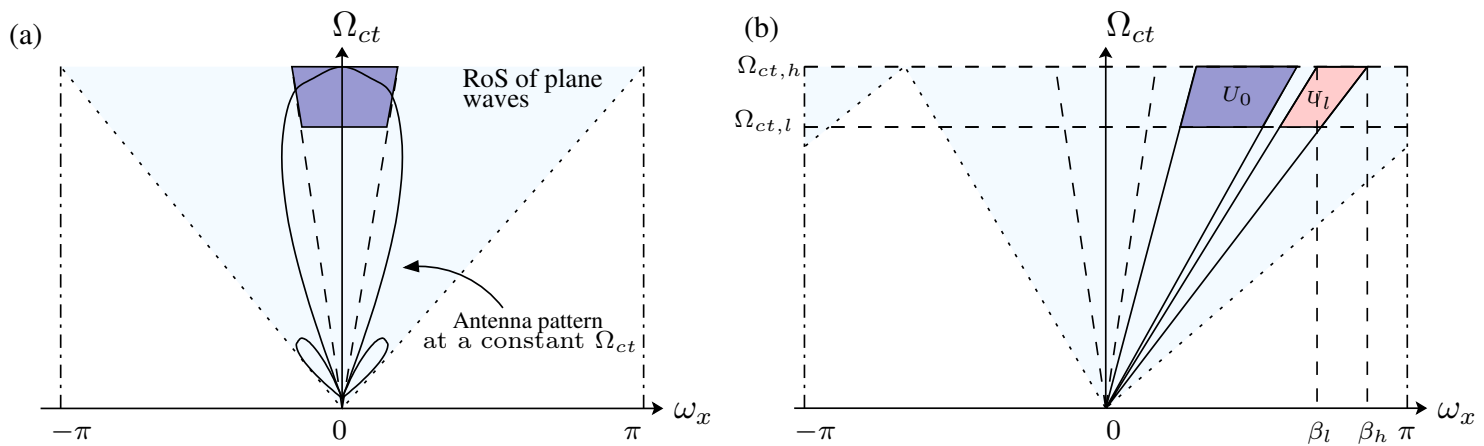

Figure 5.1: (a) Positive half (temporal) of the 2D frequency plane showing the finite element pattern; (b) Illustration of the linear transformed spectrum using the transformation matrix $\mathbf{V}$.

much lower than this threshold for $|\psi|>\psi_{\max }$. The resulting 2D spatio-temporal frequency domain is sparse, and the approximately empty space can be utilized to multiplex signals from multiple antenna elements (or polarizations) through a receiver chain. Such multiplexing occurs at a controlled level of interference that is part of the design trade-off for the system. The number of multiplexed signals can be increased to reduce the required number of front-ends and ADCs. Mathematically, this can be achieved by employing multidimensional linear transforms (MDLTs) to transform the spectra of different sub-arrays to minimally interfering regions of the MD domain of the spatio-temporal Fourier transform.

After the sparse regions of support are linear-transformed to lie on (ideally) mutually exclusive regions of the 2D spectral domain, they can be linearly combined without interference and sampled using one set of ADCs. Finally, the individual signals from the ULA can be recovered using a $2 \mathrm{D}$ digital filter. If the pattern per element (or sub-array) is constrained to be within $\pm \psi_{\max }=\pi / 2 K$, where $K \in \mathbb{Z}^{+}$, then the number of ADCs can be reduced by a factor of $K$ in the best case scenario. In this chapter this concept is referred to as "Analog Multiport Network Algorithms" or "AMNA". The theoretical analysis in this chapter was conducted in collaboration with Dr. Viduneth Ariyarathna [59]. 


\subsubsection{Mathematical Analysis}

If $W_{m}(\boldsymbol{\Omega})$ is the mixed domain 2D Fourier spectrum of the 2D mixed-domain plane wave denoted as $w_{m}(\boldsymbol{u}), \boldsymbol{\omega}=\left[\omega_{x}, \Omega_{c t}\right]^{\top}$, and if $\boldsymbol{u}=\left[n_{x}, c t\right]^{\top}\left(n_{x} \in \mathbb{Z}, c t \in \mathbb{R}\right)$, then the Fourier spectrum of $w_{m}(\mathbf{V} \boldsymbol{u})$ (in which $\mathbf{V}$ is an invertible $2 \times 2$ square matrix) is $\frac{W_{m}\left(\mathbf{V}^{-\top} \boldsymbol{\omega}\right)}{|\operatorname{det} V|}$, as proven below $[77,111]$.

Let $x_{a}(\mathbf{t})$ be a $2 \mathrm{D}$ analog signal where $\mathbf{t}=\left[t_{1}, t_{2}\right]^{\top} \in \mathbb{R}^{2}$ and let $\mathbf{V}$ be the sampling matrix made up of linearly independent sampling vectors $\mathbf{v}_{1}=\left[v_{11}, v_{21}\right]^{\top}, \mathbf{v}_{2}=$ $\left[v_{12}, v_{22}\right]^{\top}$, where $\mathbf{V}=\left[\mathbf{v}_{1} \mid \mathbf{v}_{2}\right]$. In this case, $\mathbf{t}=\mathbf{V n}$, where $\mathbf{n}=\left[n_{x}, c t\right]^{\top}, n_{x} \in \mathbb{Z}$, and $c t \in \mathbb{R}$. The spatially sampled, mixed-domain signal is then represented as $x_{m}(\mathbf{n})=x_{a}(\mathbf{V n})$. The original analog signal $x_{a}(\mathbf{t})$ satisfies the Fourier relationships

$$
\begin{aligned}
X_{a}(\boldsymbol{\omega}) & =\int_{-\infty}^{+\infty} x_{a}(\mathbf{t}) e^{-j \boldsymbol{\omega}^{\prime} t} d \mathbf{t}, \\
x_{a}(\mathbf{t}) & =\frac{1}{4 \pi^{2}} \int_{-\infty}^{+\infty} X_{a}(\boldsymbol{\omega}) e^{j \boldsymbol{\omega}^{\prime} t} d \boldsymbol{\omega} .
\end{aligned}
$$

Letting $\boldsymbol{\zeta}=\mathbf{V}^{\prime} \boldsymbol{\omega}$, the corresponding Fourier relationships for the sampled signal $x_{m}(\mathbf{n})$ are given by

$$
\begin{aligned}
& X_{m}(\boldsymbol{\zeta})=\sum_{n_{x}}\left[\int_{-\infty}^{+\infty} x_{m}(\mathbf{n}) e^{-j \Omega_{c t} c t} e^{-j \omega_{x} n_{x}} d \Omega_{c t}\right], \\
& x_{m}(\mathbf{n})=\frac{1}{4 \pi^{2}} \int_{-\infty}^{+\infty} \int_{-\pi}^{+\pi} X_{m}(\boldsymbol{\zeta}) e^{j \Omega_{c t} c t} e^{j \omega_{x} n_{x}} d \omega_{x} d \Omega_{c t} .
\end{aligned}
$$

Since $x_{m}(\mathbf{n})$ is obtained by sampling Eqn. (5.1), we can also write

$$
x_{m}(\mathbf{n})=x_{a}(\mathbf{V n})=\frac{1}{4 \pi^{2}} \int_{-\infty}^{+\infty} X_{a}(\boldsymbol{\omega}) e^{j \boldsymbol{\omega}^{\prime} \boldsymbol{V} n} d \boldsymbol{\omega} .
$$

Substituting $\boldsymbol{\zeta}=\mathbf{V}^{\prime} \boldsymbol{\omega}$,

$$
\begin{aligned}
x_{m}(\mathbf{n}) & =\frac{1}{4 \pi^{2}} \int_{-\infty}^{+\infty} \frac{1}{|\operatorname{det} \mathbf{V}|} X_{a}\left(\mathbf{V}^{-\top} \boldsymbol{\zeta}\right) e^{j \zeta^{\prime} n} d \boldsymbol{\zeta} \\
& =\frac{1}{4 \pi^{2}} \int_{-\infty}^{+\infty} \int_{-\pi}^{+\pi} \frac{1}{|\operatorname{det} \mathbf{V}|} \sum_{k} X_{a}\left(\mathbf{V}^{-\top}\left(\boldsymbol{\zeta}-2 \pi k \mathbf{e}_{1}\right)\right) e^{j \zeta^{\prime} n} e^{-j 2 \pi k \mathbf{e}_{1}^{\prime} \mathbf{n}} d \boldsymbol{\omega}
\end{aligned}
$$


where $\mathbf{e}_{1}=\left[\begin{array}{ll}1 & 0\end{array}\right]^{\top}, k \in \mathbb{Z}$, and the last term is unity. Thus,

$$
\left.x_{m}(\mathbf{n})=\frac{1}{4 \pi^{2}} \int_{-\infty}^{+\infty} \int_{-\pi}^{+\pi} \frac{1}{|\operatorname{det} \mathbf{V}|} \sum_{k} X_{a}\left(\mathbf{V}^{-\top}\left(\boldsymbol{\zeta}-2 \pi k \mathbf{e}_{1}\right)\right) e^{j\left(\Omega_{x} n_{x}+\Omega_{c t} c t\right.}\right) d \boldsymbol{\omega} .
$$

By comparing Eqn. (5.4) with Eqn. (5.2), we can see that

$$
X_{m}(\boldsymbol{\zeta})=\frac{1}{|\operatorname{det} \mathbf{V}|} \sum_{k} X_{a}\left(\mathbf{V}^{-\top}\left(\boldsymbol{\zeta}-2 \pi k \mathbf{e}_{1}\right)\right)
$$

Setting $\mathbf{V}=\left[\begin{array}{cc}1 & 0 \\ -c \tau & 1\end{array}\right]$, i.e., $\mathbf{V}^{-\top}=\left[\begin{array}{ll}1 & c \tau \\ 0 & 1\end{array}\right]$, would enforce a linear transform on the $2 \mathrm{D}$ signal by introducing a $n_{x} \tau, \tau \in \mathbb{R}$ delay to the signal at each spatial sample location $n_{x}$; here, $\tau$ would depend on $\frac{\Delta x \sin \psi_{\max }}{c}$. The linearly transformed spectra of $W_{m}\left(\mathbf{V}^{-\top} \boldsymbol{\omega}\right)$ (corresponding to the original $W_{m}(\boldsymbol{\omega})$ shown in green in Fig. 5.1(a)) are shown in Fig. 5.1(b). For an antenna array receiving a 2D plane wave, the received signal $s_{m}(\boldsymbol{u})$ and its spectrum $S_{m}(\boldsymbol{\omega})$ are filtered by the antenna response, where $S_{m}(\boldsymbol{\omega})=W_{m}(\boldsymbol{\omega}) \Psi(\boldsymbol{\omega})$ and where $\Psi(\boldsymbol{\omega})$ is the radiation pattern in the 2D Fourier domain.

The antenna pattern provides room for different spectra captured by equally spaced, equi-element sub-arrays to be shifted and combined together, thus reducing the number of required front-end chains. Alternatively, omni antennas may also be used under conditions of constrained field-of-view (FoV) with no strong directional interference outside the expected FoV. For example, radio astronomy arrays may look up at the sky in a $30^{\circ} \mathrm{FoV}$, or a long-range radar receiver may sense within a cone of interest. The signal energy of the spectrum $S_{m}(\boldsymbol{\omega})$ within the frequency band $U_{l}$ (in Fig. 5.1(b)) is given by

$$
I_{l, 0}=\frac{1}{4 \pi^{2}} \iint_{U_{l}} \frac{1}{|\operatorname{det} \mathbf{V}|^{2}}\left|S_{m}\left(\mathbf{V}^{-\top} \boldsymbol{\omega}\right)\right|^{2} d \boldsymbol{\omega}
$$

If $U_{l}$ is defined by $\omega_{x}=\left[\beta_{l}, \beta_{h}\right]$, the corresponding $2 \mathrm{D}$ spectral region is defined by the intersection of four lines: $\Omega_{c t}=\Omega_{c t, h}, \Omega_{c t}=\Omega_{c t, l}, \Omega_{c t}=\frac{\Omega_{c t, h}}{\beta_{l}} \omega_{x}$, and $\Omega_{c t}=\frac{\Omega_{c t, h}}{\beta_{h}} \omega_{x}$. 
As a result, Eqn. (5.6) can be written as

$$
I_{l, 0}=\frac{1}{4 \pi^{2}} \int_{\Omega_{c t, l}}^{\Omega_{c t, h}} \int_{\frac{\beta_{l} \Omega_{c t}}{\Omega_{c t, h}}}^{\frac{\beta_{h} \Omega_{c t}}{\Omega_{c t, h}}} \frac{1}{|\operatorname{det} \mathbf{V}|^{2}}\left|W_{m}\left(\mathbf{V}^{-\top} \boldsymbol{\omega}\right) \Psi\left(\mathbf{V}^{-\top} \boldsymbol{\omega}\right)\right|^{2} d \boldsymbol{\omega} .
$$

\subsubsection{Extension to $K$ Bands with Different Linear Trans-}

\section{forms}

Suppose we use different transformations $\mathbf{V}_{k}=\left[\begin{array}{cc}1 & 0 \\ -c \tau_{k} & 1\end{array}\right], k \in[0, K-1]$ to transform signals from antenna elements (or sub-arrays of identical spacing and size) in a ULA. The idea is to choose matrices $\mathbf{V}_{k}$ such that all $S_{m, k}\left(\boldsymbol{V}_{k} \boldsymbol{u}\right)$ s are combined to make

$$
y_{m, k}(\boldsymbol{u})=\sum_{k=0}^{K-1} S_{m, k}\left(\boldsymbol{V}_{k} \boldsymbol{u}\right)
$$

such that the required ADC complexity can be reduced by a factor of $K\left(K \in \mathbb{Z}^{+}\right)$ while also minimizing the interference between different spatial bands. The spectrum of the combined mixed-domain 2D signal after the linear transformation is

$$
Y_{m}(\boldsymbol{\omega})=\sum_{k=0}^{K-1} S_{m, k}\left(\mathbf{V}_{k}^{-\top} \boldsymbol{\omega}\right)
$$

where $S_{m, k}\left(\mathbf{V}_{k}^{-\top} \boldsymbol{\omega}\right)$ is the spectrum of the linearly-transformed 2D signal from the $k$ th sub-array. Suppose $\mathbf{V}_{k=l}$ is used to transform the signal of the $l^{\text {th }}$ sub-array

such that the dominant spectrum of the signal $S_{m, k}\left(\mathbf{V}_{l}^{-\top} \boldsymbol{\omega}\right)$ is moved to the spatial frequency band $U_{l}$. The interference energy that is artificially created in the $U_{l}$ band can now be calculated as follows:

$$
\begin{gathered}
I_{l}=\frac{1}{4 \pi^{2}} \int_{\Omega_{c t, l}}^{\Omega_{c t, h}} \int_{\frac{\beta_{l} \Omega_{c t}}{\Omega_{c t, h}}}^{\frac{\beta_{h} \Omega_{c t}}{\Omega_{c t, h}}} \mid \sum_{\substack{k=0 \\
k \neq l}}^{K-1}\left[\frac{1}{\left|\operatorname{det} \mathbf{V}_{\mathbf{k}}\right|} W_{m, k}\right. \\
\left.\left(\mathbf{V}_{\mathbf{k}}{ }^{-\top} \boldsymbol{\omega}\right) \Psi_{k}\left(\mathbf{V}_{\mathbf{k}}{ }^{-\top} \boldsymbol{\omega}\right)\right]\left.\right|^{2} d \boldsymbol{\omega} .
\end{gathered}
$$



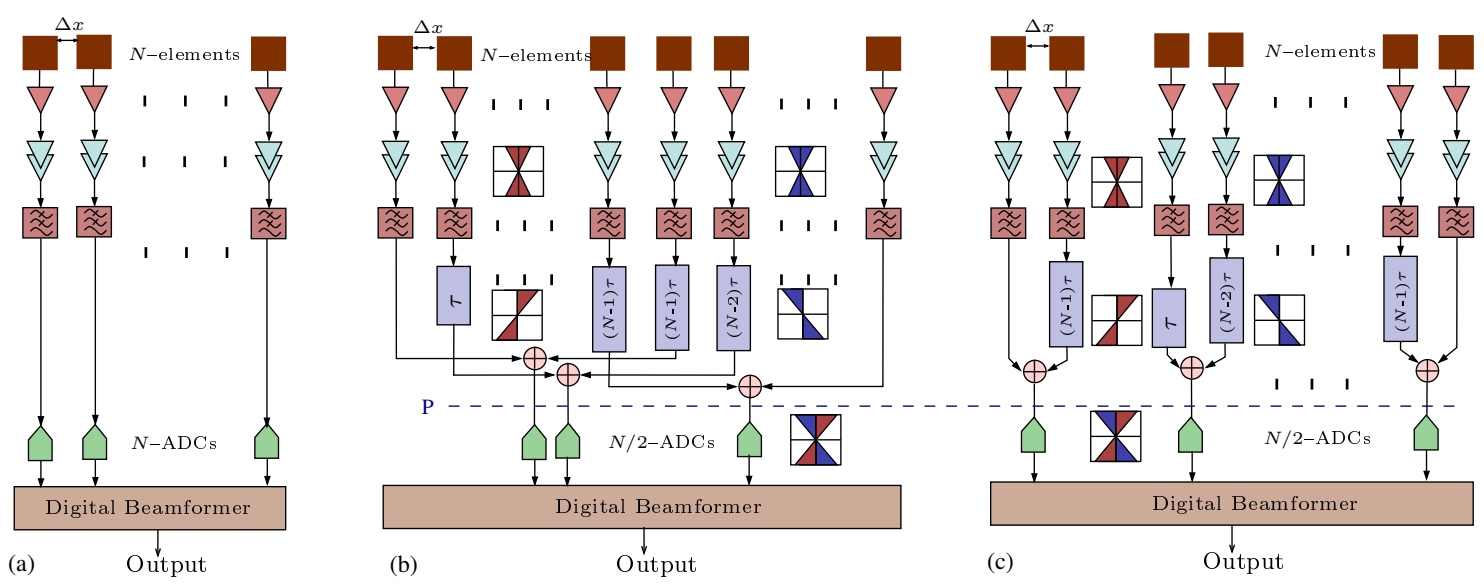

Figure 5.2: (a) Conventional architecture of an all-digital beamforming array. (b) Overview architecture of the MDLT method, in which linear-transformed spectra are combined to reduce the ADC count. (c) The intact MD spectrum observed by the ULA; linear-transformed spectra for (d) Sub-array 1, and (e) Sub-array 2.

The model in Eqn. (5.10) can now be used to derive the distortion introduced by the added signal processing by substituting the analytical or simulated antenna radiation patterns.

\subsubsection{Simulated Example: ULA}

Consider an $N$-element Nyquist-sampled ULA (shown in Fig. 5.2(a)) where the antenna patterns are restricted to $0<\psi<\frac{\pi}{6}$, such that the RoS in the MD spectrum is constrained to be within $-\tan ^{-1} \frac{1}{2} \leq \theta \leq \tan ^{-1} \frac{1}{2}$ as shown in Fig. 5.2(c). To apply the proposed MDLT, the ULA is divided into two sub-arrays, as shown in Fig. 5.2(b), without changing the inter-element spacing. The linear transform shifts the MD spectra of Sub-array 1 and Sub-array 2 to the left and right respectively, as shown in Figs. 5.2(d-e), such that the signals can be combined without spectral interference as shown in Fig. 5.2(f). 


\subsection{Extension to Rectangular Apertures}

Consider an $N_{x} \times N_{x}$ Nyquist-sampled rectangular aperture in the far-field shown in Fig. 2.7. By extending the mathematical analysis to the mixed-domain 3D Fourier spectrum $W_{m}(\boldsymbol{\omega})$, where the $3 \mathrm{D}$ mixed-domain plane wave is denoted as $w_{m}(\boldsymbol{u})$, $\boldsymbol{\omega}=\left[e^{-j \omega_{x}}, e^{-j \omega_{y}}, \Omega_{c t}\right]^{\top}$ and $\boldsymbol{u}=\left[n_{x}, n_{y}, c t\right]^{\top}\left(n_{x}, n_{y} \in \mathbb{Z}\right.$ and $\left.c t \in \mathbb{R}\right)$, the Fourier spectrum of $w_{m}(\mathbf{V} \boldsymbol{u})$, (where $\mathbf{V}$ is an invertible $3 \times 3$ square matrix) is $\frac{W_{m}\left(\mathbf{V}^{-\top} \boldsymbol{\omega}\right)}{|\operatorname{det} V|}$. For $\mathbf{V}=\left[\begin{array}{ccc}1 & 0 & 0 \\ 0 & 1 & 0 \\ -\tau_{x} & -\tau_{y} & 1\end{array}\right]$, i.e., $\mathbf{V}^{-\top}=\left[\begin{array}{ccc}1 & 0 & \tau_{x} \\ 0 & 1 & \tau_{y} \\ 0 & 0 & 1\end{array}\right]$, the linear transform extends to 3D such that the temporal frequency remains unaffected, while the spatial frequencies are shifted to

$$
\omega_{x}^{\prime}=\omega_{x}+\tau_{x} \Omega_{c t}, \omega_{y}^{\prime}=\omega_{y}+\tau_{y} \Omega_{c t}, \text { and } \Omega_{c t}^{\prime}=\Omega_{c t},
$$

where $\tau_{x}$ depends on $\frac{\Delta x \sin \theta \cos \psi}{c}$ and $\tau_{y}$ depends on $\frac{\Delta y \sin \theta \sin \psi}{c}$. The transformed mixed-frequency domain takes the form $\left[e^{-j \omega_{x}^{\prime}}, e^{-j \omega_{y}^{\prime}}, \Omega_{c t}\right] \in \mathbb{C}^{2} \mathbb{R}$.

Assume the antenna patterns are restricted to $0<\theta<\theta_{\max }$ for all $\phi$. The RoS of the spectrum observed by the rectangular aperture is constrained by a cone $\omega_{x}^{2}+\omega_{y}^{2}=\left(K \Omega_{c t}\right)^{2}$, as illustrated in Fig. 5.3(a). The linear transform slants the conical region of the RoS to a different direction while following the equation $\left(\omega_{x}^{\prime}-\right.$ $\left.\tau_{x} \Omega_{c t}^{\prime}\right)^{2}+\left(\omega_{y}^{\prime}-\tau_{y} \Omega_{c t}^{\prime}\right)^{2}=\left(K \Omega_{c t}^{\prime}\right)^{2}$. Such a linearly transformed spectrum for a rectangular aperture is shown in Fig. 5.3(b). Linear transforms can be used in different configurations, as shown in Figs. 5.3(c-d), to reduce the number of ADCs required to sample the receiver chains. 


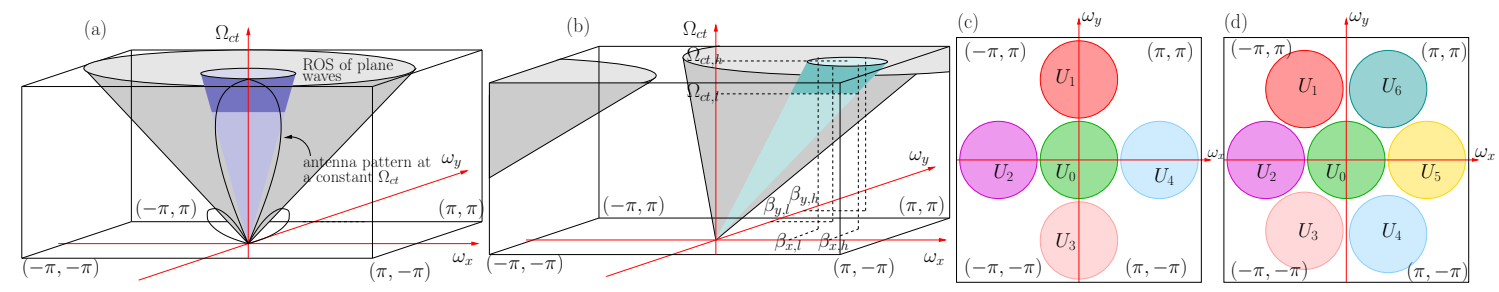

Figure 5.3: (a) The spectrum observed by the rectangular aperture, and (b) linear transformed spectrum. Possible linear transform configurations to combine (c) five, and (d) seven channels.

\subsubsection{Simulated Example: URA}

The uniform rectangular array (URA) in Fig. 5.4(a) is considered for simulation. The simulated RoS of the input spectrum seen by the URA takes the form of a frustum bounded by the light cone, as shown in Fig. 5.4(c). A $2 \mathrm{D}$ matrix of $\tau$ is considered for the convenience of illustration, such that $\tau_{x, y}=\tau_{x}+\tau_{y}$ where $(x, y)$ are the coordinates corresponding to each antenna location $\left(n_{x}, n_{y}\right)$. This configuration considers four sub-arrays (one for each quadrant) that are defined as shown in Fig. 5.4(a). Four different linear transforms are applied to the RF signals from these sub-arrays. These linear-transformed signals are combined without interference, processed by RF chains for amplification and down-conversion, and sampled using ADCs as shown in Fig. 5.4(b).

Our simulations assumed $0<\theta<\pi / 6$ for a Nyquist-spaced URA with $M \times N$ elements. The spectrum of sub-array a (red) was shifted to the second quadrant by applying delays over both rows ( $\tau_{y}$ steps) and columns ( $\tau_{x}$ steps $)$ starting from the top left corner, whereas the spectrum of sub-array $b$ (blue) was shifted to the first quadrant (on $\omega_{x}-\omega_{y}$ plane) by applying increasing delays over rows $\left(\tau_{y}\right.$ steps) and decreasing delays over columns ( $\tau_{x}$ steps). The spectra of sub-arrays $c$ and $d$ were transformed to the third and fourth quadrants, respectively. These signals can be combined using a $K: 1$ combiner without spectral overlap (Fig. 5.4(d)), thus 

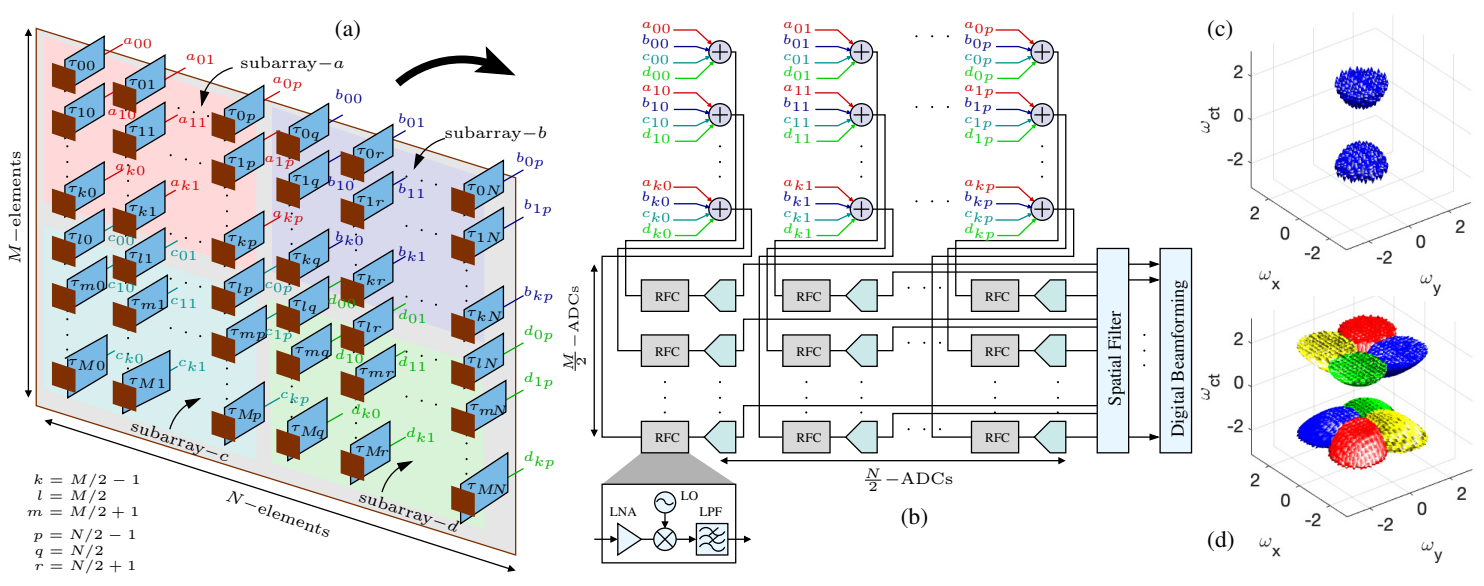

Figure 5.4: (a) Uniform rectangular array (URA) with MD linear transforms at $\mathrm{RF}$, and (b) combination stage followed by RF chains (RFCs) and ADCs for a fully digital beamformer. Simulated spectra: (c) observed by the rectangular aperture, and $(\mathrm{d})$ linear-transformed and combined without interference.

allowing the ADC count to be reduced by a factor of $K=4$. As a result, ADC complexity is reduced by $75 \%$.

\subsection{Hardware Implementation}

The linear transforms are realized using passive transmission line segments, with lengths chosen to provide the required true time delays within the frequency bands of interest. The amplified output of each LNA is applied to a set of transmission lines. The delayed signal components are combined using passive microwave power combiners (based on microstrip lines or transformers) before being applied to the ADCs.

Due to their use of low-loss delay elements, the linear transforms will not significantly affect receiver noise figure. However, the increased signal power present at each ADC input will reduce the effective number of bits (ENOB) of the final sam-

pled and quantized received signal on a per-antenna basis. Specifically, the peak signal amplitude from each $K$-fold multiplexed component must be reduced by a 
factor of $K$ to prevent ADC saturation. Thus, multiplexing will reduce each ADC's peak signal-to-noise and distortion ratio (SNDR) by $20 \log _{10}(K) \mathrm{dB}$, and ENOB will degrade by 1 bit each time the value of $K$ is doubled. Such degradation may not be significant, since low-resolution ADCs (down to 1-bit resolution) are becoming more popular for wireless systems [112].

\subsection{Conclusion}

The conventional use of ADCs in receiver systems assumes omni-directional antenna patterns, which are directional in practice and waste resources due to the sampling of unoccupied regions in the 2D spectral domain. This chapter explained how we exploited directional sparsity of directional antenna arrays by using a linear transformation, such that the unoccupied region of the $2 \mathrm{D}$ spectrum can be used to include information from multiple antennas/sub-arrays. The mathematical analysis shows that the ADC requirement can be reduced by at least $50 \%$ for ULAs and by $75 \%$ for URAs without losing array degrees of freedom. 


\section{CHAPTER 6}

\section{RECEIVER APPROACHES BASED ON DIFFERENT MULTIPLEXING METHODS}

In previous chapters, we used multidimensional spectral properties to reduce the $\mathrm{ADC}$ requirement in RF systems. In this chapter, we will discuss the possibility of time-division, code-division and frequency-division multiplexing (TDM, CDM AND

FDM, respectively) in order to combine outputs from multiple receiver antennas into a single ADC.

The advent of RF-enabled digital hardware, such as the Xilinx RF system on chip (SoC) technology, allows the combined realization of programmable digital fabrics with high-speed ADC/DAC on the same chip [113]. An RF SoC platform such as Xilinx ZCU 1285 platfrom (shown in Fig. 6.4 (a)) contains 16 high-speed ADCs $(\approx 2 \mathrm{GS} / \mathrm{s})$ and 16 high-speed DACs $(6.5 \mathrm{GS} / \mathrm{s})$ on the field programmable gate array (FPGA) chip. Although such state-of-the-art RF SoCs support a maximum of only 16 channels, the bandwidth per DAC channel can be up to $3.25 \mathrm{GHz}$.

Multiplexing of multiple receiver channels into a single wideband signal with subsequent digitization using a high-precision RF-ADC is a trade-off between the number of ADCs $M$ and the signal bandwidth $B$ [114]. If the sample rate is $F_{s}$, then it follows that multiplexing in the primary Nyquist zone is bounded by $M B \leq$ $F_{s} / 2$. TDM, CDM [115-118] and FDM are the three main techniques for combining multiple analog streams into an over-sampled ADC with the objective of ADC reuse over multiple spatial channels. The multiplexing of $M$ antenna channels into a single ADC allows an $M$-fold increase in the supported independent spatial channels on a single RF-SoC device. 


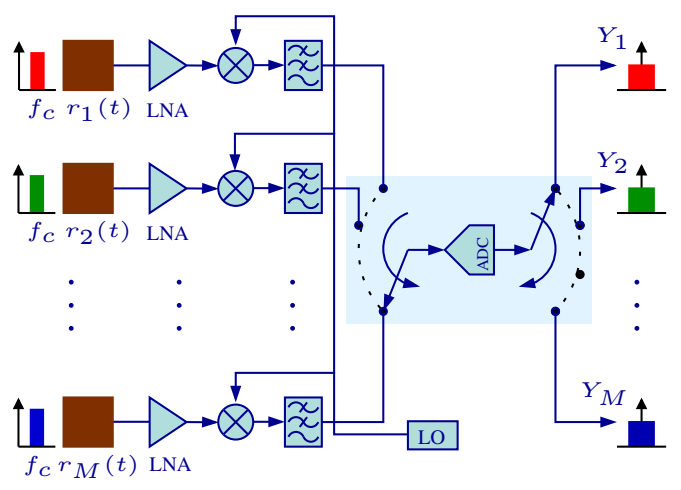

(a)

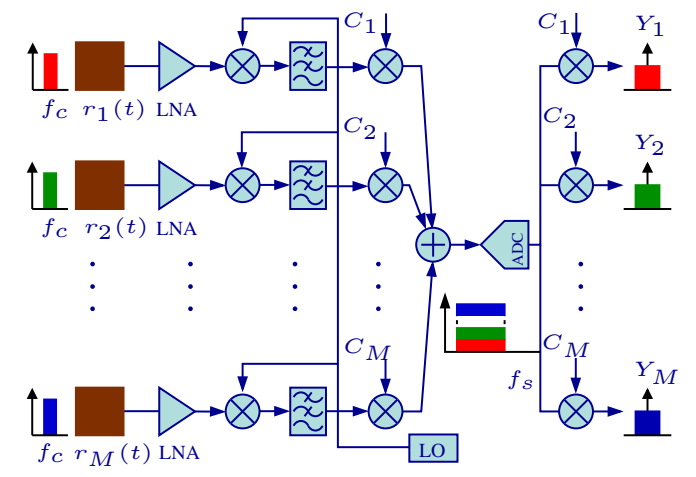

(b)

Figure 6.1: Implementation architectures for (a) TDM-based and (b) CDM-based hardware reduction schemes.

\section{TDM-based Receiver Approach}

In TDM, antenna elements are periodically switched to the same RF channel using a commutating analog switch as shown in Fig. 6.1 (a). The switching rate has to be greater than $M B \mathrm{~Hz}$ which, in turn, leads to artifacts and non-linearities in the signal due to practical constraints with real-world RF switches. Since the signal from each antenna is received for a duration of time of $1 / M$ duration, for an $M$ element system, this approach exhibits a considerable amount of SNR degradation. To overcome this, FDM-based and CDM-based approaches can be considered.

\section{CDM-based Receiver Approach}

CDM solves the problem of switching artifacts and non-linearities by modulating each channel using an orthogonal code (shown in Fig. 6.1 (b)), such as WalshHadamard (WH) codes [119], before summation and digitization in a single ADC $[115,116]$. The multiplexed channels are recovered using cross-correlation.

The use of an on-site coding receiver (OSCR) is a recently proposed approach [115-117] that facilitates the use of a single ADC instead of using a dedicated ADC 


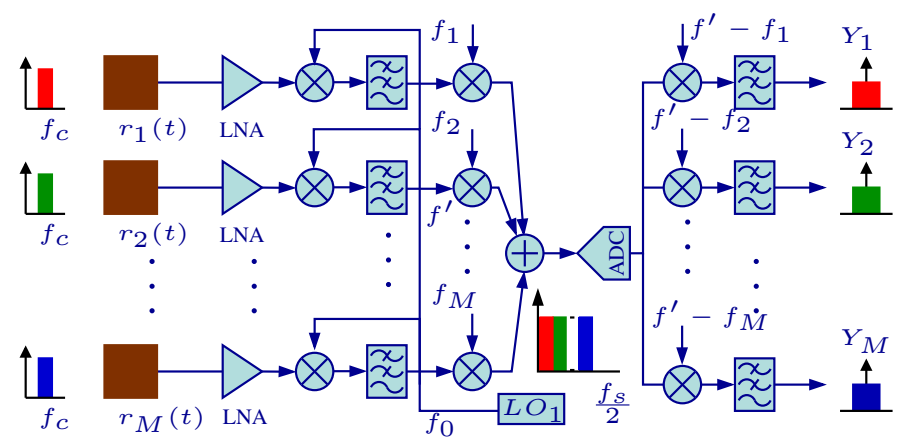

Figure 6.2: Implementation architecture for a FDM-based hardware reduction scheme.

for each antenna element. This approach uses the concept of CDM to uniquely identify the signal received by the corresponding antenna element, as shown in Fig. 6.1 (b). The receiver consists of a series of analog multipliers to code the output from each receiver by multiplying the output with a set of orthogonal, binary-coded (typically WH) waveforms. Since these coding waveforms are orthogonal, the coded outputs from multiple receivers can be summed without having interference, such that the summed output can be sampled and quantized using a single ADC. Once digitized, the original channels can be recovered with minimal signal degradation using cross-correlation in digital. In this approach, SNR does not degrade. Since every signal is coded with a unique code, this approach is resilient to interference and jamming. The combination of multiple $(M)$ channels require $M$ unique modulation waveforms for coded down-conversation and sampling. The bandwidth of these coded outputs increases extensively with the number of bits. Therefore, the ADCs are required have to high sampling rates and support high bandwidths in order for CDM to be applicable in wideband applications.

\section{FDM-based Receiver Approach}

In the FDM approach, signal received by each antenna are frequency-multiplexed into narrowband with different center frequencies. FDM-based approaches may use 
a single ADC to process each shared group of channels, but strict filtering is required to separate the channels in the digital domain. We propose FDM in multi-antenna access points with fully-digital DSP beamforming. The proposed concept is verified using a four-element prototype operating at $28 \mathrm{GHz}$ using Xilinx RF SoC ZCU1285 where a discrete Fourier transform (DFT) is applied spatially to achieve four orthogonal receive-mode $\mathrm{RFl}$ beams at $28 \mathrm{GHz}$, thereby experimentally verifying four-fold increase (i.e., $M=4$ ) in the number of supported spatial degrees of freedom per ADC channel.

\subsection{Design of the FDM Array Receiver}

\subsubsection{Multi-Stage Down-Conversion}

The proposed system shown in Fig. 6.2 will employ FDM for down-converting $M$ antenna signals into an intermediate frequency (IF) channel where each receiver is frequency translated to a known center frequency using a two-stage down-conversion. In Stage - 1, a common local oscillator (LO) is employed to achieve bulk downconversion to the microwave band $f_{0}$. Subsequently, in Stage -2 , an $M$-array of different LOs provide frequency-division multiplexed IF signals that are combined in the analog/microwave domain after suitable passive filtering to obtain the FDM signal that is sampled by a single ADC. After sampling, the IF signal is processed using DSP in which FDM-multiplexed signals are split into their corresponding antenna channels in baseband using digital sub-band filtering. Sub-band filtering is

realized in a real-time DSP filterbank, such as a polyphase finite impulse response (FIR) perfect-reconstruction filterbank [120]. Sub-banded channels are finally downconverted to baseband using one digital down-converter (DDC) per channel. 
Towards this goal, a variety of DSP filter topologies can be utilized for baseband signal recovery. The recovered signals correspond to the $M$ individual antenna channels and are available for subsequent multi-beam DSP beamforming, spatial interference nulling, computation of channel state information (CSI) or any other MIMO signal processing operation. Major challenges involved with FDM realization include the following:

1. Phase synchronization,

2. Computation of phase offset term corresponding to the element index,

3. Calibration of analog/digital Stage - 2 LO, and

4. Increased digital hardware complexity.

Section 6.1.2 presents an extensive analysis of the impact of the first three challenges and proposes an approach to overcome them. Digital hardware complexity reduction is achieved by designing optimized poly-phase FIR filter structures, which is discussed in Section 6.1.3.

\subsubsection{Phase Synchronization and Frequency Locking}

FDM across multiple antenna channels requires synchronization between the Stage2 analog down-conversion and the digital down-conversion to retrieve phase information from the $M$ channels. Here, we will discuss the mathematical reasoning behind this requirement. Consider a planar wave impinging on a $M$-element ULA with an inter-element spacing of $\Delta x$ at a direction of arrial (DOA) of $\psi$. The signal received by the $k^{t h}$ antenna element $r_{k}(t)$ is given by Eqn. (6.1), where $x(t)$ is the information-bearing signal.

$$
r_{k}(t)=x\left(t-\tau_{k}\right) \cos \left(\omega_{c} t-\theta_{k}\right)
$$


Here, $\omega_{c}=2 \pi f_{c}$ where $f_{c}$ is the carrier frequency and where $\theta_{k}=\omega_{c} \tau_{k}$ in which $\tau_{k}=\frac{\Delta x \sin \psi}{c}(k-1)$ and $c$ is the speed of light. Since $x\left(t-\tau_{k}\right) \approx x(t)$ for a narrowband signal, the signal $p_{k}(t), k \in[1,2, \ldots, M]$ at each of the $k^{t h}$ receiver after the first down conversion can be expressed as,

$$
\begin{aligned}
p_{k}(t) & =L P F\left\{r_{k}(t) \cos \left(\omega_{0} t+\phi_{0}\right)\right\} \\
& =K_{1} x(t) \cos \left[\left(\omega_{c}-\omega_{0}\right) t-\phi_{0}-\theta_{k}\right],
\end{aligned}
$$

where $K_{1}$ is a constant dependent on the gain of the low pass filter (LPF) response and $K_{1}=1 / 2$ for a unit gain filter. Here, $\omega_{0}=2 \pi f_{0}$ and the angle $\phi_{0}$ is the phase of the Stage-1 down-converter local oscillator (LO) at the initial time of consideration (i.e., $t=0)$. For $\left(\omega_{c}-\omega_{0}\right)=\omega^{\prime}$, Eqn. (6.2) is simplified as,

$$
p_{k}(t)=K_{1} x(t) \cos \left(\omega^{\prime} t-\theta_{k}-\phi_{0}\right)
$$

Assume there exists a temporal offset of $t_{k}^{\prime}$ between the Stage -2 down-converter and the digital down-conversion stage. This $t_{k}^{\prime}$ is caused by propagation delays and synchronization issues between the two systems. If the $k^{\text {th }}$ FDM output is $q_{k}(t)$ at the second stage of down-conversion, it is given by

$$
\begin{aligned}
q_{k}(t) & =\operatorname{LPF}\left\{p_{k}(t) \cos \left(\omega_{k}\left(t-t_{k}^{\prime}\right)\right)\right\} \\
& =\operatorname{LPF}\left\{p_{k}(t) \cos (\omega_{k} t-\underbrace{\left.2 \pi(k u+v) t_{k}^{\prime}\right)}_{k \alpha+\beta+\phi_{k}})\right\} .
\end{aligned}
$$

Since each $k^{\text {th }}$ frequency band in the FDM output has a unique center frequency, the discrete frequency variable $\omega_{k}$ can be expressed as $\omega_{k}=2 \pi(k u+v)$, where $(u, v) \in \mathbb{R}$. Since $t_{k}^{\prime}$ is a constant for a channel, we can find the angles $\alpha, \beta$, and $\phi_{k}$ such that, $2 \pi(k u+v)=k \alpha+\beta+\phi_{k}$, where $0 \leq\left(\alpha, \beta, \phi_{k}\right) \leq \pi$. The angle $\phi_{k}$ is caused by the mismatch of propagation delays and $\phi_{k}=0 ; k=[1,2, \ldots, M]$ for equal length paths of propagation. The term $k \alpha+\beta$ results from the lack of synchronization 
between the analog FDM LOs and the corresponding digital down-converter LOs. Therefore,

$$
\begin{aligned}
q_{k}(t) & =L P F\left\{K_{1} x(t) \cos \left(\omega^{\prime} t-\theta_{k}-\phi_{0}\right) \cos \left(\omega_{k} t-k \alpha-\beta-\phi_{k}\right)\right\} \\
& =K_{2} x(t) \cos (\left(\omega^{\prime}-\omega_{k}\right) t-\theta_{k}+\phi_{k}+k \alpha+\underbrace{\beta-\phi_{0}}_{\phi})
\end{aligned}
$$

Equation (6.6) shows that the phase shift has a linear dependency on the array index $k$, in addition to the phase shift caused by the inter-element propagation delay $\theta_{k}$, for an equal length (i.e., $\phi_{k}=0$ ) propagation. The digital beamforming core takes $\theta_{k}$ into account, and the $\phi$ parameter could be eliminated by calibration. Yet, the $k \alpha$ term still remains and causes an additional, progressive phase offset between each of the down-converted channels. Frequency mismatches and additional phase offsets have considerable effects in beamforming applications where the phase information has a vast importance. The use of frequency-locked oscillators allow the digital down-converter to bring the FDM channels down to the same frequency as the one required by the digital beamformer. In order for the proposed approach to be used in beamforming, it is required to eliminate the $k \alpha$ term from the equation. This can be achieved by the use of the same LO samples in the digital down-converter to generate the Stage - 2 LO signal to ensure the synchronization. This requires the operation of both the ADC and the DAC using the same clock. A calibration stage is required between the DAC oscillator and the digital down-converter to compensate for the phase offset term corresponding to the element index (i.e., the term $\left.\left(k \alpha+\phi_{1}+\phi_{2}\right)\right)$. The complex calibration coefficient $C_{k}=A_{k} e^{-j[\phi+k \alpha]}$ is determined by a test downconversion measurement at the broadside, and this value can be used to compensate for the issues caused by different cable lengths and RF components as shown in Fig. 6.4. Finding the $C_{k}$ values using an oscilloscope before sampling could be tricky, since all the $M$ inputs are combined at the ADC input. Therefore, $C_{k}$ needs to be 
set to $(1+0 j)$ for all $k$ in the initial design, and corresponding phase and magnitude offsets of the digitally down-converted (DDC) signals are measured at the broadside. To compensate for these magnitude mismatches and the phase offsets, calibration coefficients are updated, and the digital design is regenerated.

\subsubsection{DDC and FIR Filtering}

High sampling rates of ADCs (GSamples/s) can provide billions of data samples per second. However, the operation frequency of the digital hardware is limited due to certain timing restrictions as dictated by the critical path delay (CPD) in the design. Complex designs introduce larger CPDs to the system, which leads to a reduction of the operable clock frequency, and these designs often run at rates of few hundred MHz. Therefore, it is required to have a parallel processing system to process multiple samples at each digital hardware clock cycle. In practice, highspeed ADCs in an RF chain (shown in Fig. 6.3(a)) provide a polyphase data stream as shown in Fig. 6.3(b). In an $a$-phase system, the ADC provides $a$ samples at each digital hardware clock cycle. Therefore, it is required to follow a polyphase architecture when implementing the desired filter structures. The use of polyphase ADCs and the implementation of the FIR filterbank in polyphase result in lower clock rate requirement for sampling and DSP. The derivation of the polyphase filter function follows an approach similar to radix factorizing in discrete Fourier transform

implementations. Assuming that an $\mathrm{ADC}$ of $a$ channels with an $f_{s} / 2$ sampling clock is used and the $K^{\text {th }}$-order FIR filter with a $Z$ domain transfer function $H(z)$ is given by coefficients $b$ :

$$
y[n]=\sum_{k=0}^{K} b[k] x[n-k] .
$$



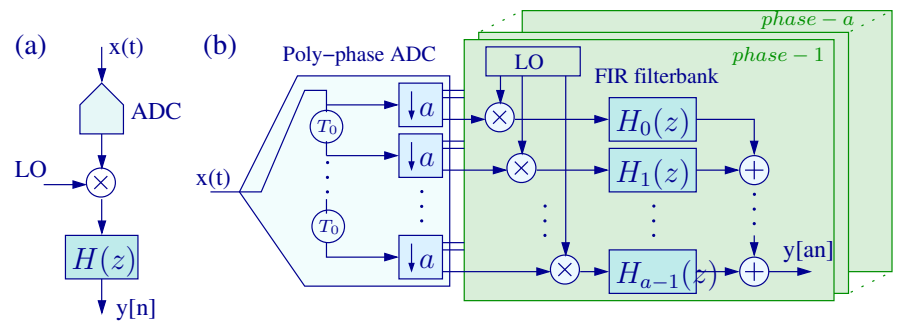

Figure 6.3: (a) Digital down-conversion. (b) Poly-phase filterbank.

By factorizing Eqn. (6.7), the polyphase filter functions for $a$ phases can be derived as:

$$
\begin{aligned}
y[n]=\overbrace{\sum_{k=0} b[a k] x[a(n-k)]}^{H_{0}}+\overbrace{\sum_{k=0}^{K / a} b[a k+1] x[a(n-k)+1]+\ldots}^{H_{1}}+\ldots \\
+\underbrace{\sum_{k=0}^{K / a} b[a k+a-1] x[a(n-k)+a-1]}_{H_{a-1}} .
\end{aligned}
$$

The filterbank can be implemented in polyphase as shown in Fig. 6.3(b). It should be noted that the output data stream from each phase is undersampled by a factor of $a$ and that the set of $a$ phases can reconstruct the total response by providing $a$ sets of outputs at each digital clock period. However, as the same filter is repeated $a$ times, the hardware complexity for polyphase structures increases by a factor of $a$. Designing the FIR filters in the digital domain and determining the guard band $\left(B_{g}\right)$ involve a trade-off between the system bandwidth and hardware complexity. We aim to pack as many frequency bands in the spectrum having the smallest possible guard band. FIR filter implementations to filter closely packed frequency bands require high-order "brick-wall filter" like structures. Polyphase implementations of FIR filters require extensive hardware resources. We employed 


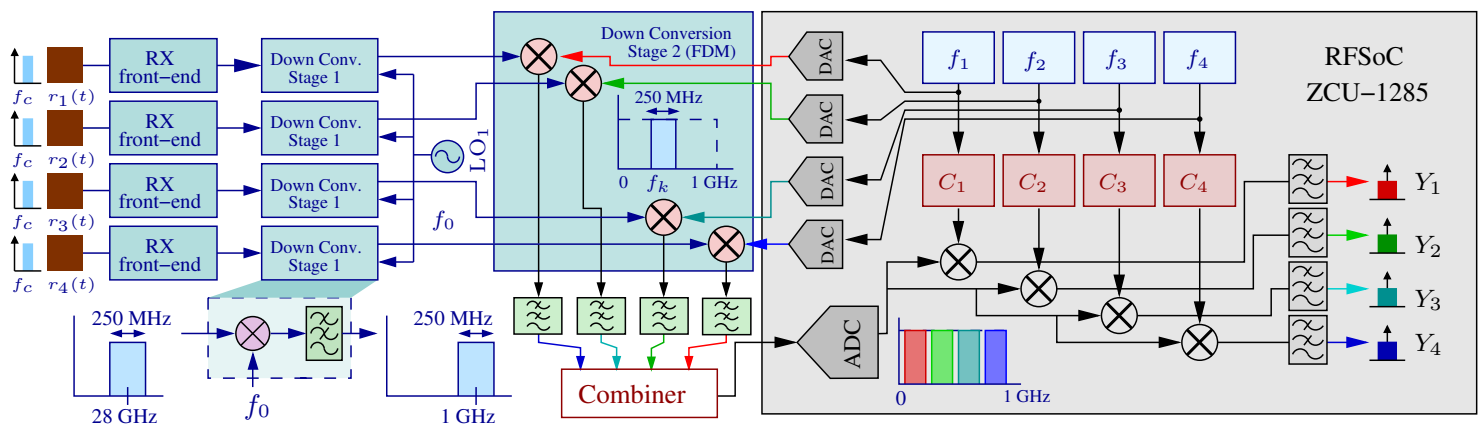

Figure 6.4: Overview of $F_{0}=28 \mathrm{GHz}, 240 \mathrm{MHz} /$ channel four-element FDM array receiver.

the Xilinx SSR blockset for Xilinx RF-SoCs. For synchronization, both the DAC and the ADC use a common clock signal.

\subsubsection{Test-Bed Validation}

A bank of $M$ phase- and frequency-locked oscillators is used to frequency translate inputs at known frequency offsets. The proposed FDM architecture is shown in Fig. 6.4. Here Stage -2 oscillators are required to be synchronized with each other as well as with the digital down-conversion clock, using a stable low-jitter reference clock via a bank of $N$ frequency synthesizers (where $N$ is an integer). To achieve precisely synchronized samples, we use an RF-SoC DAC to generate $\mathrm{LO}_{2}$ while using the same sampling clock for both the ADC and the DAC. A double-sided signal bandwidth of $B=240 \mathrm{MHz}$ per receiver and $M=4$ antenna elements, each designed for $28 \mathrm{GHz}$ operation, have been assumed. The double-sided bandwidth of four frequency multiplexed channels with a $10-\mathrm{MHz}$ guard band $\left(B_{g}\right)$ per channel is $1 \mathrm{GHz}$.

In the next stage $(S t a g e-2)$, the IF signals are down-converted to the required IF for sampling and centered at $L O_{2, k}=250 k-125 \mathrm{MHz}$, where $k=1,2 \ldots M$. LO frequencies of $125,375,625$, and $875 \mathrm{MHz}$ are then applied to each of the 

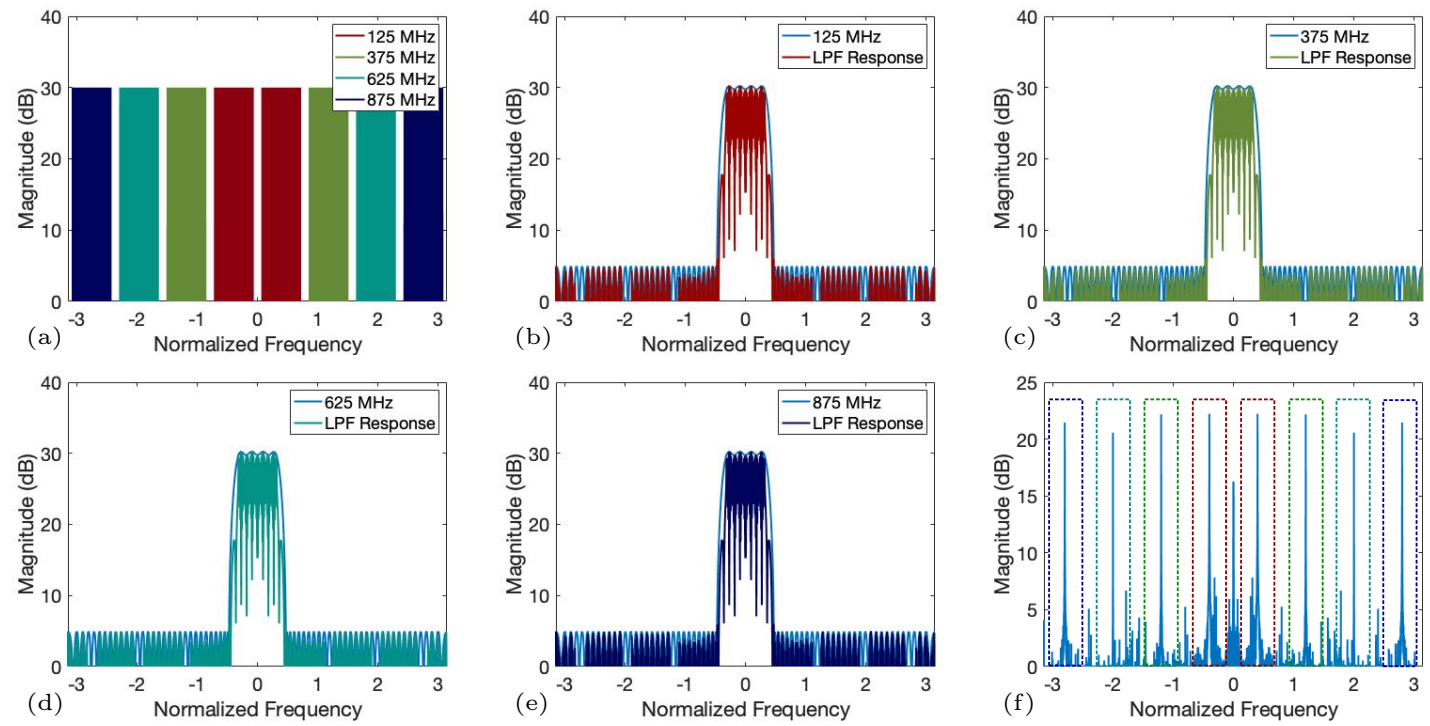

Figure 6.5: (a) Simulation results showing the combined output from the Stage - 2 analog down-conversion fed to the ADC. Digital down-conversion and filtering for narrowband signals centered at (b) $125 \mathrm{MHz}$, (c) $375 \mathrm{MHz}$, (d) $625 \mathrm{MHz}$, and (e) $875 \mathrm{MHz}$. (f) Measurements of the combined signal sampled by RFSoC.

antennas to translate the received signals to 875-, 625-, 375-, and 125-MHz center frequencies, respectively. The IF components are fed into a combiner to create the FDM signal. Note that the FDM "baseband" can be sampled using a single ADC, and the subsequent digital signal can be filtered, down-converted, and subjected to a Hilbert transform to obtain the quadrature component. The inter-band frequency guard bands $B_{g}$ are needed to accommodate finite-order FIR filtering in the digital domain. The output from this second-stage analog down-conversion is fed to the $\mathrm{ADC}$ and is sampled at a sampling rate of $F_{s}=2 \mathrm{GHz}$.

Figure 6.5 (a) shows the sampled spectrum for $M=4$ FDM channels, where each has a bandwidth of $250 \mathrm{MHz}$ (i.e., including $B_{g}$ ). Simulations have used a combination of 32 tones with a space of $240 / 32=7.5 \mathrm{MHz}$ to generate the wideband signal, such that $\sum_{n=1}^{16} \cos \left(2 \pi\left(f_{k} \pm 7.5 n\right) t\right)$ for $k=1,2,3,4$. The digital downconverter causes the aliased image components to fall back into the same Nyquist 

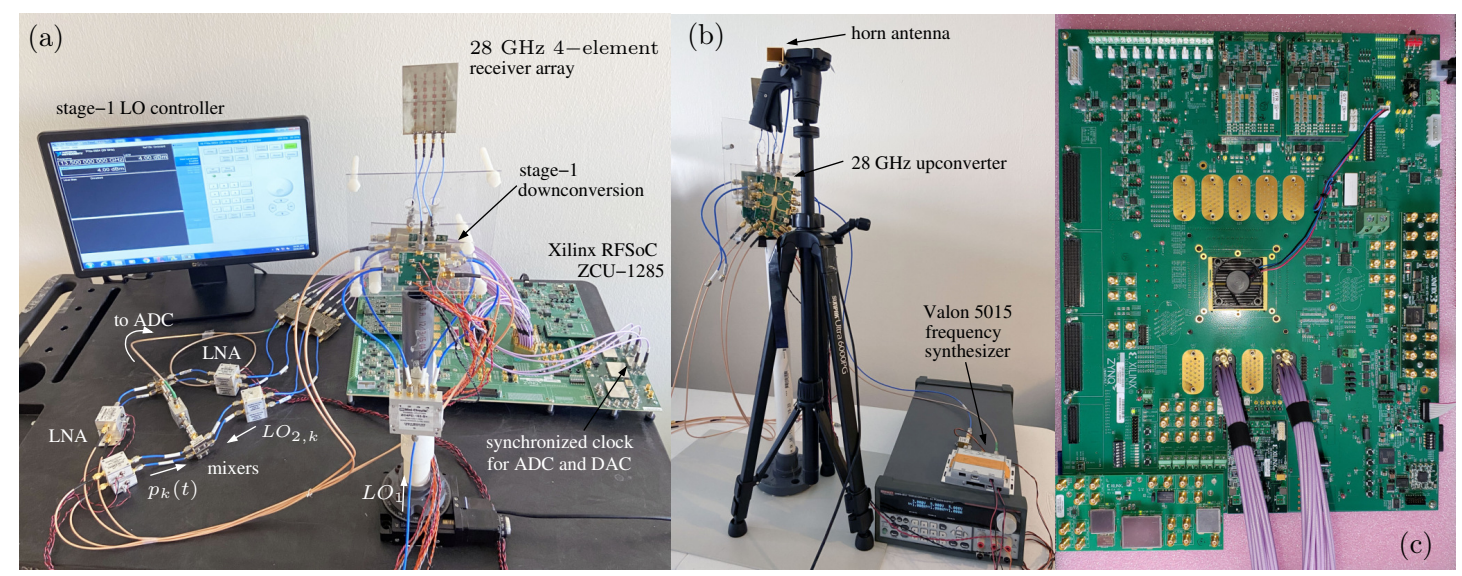

Figure 6.6: Experimental setup showing (a) the four-element, 28-GHz receiver antenna array and (b) the transmitter array, and (c) Xilinx RFSoC ZCU1285.

zone. Highly selective FIR low-pass digital filters of the order of 70 were applied to filter out the image components from each of the antenna spectra. The filtered spectrum from the simulation of the DDC is shown in Figs. 6.5 (b-e).

\subsubsection{Digital FDM Multi-Beam Measurements}

The proposed digital FDM concept is verified through the measurements obtained by using the prototype experimental setup shown in Fig. 6.6. A four-element patch antenna array operating in the frequency range of 27.5 to $28.35 \mathrm{GHz}$ that was designed in our previous study [121] was used for this purpose. The Stage - 1 down conversion for each of the 28-GHz receiver antennas use an Analog Devices EVAL01-HMC1065LP4E [122] module that contains HMC1065 chips. The experimental validation requires an initial measurement to calibrate the phases of each of the RF channels as well as synchronize $f_{k}$ with DDC LO. Therefore, a pilot tone is transmitted at $28 \mathrm{GHz}$, and the antenna array is used to receive the signal at a DOA of $0^{\circ}$ as measured from the broadside. 


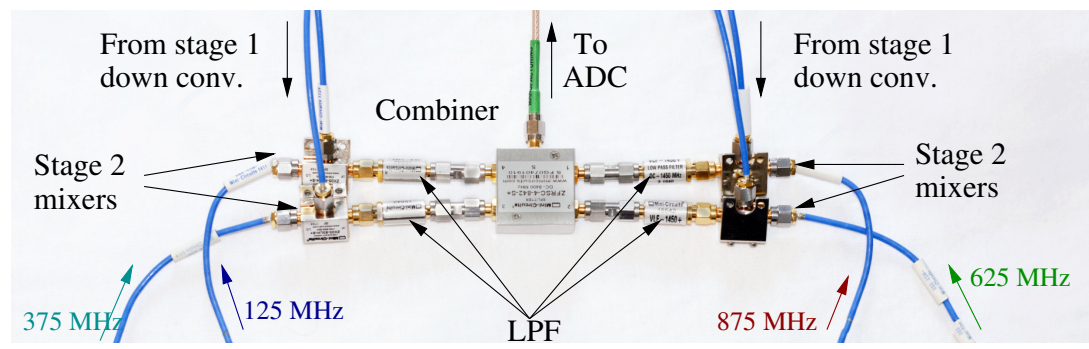

Figure 6.7: Down conversion Stage - 2 setup for 4 element, $28 \mathrm{GHz}$ antenna array.
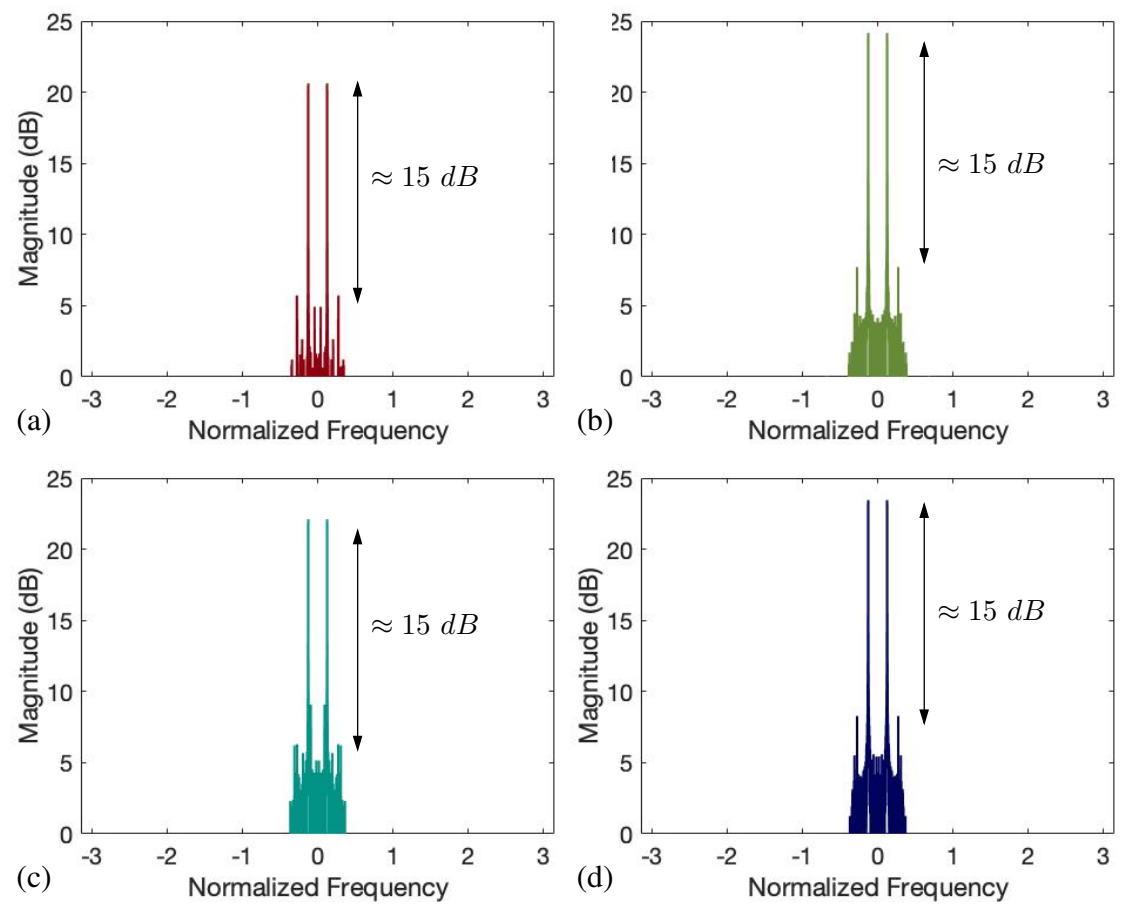

Figure 6.8: Retrieved carrier signals digitally down-converted to baseband.

Each EVAL01-HMC1065LP4E RF receiver contains an internal frequency doubling circuit in the LO path of the first down-conversion stage. A National Instruments RF signal generator was used to generate the first LO signal of $13.5 \mathrm{GHz}$, yielding an $\mathrm{LO}$ of $27 \mathrm{GHz}$ after the frequency doubler. The 1-GHz centered IF signals resulted from mix-down operation are low-pass-filtered for image-rejection and noise suppression, and are passed to the second down-conversion stage as shown in Fig. 6.7. 

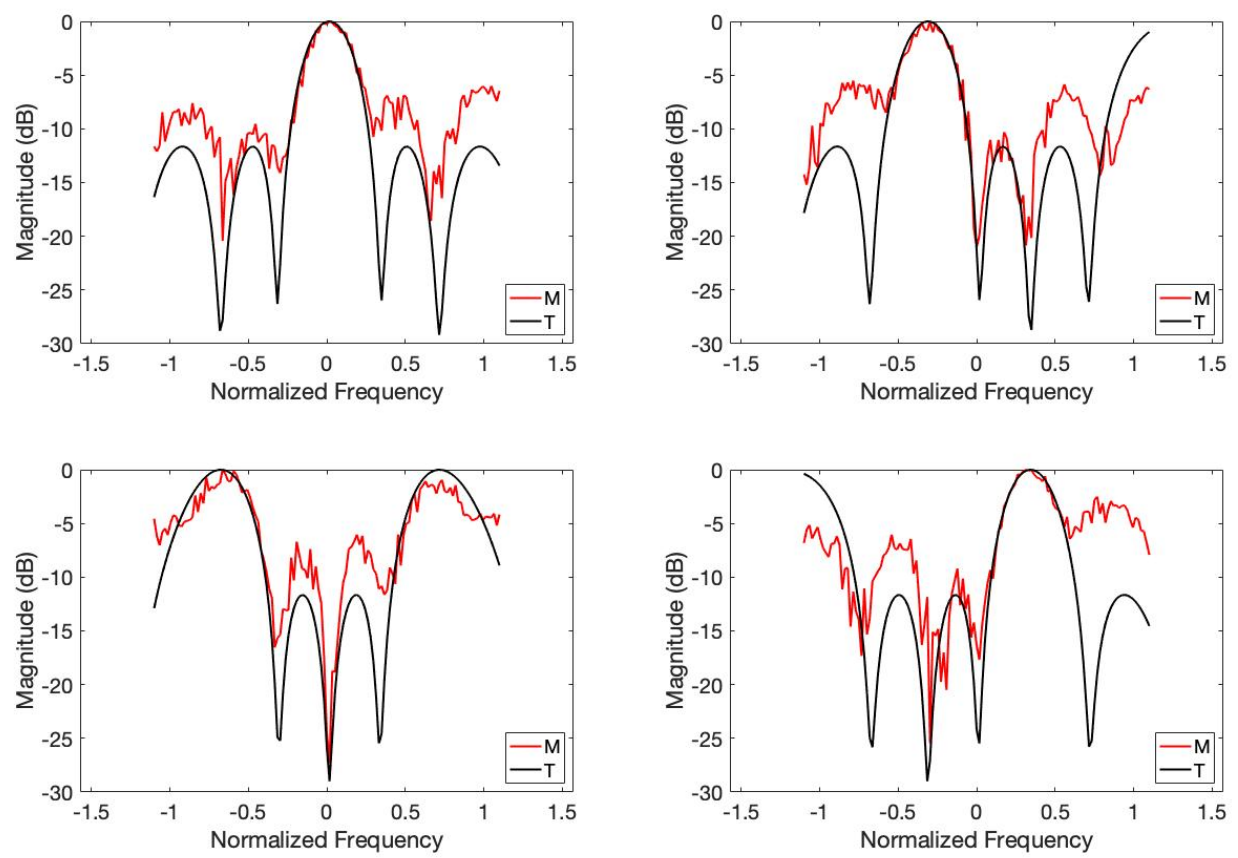

Figure 6.9: Theoretical and measured beams at $28 \mathrm{GHz}$ using digital real-time beamforming using a single ADC to sample 4 independent channels.

RF-SoC DACs were used to generate the four phase- and frequency-synchronized LO frequencies that are needed for the Stage -2 down-conversion. Frequency translation at the second stage is used to down-convert the antenna outputs to different center frequencies such that the corresponding narrowband signals from the four antennas are visible at $125 \mathrm{MHz}, 375 \mathrm{MHz}, 625 \mathrm{MHz}$, and $875 \mathrm{MHz}$. Four commercial-off-the-shelf (COTS) RF mixers (MiniCircuits ZX05-12MH-S+) were used in Stage - 2 followed by VLF-180, VLF-400+, VLF-630+, and VLF-800 LPFs respectively. These LPFs were chosen to have sufficient attenuation to suppress the effect of the second-order-harmonic of the corresponding FDM channel. A COTS combiner (MiniCircuits ZFRSC-4-842-S+) was used to combine the four RF channels that were frequency translated by FDM. 
After the Stage - 2 down-conversion, outputs are combined and are sampled at $2 \mathrm{GS} / \mathrm{s}$ to obtain the spectrum shown in Fig. 6.5 (f). The Xilinx ZCU-1285 FPGA shown in Fig 6.6 (c) is used to sample the input, DDC, and FIR filtering. Since the digital hardware is designed to run using a $250-\mathrm{MHz}$ clock, samples at $2 \mathrm{GS} / \mathrm{s}$ are processed using an eight-phase multi-rate DSP implementation (i.e., $a=8$ ). Samples arriving from the ADC are then digitally down-converted to a lower IF (10 MHz in this case) and then low-pass-filtered to retrieve the spectra. Down-conversion plots shown in Fig. 6.8 presents information on the frequency and the magnitude of the down-converted signals. Measurements show an SINR of approximately $15 \mathrm{~dB}$, which is an artifact of the measurement setup and LPF gains.

A digital spatial FFT was used to generate multiple beams. The RF source DOA was swept from $-\pi / 3 \leq \psi \leq \pi / 3$ (measured from the broadside) to obtain spatial beam patterns. MATLAB was used to generate the theoretical beam pattern for a four-element antenna array with $\Delta x=0.75 \lambda$ spacing between antenna elements, and the measurements of the received RF beams are plotted with their corresponding theoretical beams in Fig. 6.9. An ideal digital beamformer is expected to have the beam pattern shown in black, whereas the measured beam pattern (shown in red) is observed to show the desired array factor with some deviations in the sidelobes. The 4-beam beamformer is operating as expected but there is deviation in the sidelobes, most likely due to reflections in the measurement area (we were unable to access a sufficiently large anenoic chamber due to pandemic restrictions over the last six months; therefore the measurements were conducted in an indoor open space that may suffer from unexpected reflections).

The same mixer was used in all four RF channels in the Stage -2 downconversion, and the LPFs were chosen carefully to reduce the effect of second- and third-order harmonics, which is the primary determinant of the SINR in this system. 
Second-order harmonics for $125 \mathrm{MHz}$ and $250 \mathrm{MHz}$ channels lie closer to the passband and the filter roll-offs of the chosen COTS are not sufficient to completely block the prominence of intermods and harmonics. In addition, implementation of two cascaded down-conversion stages may combine the noise figures of active and passive elements in both stages, leading to a lower SINR. Effects of such non-idealities can be reduced by choosing steeper LPFs at a tradeoff with the cost. Extension of the implemented system to larger values of $M$, say $M=16$, could achieve greater savings from a cost standpoint for implementing massive MIMO at scale for large arrays.

\subsection{Conclusion}

The requirement for large numbers of independent ADC channels is a bottleneck for implementation of mm-wave massive MIMO. The largest available RF SoC device can accommodate 16 ADCs per chip. FDM was proposed at the receiver to reduce ADC counts in order to facilitate large massive-MIMO arrays having multiples of 16 independent receivers without compromising the spatial degrees of freedom using available devices. Multiple antenna signals were multiplexed in the frequency domain and were sampled using a single ADC to increase the number of antennas per RF SoC by a factor of $M$. The proposed architecture was verified for a four-element 28-GHz digital array with fully digital beamforming using a Xilinx RF SoC ZCU-1285. The proposed architecture allows over-sampled ADCs to digitize $M$ independent receive antennas via FDM. Measurements of the four-element 28-GHz system shows support for $240 \mathrm{MHz}$ of bandwidth per channel at an SINR of $15 \mathrm{~dB}$. Improvements in RF circuit designs (better linearity and noise reduction) will enable future systems with improved SINR. A multi-beam digital beamformer was realized 
using four-point FFTs in the digital domain using the four-antenna outputs sampled using a single ADC. The proposed method allows 64 antennas and 64 independent spatial channels at $28 \mathrm{GHz}$ when using a Xilinx ZCU-1285 RF SoC with 16 ADCs. 


\section{CHAPTER 7}

\section{USE OF ADFT IN DIGITAL BEAMFORMING TO REDUCE HARDWARE COMPLEXITY}

Methods discussed in previous chapters are used in reduction of ADC complexity in RF circuits. Processing of received signals is mostly happened in the digital domain using digital hardware. Digital FFT cores have numerous applications in multidimensional systems, where lower complexities are preferred to achieve lower area and power consumption. This chapter discusses about the digital implementation of a low complexity 1024-point ADFT core to replace FFT and its applications.

The DFT is a linear transform widely applied to convert a sampled (discretetime) signal into a representation over the discrete frequency domain. The $N$ point DFT computes $N$ uniformly spaced frequency domain outputs (also called "bins") using $N$ uniformly sampled discrete-time signal values by means of an $N \times N$ transformation matrix. In a typical scenario, both spatial and temporal signals can be transformed to the corresponding frequency domain using the DFT [123]. However, the computational complexity of computing the $N$-point DFT using direct matrix-vector multiplication is $\mathcal{O}\left(N^{2}\right)$ where $\mathcal{O}(\cdot)$ represents the big O notation for asymptotic complexity [124].

The computational complexity of computing the $N$-point DFT can be significantly reduced by using a suite of fast algorithms known as FFTs which can reduce the computational complexity to $\mathcal{O}\left(N \log _{2} N\right)$. Because implementations of the

multiplication operation generally require much more chip area and/or processing time than other arithmetic and logic operations, the computational complexity of computing the DFT is usually expressed in terms of its multiplication count [125].

The required number of multiplications depends on the fast algorithm employed for the particular transform length $N$ in consideration. In [87], the theoretical 
lower bound for the DFT multiplicative complexity was established as a function of $N$. The search for particular $N$-point FFT methods that strives to attain the theoretical lower bound for multiplicative complexity is a separate field of research in signal processing, computer science, and applied mathematics, with a multitude of FFT algorithms available [126-129].

Usually, FFTs are based on sparse factorizations of the DFT matrix and provide DFT computations at arbitrarily high levels of precision depending on a particular implementation. For example, a typical double precision realization in software using a modern 64-bit processor can provide nearly-exact computation. However, these realizations are often prohibitively slow. For example, in modern MRI where hundreds or thousands of antenna elements are used, computational efficiency is vital for real-time imaging of fast-moving objects (e.g., arterial blood flow or heart musculature), and may require MIMO antenna signal processing and/or exploitation of sparsity [130,131].

Fortunately, such high accuracy is of limited practical relevance in certain RF applications [16], such as MRI, SDR, and radar signal processing, where the accuracy of the results is limited by other system parameters or environmental conditions (e.g., thermal noise in a receiver, or harmonic distortion in a microwave mixer or amplifier). In such applications, relentless pursuit of high accuracy in the computation of the DFT is fruitless in terms of overall performance, and smart system design recognizes this for power and cost optimization. A good example of this was the design of the GSM second-generation wireless cellphone standard, and its choice of Gaussian Minimum Shift Keying (GMSK) modulation. Designers realized that the wireless system noise due to oscillators and channel multipath effects was much greater than the self-noise induced by intersybmol interference (ISI) of the GMSK modulation. Choosing a non-coherent modulation scheme such as GMSK would not 
be ideal in a perfect channel, but was suitable in this case due to practical device and channel limitations (e.g., self-noise was well below the system-level noise floor).

However, the DFT computations should not be too coarse, since signal errors and uncertainties imposed by the system become dominant only after a certain level of precision is achieved. In other words, the precision of the DFT computation can only be compromised as long as the overall performance of the system is negligibly affected.

The adoption of ADFT computation opens up new possibilities for fast algorithms which do not compute the DFT in a strict mathematical sense, but nevertheless can be good enough for a particular application. Such approximations allow tremendous reductions in computational complexity; much more than the reduced complexity levels of traditional FFTs, albeit at a deterministic price paid in performance due to inherent non-exact computation. We suggest that such a loss of accuracy is justified when one considers the significant reductions in chip-area and power consumption of the approximate algorithms compared to traditional FFT realizations. The loss of accuracy is unlikely to have significant impacts on applications such as digital RF beamforming which has performance dominated by other factors, such as the mutual coupling between elements, mismatches of components, and non-linearities in the RF receiver electronics. In this chapter, we explore three fast algorithms for the approximate computation of the 1024-point DFT. The introduced 1024-point ADFT is based on a recently proposed 32-point DFT approximation and multiplierless fast algorithm $[132,133]$ which furnishes a reasonable' approximation of the 32-point DFT albeit without using any multiplications at all (i.e., it is an adder-only signal flow graph).

The proposed work extends the 32-point ADFT $[132,133]$ to the 1024-point transform case via three new fast algorithms having different trade-offs in computa- 
tional complexity and computational accuracy compared to the baseline exact DFT. The proposed approximations are compared to the DFT in terms of arithmetic complexity and relevant beamforming metrics, such as worst side lobe. The resulting hardware designs are compared in terms of hardware resource consumption metrics such as area, maximum operating frequency, critical path delay, and dynamic power. 32-point ADFT matrix derived by Dr. Renato Cintra and his team was used in deriving an ADFT matrix for 1024-point case.

The chapter is organized as follows. Section 7.1 reviews the DFT and selected popular FFT algorithms. In Section 7.2, we discuss the mathematical background for the 32-point DFT approximation introduced in [132] and describe its associated fast algorithm in matrix form. In Section 7.3, we present 1024-point DFT approximations and discuss three different algorithms to implement them. Section 7.4 explores the digital VLSI realization of the proposed 1024-point DFT approximation. Section 7.5 discusses an application of the proposed transform in the context of wireless modulation.

\subsection{Review of the DFT and FFT}

In this section, we cover mathematical background related to the DFT definition and FFT algorithms.

\subsubsection{DFT Mathematical Definition}

Let the vector $\mathbf{x}=\left[\begin{array}{llll}x[0] & x[1] & \ldots & x[N-1]\end{array}\right]^{\top}$ represent a signal with $N$ samples. The DFT maps the input signal $\mathbf{x}$ into an output signal 


$$
\begin{gathered}
\mathbf{X}=\left[\begin{array}{llll}
X[0] & X[1] & \cdots & X[N-1]
\end{array}\right]^{\top} \text { according to the following relationship: } \\
X[k] \triangleq \frac{1}{\sqrt{N}} \sum_{n=0}^{N-1} x[n] \cdot \omega_{N}^{n k}, \quad k=0,1, \ldots, N-1,
\end{gathered}
$$

where $\omega_{N}=e^{-j \frac{2 \pi}{N}}$ is the $N$ th root of unity and $j \triangleq \sqrt{-1}$. On the other hand, the inverse DFT (IDFT) is given as

$$
x[n]=\frac{1}{\sqrt{N}} \sum_{k=0}^{N-1} X[k] \cdot \omega_{N}^{-n k}, \quad n=0,1, \ldots, N-1 .
$$

The DFT of $\mathbf{x}$ can be expressed through a matrix-vector multiplication $\mathbf{X}=\mathbf{F}_{N} \cdot \mathbf{x}$, where

$$
\mathbf{F}_{N}=\frac{1}{\sqrt{N}}\left[\begin{array}{ccccc}
1 & 1 & 1 & \ldots & 1 \\
1 & \omega_{N} & \omega_{N}^{2} & \ldots & \omega_{N}^{(N-1)} \\
1 & \omega_{N}^{2} & \omega_{N}^{4} & \ldots & \omega_{N}^{2(N-1)} \\
1 & \omega_{N}^{3} & \omega_{N}^{6} & \ldots & \omega_{N}^{3(N-1)} \\
\vdots & \vdots & \vdots & \ddots & \vdots \\
1 & \omega_{N}^{(N-1)} & \omega_{N}^{2(N-1)} & \ldots & \omega_{N}^{(N-1)(N-1)}
\end{array}\right]
$$

is the $N$-point DFT matrix [134].

\subsubsection{FFT Algorithms}

The computational complexity associated with performing the $N$-point DFT operation in direct form is $\mathcal{O}\left(N^{2}\right)$. This complexity is prohibitive for most engineering applications since a high number of operations accounts for (i) higher energy consumption; (ii) higher latency; (iii) higher number of gates; and, in consequence, (iv) higher chance of system failure. To address these issues, FFT factorizations furnish a product of sparse (mostly zeros) matrices that reduces the DFT computational complexity to $\mathcal{O}(N \log N)$. Different FFT algorithms can be identified in the literature [135-138]. In particular, we separate the Cooley-Tukey FFT [125], the split-radix FFT [136], and the Winograd FFT [139], which are given the following brief descriptions. 


\section{Cooley-Tukey Algorithm}

A very popular form of the Cooley-Tukey algorithm is the radix-2 decimation-intime FFT, which splits the $N$-point DFT computation into two $N / 2$-point DFT computations resulting in an overall reduced complexity [135]. Recursive use of this algorithm reduces the number of multiplications from $\mathcal{O}\left(N^{2}\right)$ down to $\mathcal{O}\left(N \log _{2} N\right)$.

\section{Split-radix Algorithm}

This is a variant of the Cooley-Tukey FFT algorithm which uses a blend of radix-2 and radix-4 by recursively expressing the $N$-point DFT in terms of one $N / 2$-point DFT and two N/4-point DFT instantiations [136]. The split-radix algorithm can reduce the overall number of additions required to compute the DFT of size power of two without increasing the number of multiplications [140].

\section{Winograd Algorithm}

The Winograd algorithm exploits the multiplicative structure on the data indexing of DFT and converts it into a cyclic convolution computation $[137,138]$. In several particular cases, the Winograd algorithm achieves the theoretical minimum multiplicative complexity [87] as shown in [137]. For large DFT blocklengths that can be decomposed as a product of small primes, Winograd algorithm achieves near linear complexity [125].

\subsubsection{Matrix Representation of the $N^{2}$-point DFT in Terms of the $N$-point DFT}

We derive a matrix representation for the computation of the $N^{2}$-point DFT in terms of the $N$-point DFT via a radix- $N$ FFT approach. Generally speaking, the 
$N^{2}$-point DFT computation corresponds to a vector-matrix multiplication with a $N^{2} \times N^{2}$ matrix transformation:

$$
\mathbf{X}=\mathbf{F}_{N^{2}} \cdot \mathbf{x}
$$

The above computation can be rewritten by directly invoking the Cooley-Tukey algorithm in its more general form as detailed in [125, p. 69]. By explicitly following the Cooley-Tukey algorithm, the $N^{2}$-point DFT can be computed by means of:

1. address-shuffling the input column vector into a 2D $N \times N$ array;

2. computing the $N$-point DFT of each array column using FFTs;

3. element-wise multiplying the twiddle-factors;

4. computing the $N$-point DFT of each resulting row using FFTs; and

5. undoing the address shuffling to convert the obtained $2 \mathrm{D}$ array into the final output column vector.

The $1 \mathrm{D}$ to $2 \mathrm{D}$ mapping can be accomplished by means of the inverse vectorization operator invvec(·) [141] (Cf. $[142,143])$ which obeys the following mapping:

$$
\operatorname{invvec}\left(\left[\begin{array}{c}
x_{0} \\
x_{1} \\
\vdots \\
x_{N^{2}}
\end{array}\right]\right)=\left[\begin{array}{cccc}
x_{0} & x_{N} & \cdots & x_{N(N-1)} \\
x_{1} & x_{N+1} & \cdots & x_{N(N-1)+1} \\
\vdots & \vdots & \ddots & \vdots \\
x_{N-1} & x_{2 N-1} & \cdots & x_{N^{2}-1}
\end{array}\right] .
$$

From the above discussion, we have that the $N^{2}$-point DFT shown in (7.4) can be given the following matrix expression based on the Cooley-Tukey algorithm:

$$
\mathbf{X}=\operatorname{vec}\left(\left\{\mathbf{F}_{N} \cdot\left[\boldsymbol{\Omega}_{N} \circ\left(\mathbf{F}_{N} \cdot(\operatorname{invvec}(\mathbf{x}))^{\top}\right)\right]^{\top}\right\}^{\top}\right)
$$


where $\operatorname{vec}(\cdot)$ is the matrix vectorization operator $[144$, p. 239], o is the Hadamard element-wise multiplication [144, p. 251], the superscript $\top$ denotes simple transposition (non Hermitian), and $\boldsymbol{\Omega}_{N}$ is the twiddle-factor matrix given by $\boldsymbol{\Omega}_{N}=$ $\left(\omega_{N^{2}}^{m \cdot n}\right)_{m, n=0,1, \ldots, N}$. Noting that $\boldsymbol{\Omega}_{N}^{\top}=\Omega_{N},(7.6)$ can be further simplified. In particular, for $N=1024=32^{2}$, we have

$$
\mathbf{X}=\operatorname{vec}\left(\left[\boldsymbol{\Omega}_{32} \circ\left(\mathbf{F}_{32} \cdot(\operatorname{invvec}(\mathbf{x}))^{\top}\right)\right] \cdot \mathbf{F}_{32}^{\top}\right)
$$

The inner DFT call corresponds to row-wise transformation of invvec(x), whereas the outer DFT performs column-wise transformations on the resulting intermediate computation. The formulation shown in (7.7) is the fundamental expression on which the proposed approximations in this work are based.

\subsection{Multiplierless 32-point ADFT}

In this section, the adopted multiplierless 32-point ADFT, introduced in [132], is presented and its complexity and error analysis are discussed.

\subsubsection{Matrix Representation}

The considered 32-point ADFT matrix — denoted by $\hat{\mathbf{F}}_{32}$ - can be computed through a product of sparse matrices whose real and imaginary parts of its coefficients contains only \pm 1 entries. Such simple arithmetic leads to hardware designs that can be realized with adders only. 
To present the factorization of $\hat{\mathbf{F}}_{32}$, we need the auxiliary structures shown next. Let $\mathbf{B}_{t}$ be a $t \times t$ real matrix given by

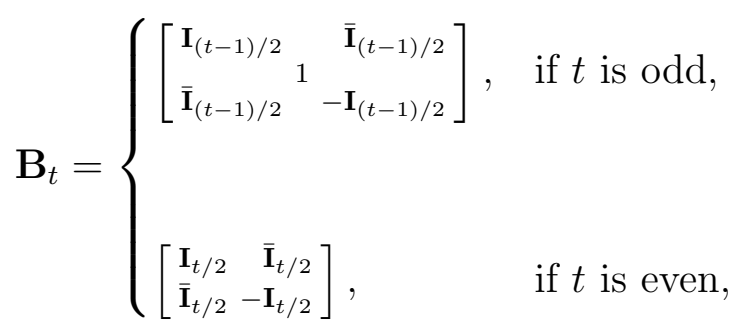

where $\mathbf{I}_{k}$ and $\overline{\mathbf{I}}_{k}$ being the identity and counter-identity matrix of order $k$, respectively. Let also $\mathbf{Z}_{1}, \mathbf{Z}_{2}$, and $\mathbf{Z}_{3}$ be the following matrices (for clarity, only the non-zero elements are shown):

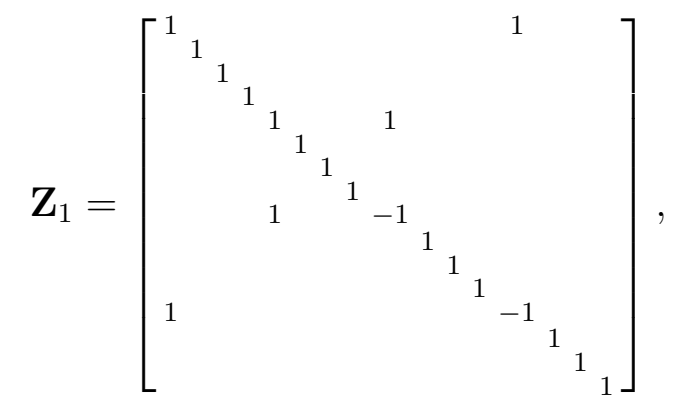

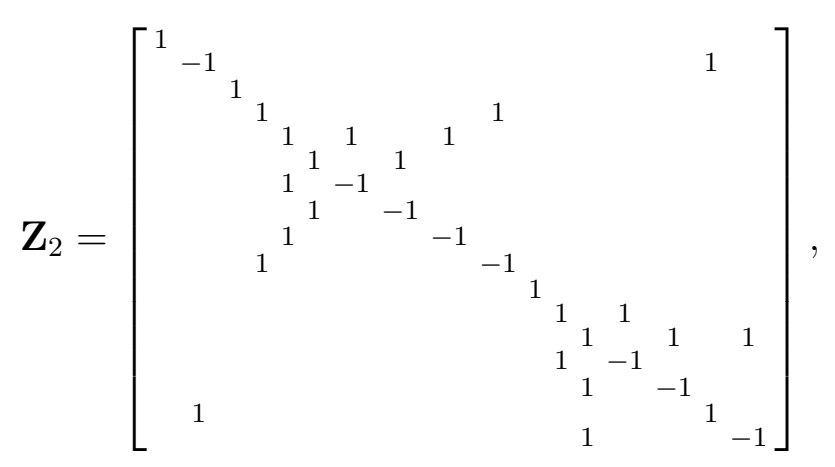

and

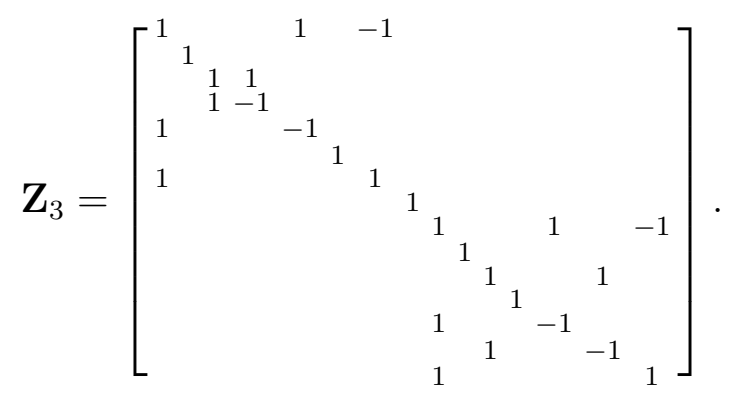


The 32-point ADFT matrix is factorized into eight sparse matrices $\mathbf{W}_{k}$, for $k=$ $0,1, \ldots, 7$, according to $[133]$

$$
\hat{\mathbf{F}}_{32}=\mathbf{W}_{7} \cdot \mathbf{W}_{6} \cdot \mathbf{W}_{5} \cdot \mathbf{W}_{4} \cdot \mathbf{W}_{3} \cdot \mathbf{W}_{2} \cdot \mathbf{W}_{1} \cdot \mathbf{W}_{0},
$$

where

$$
\begin{aligned}
& \mathbf{W}_{0}=\left[\begin{array}{ll}
{ }^{\mathbf{B}_{17}} & \\
& \mathbf{B}_{15}
\end{array}\right], \quad \mathbf{W}_{1}=\left[\begin{array}{cc}
\mathbf{I}_{16} & {\left[\begin{array}{cc}
{ }^{0} & \\
\mathbf{I}_{15}
\end{array}\right]} \\
{\left[\begin{array}{l}
0 \\
{ }^{0} \mathbf{I}_{15}
\end{array}\right]} & \mathbf{I}_{16}
\end{array}\right], \\
& \mathbf{W}_{2}=\left[\begin{array}{lllll}
\mathbf{B}_{9} & & & \\
& \mathbf{B}_{7} & \\
& & \mathbf{I}_{16}
\end{array}\right], \quad \mathbf{W}_{3}=\left[\begin{array}{llllll}
\mathbf{B}_{5} & & & & & \\
& & & & & \\
& & \mathbf{B}_{3} & & & \\
& & & & & \\
& & & \mathbf{B}_{3} & & \\
& & & \mathbf{B}_{3} & \\
& & & & \mathbf{Z}_{1}
\end{array}\right], \\
& \mathbf{W}_{4}=\left[\begin{array}{lllll}
\mathbf{B}_{3} & & & & \\
& \mathbf{B}_{2} & & & \\
& & \mathbf{B}_{4} & & \\
& & \mathbf{B}_{4} & \\
& & & \mathbf{B}_{2} & \\
& & & \mathbf{Z}_{2}
\end{array}\right], \quad \mathbf{W}_{5}=\left[\begin{array}{llll}
\mathbf{B}_{2} & & \\
& \mathbf{I}_{15} & \\
& & \mathbf{Z}_{3}
\end{array}\right],
\end{aligned}
$$

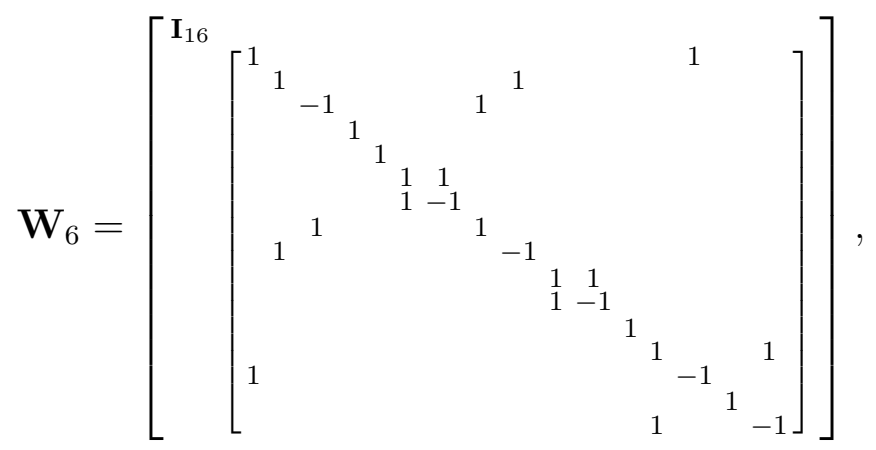

and $\mathbf{W}_{7}$ is given in (7.13).

\subsubsection{Arithmetic Complexity}

The associated multiplicative complexity of the Cintra-32-point ADFT is null [133]. No bit-shifting operations are required. The only source of arithmetic complexity is the number of additions in the factorization in (7.12). Considering the case of 
complex input, the matrices $\mathbf{W}_{0}, \mathbf{W}_{1}$, and $\mathbf{W}_{4}$ require 60 real additions each and no bit-shifting. The matrices $\mathbf{W}_{2}, \mathbf{W}_{3}$, and $\mathbf{W}_{5}$ require 28 real additions each and no bit-shifting, as well. The matrix $\mathbf{W}_{6}$ requires 24 real additions and no bit-shifting. The only complex matrix in the factorization, $\mathbf{W}_{7}$, requires 60 real additions and no bit-shifting operations. In total, the transform $\hat{\mathbf{F}}_{32}$ requires 348 real additions and no bit-shifting. For comparison, the Cooley-Tukey radix-2 algorithm requires 88 real multiplications and 408 real additions $[125,136]$.

\subsubsection{Error Analysis}

The filterbank frequency responses for four of the bins of the 32-point DFT, 32point ADFT, and the corresponding error plots are shown in Fig. 7.1. The four bins shown are the ones corresponding the the rows of the 32-point ADFT that performs the worst in terms of frequency response; thus they can be undestood as worst-case scenarios. The 32-point ADFT is "close enough" to the exact DFT to be useful in many practical applications, especially for wireless communications and SDR, where its error level of about $-10 \mathrm{~dB}$ is within the margin of error of the rest of the system (which includes both electronics and electromagnetics).

\subsection{Approximations for the 1024-point DFT}

\subsubsection{Approximation Methodology}

High-precision VLSI implementation of FFT algorithms may result in unnecessarily large circuits, high critical path delays, and wasted power. All of those factors contribute to higher-cost circuits, reduced frequency of operation, and higher operation costs. This is because digital multipliers demand a large amount of circuit resources 


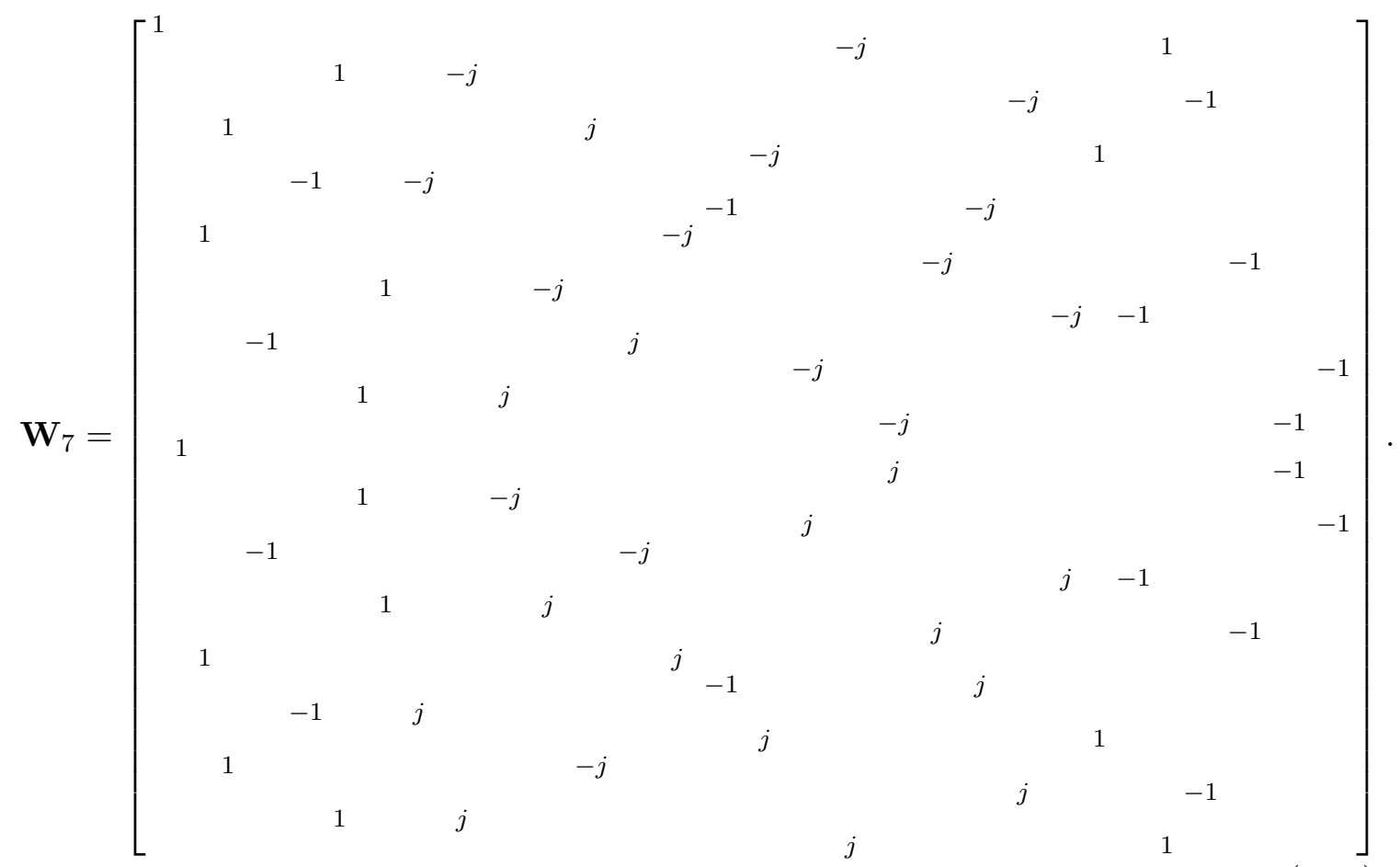

when compared to simple adders. This makes the reduction of the number of multipliers in a given system crucial when chip area and power must be conserved and high-speed operation is desirable.

We propose three ADFT algorithms which have small deviations of their filterbank responses when compared to the DFT. We assume that the applications at hand will be tolerant of the given deviations of frequency response, and that such deviations will be a small price to pay in exchange for the significantly smaller circuit realizations and power consumption over traditional fixed-point FFTs. It should be noted that the implementation of such approximate methods is not constrained by the minimum theoretical bounds of multiplicative complexity [87], that apply to the exact DFT. Indeed the proposed algorithms are not in fact calculating the DFT, but furnishing approximations that are nevertheless deemed reasonable for most 


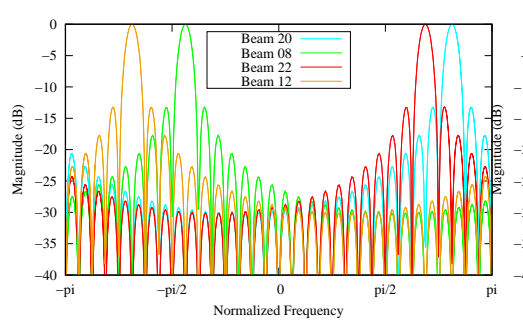

(a) 32-point DFT

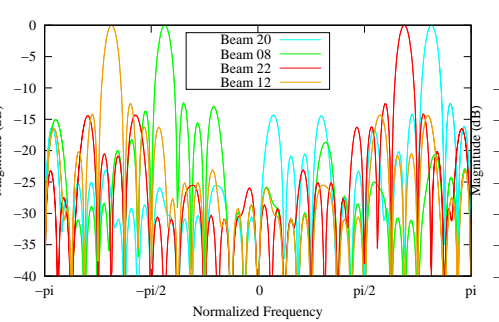

(b) 32-point ADFT

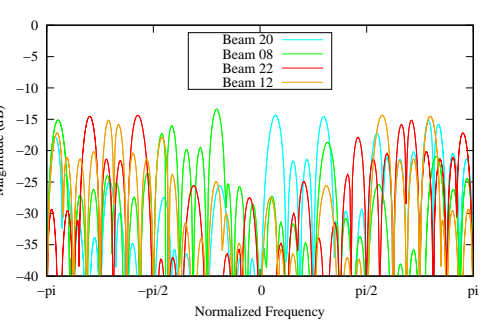

(c) Error

Figure 7.1: Filter-bank responses for (a) the exact 32-point DFT, (b) the 32-point ADFT and (c) absolute error of the ADFT response for the rows with the poorest performance.

high-speed digital-RF applications.

Based on (7.7), we propose the replacement of the exact 32-point DFT $\mathbf{F}_{32}$ by the 32-point ADFT proposed in [132].

Therefore, a suite of approximations for the DFT computation emerges.

We propose three different algorithms:

- Algorithm 1: ADFT-ADFT. Substitute both row- and column-wise 32point DFT $\mathbf{F}_{32}$ with the multiplierless 32-point ADFT $\hat{\mathbf{F}}_{32}$;

- Algorithm 2: Hybrid ADFT-DFT. Replace only the row-wise 32-point FFTs with the multiplierless 32-point ADFT in Section 7.2 leaving columnwise DFTs exact, and;

- Algorithm 3: Hybrid DFT-ADFT. Replace only the column-wise 32-point FFTs with the multiplierless 32-point ADFT in Section 7.2 leaving row-wise DFTs exact.

Let $\hat{\mathbf{X}}_{i}$ for $i=1,2,3$ denote approximations for $\mathbf{X}$ given by Algorithm 1, Algorithm 2, and Algorithm 3, respectively. Thus we have mathematically:

$$
\hat{\mathbf{X}}_{1}=\operatorname{vec}\left(\left[\boldsymbol{\Omega}_{32} \circ\left(\hat{\mathbf{F}}_{32} \cdot(\operatorname{invvec}(\mathbf{x}))^{\top}\right)\right] \cdot \hat{\mathbf{F}}_{32}^{\top}\right),
$$




$$
\begin{aligned}
& \hat{\mathbf{X}}_{2}=\operatorname{vec}\left(\left[\boldsymbol{\Omega}_{32} \circ\left(\hat{\mathbf{F}}_{32} \cdot(\operatorname{invvec}(\mathbf{x}))^{\top}\right)\right] \cdot \mathbf{F}_{32}^{\top}\right), \\
& \hat{\mathbf{X}}_{3}=\operatorname{vec}\left(\left[\boldsymbol{\Omega}_{32} \circ\left(\mathbf{F}_{32} \cdot(\operatorname{invvec}(\mathbf{x}))^{\top}\right)\right] \cdot \hat{\mathbf{F}}_{32}^{\top}\right) .
\end{aligned}
$$

The above combinations of ADFT and DFT yield low-complexity approximations

for the 1024-point DFT, which — due to its relatively large blocklength — is a computationally intractable task via usual direct numerical search methods. Algorithms 1, 2, and 3 have considerably different computational complexities and performance trade-offs as discussed in the next section.

\subsubsection{Arithmetic Complexity}

\section{Twiddle-factor Matrix}

In the three proposed algorithms, only the DFT computation $\mathbf{F}_{32}$ is subject to an approximation; the twiddle-factor matrix $\Omega_{32}$ is left unaltered in its exact form (cf. (7.7)). Therefore, a minimum number of multiplications remains due to $\Omega_{32}$. Considering only the nontrivial multiplications, the twiddle-factor matrix requires 961 complex multiplications, which translate into 2883 real multiplications and 2883 real additions.

\section{Algorithm 1}

Here the only source of multiplicative complexity are the twiddle factors in between the row- and column-wise 32-point ADFT blocks. Since the 32-point ADFT requires 348 additions and it is called 64 times, it contributes $64 \times 348=22272$ real additions to the overall arithmetic complexity of Algorithm 1. The resulting arithmetic costs are: 2883 real multiplications and $2883+22272=25155$ additions. 
Table 7.1: Real arithmetic complexity for the exact 1024-point DFT and for the proposed approximations

\begin{tabular}{lcccc}
\hline Algorithm & Real Mult. & Real Add. & \multicolumn{2}{c}{ \% Red. Comp.to Split Radix } \\
\cline { 1 - 2 } Split-Radix FFT [136] & 7,172 & 27,652 & Real Mult. & Real Add. \\
\hline Cooley-Tukey Radix-2 FFT [125] & 10,248 & 30,728 & -42.89 & -11.12 \\
\hline Winograd FFT [139] & 10,248 & 30,728 & -42.89 & -11.12 \\
\hline Proposed Algorithm 1 & 2,883 & 25,155 & 59.80 & 9.03 \\
\hline Proposed Algorithm 2 & 5,699 & 27,075 & 20.54 & 2.09 \\
\hline Proposed Algorithm 3 & 5,699 & 27,075 & 20.54 & 2.09 \\
\hline
\end{tabular}

\section{Algorithm 2}

Here multiplicative costs stem from the twiddle factors and the column-wise 32point exact DFT. The column-wise exact DFT is computed using the Cooley-Tukey radix-2 FFT $[125,136]$ (see Section 7.2.2). Since this algorithm requires 32 calls to the exact 32-point DFT and 32 calls to the 32-point ADFT, we have a total of $(32 \times 88)+2883=5699$ real multiplications and $(32 \times 408)+(32 \times 348)+2883=27075$ real additions.

\section{Algorithm 3}

Here the operation count follows the same rationale as for Algorithm 2, with the difference that the roles of the row and column-wise transforms are swapped. Therefore, Algorithms 2 and 3 have the same arithmetic costs. The arithmetic complexity of the proposed methods is summarized in Table 7.1.

\subsubsection{Performance of the Proposed Approximations}

The rows of a linear transform matrix can be understood as a finite impulse response (FIR) filter bank [124]. Thus we can assess how close the filter bank implied by the 


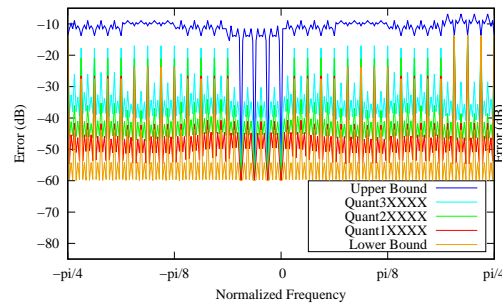

(a) Algorithm 1

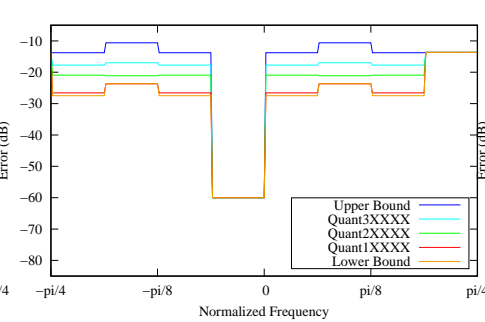

(b) Algorithm 2

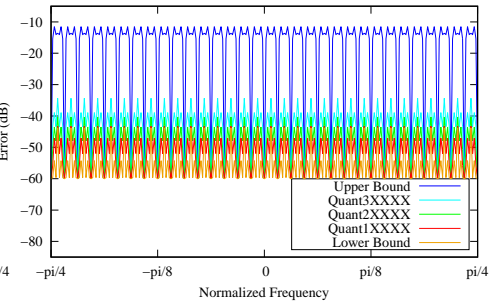

(c) Algorithm 3

Figure 7.2: Log-magnitude error of the frequency response of the rows of the proposed approximations where the least error is bounded to $-60 \mathrm{~dB}$

proposed approximations are relative to the exact 1024-point DFT. Considering the frequency response error expressed in log-magnitude, the Fig. 7.2 shows (i) the upper and lower envelopes and (ii) the first, second, and third quartiles of the error resulting from the proposed approximate filter banks $[145,146]$. For ease of visual inspection, we show only the normalized frequencies on the interval $[-\pi / 4, \pi / 4]$. The error of the frequency response for the remaining parts of the interval $[-\pi, \pi]$ are just a repetition of the plots in Fig. 7.2. Note that the three approximations resulting from Algorithm 1, Algorithm 2, and Algorithm 3 have distinct frequency response. Fig. 7.2 indicates that the Algorithm 1 is the one presenting the largest deviation from the exact DFT. This is expected given that the transform resulting from Algorithm 1 is obtained through the substitution of both the row- and column-wise DFT block by the discussed approximate 32-point DFT. This qualitative analysis is confirmed once we calculate the errors in the frequency responses of the rows of the three proposed approximations. Table 7.2 displays the minimum (nonzero), mean, and maximum for the squared magnitude of these errors. Notice that the transform resulting from Algorithm 1 has the highest deviations from the expected frequency response for its rows. In Table 7.2, we also show the worst-case side lobe in $\mathrm{dB}$ for each of the transforms. All considered transforms possess a low worst-case side lobe, on the order of $-12 \mathrm{~dB}$. 
Table 7.2: Statistics of errors in the rows of the proposed approximations: frequency response magnitude and worst-case side lobe level.

\begin{tabular}{ccccc}
\hline Transform & Min $(\mathrm{dB})$ & Mean $(\mathrm{dB})$ & Max $(\mathrm{dB})$ & $\begin{array}{c}\text { Worst Side } \\
\text { Lobe }(\mathrm{dB})\end{array}$ \\
\hline Algorithm 1 & -10.67 & -5.49 & -4.43 & -12.85 \\
\hline Algorithm 2 & -10.67 & -9.86 & -9.01 & -12.85 \\
\hline Algorithm 3 & -10.67 & -9.86 & -9.01 & -12.90 \\
\hline
\end{tabular}

\subsection{Digital VLSI Realization}

Next, we explore the digital VLSI realization of the proposed systems using a timemultiplexed approach. To distinguish the mathematical tool from its physical realization, hereafter we refer to the circuit implementation of the selected 32-point DFT and ADFT, respectively, as DFT32 and ADFT32 cores. Also, the hardware for the 1024-point exact DFT and each of the 1024-point approximations resulting from Algorithm 1, Algorithm 2, and Algorithm 3 are referred to as the DFT1024, ADFT1024_1, ADFT1024_2, and ADFT1024_3 cores, respectively.

Fig. 7.3 shows the overall architecture of the DFT1024 with the DFT32 cores. We focus on the design of the ADFT1024_1 core. Because this design can be easily extended to the other cores, the description of the ADFT1024_2 (Algorithm 2) and ADFT1024_3 (Algorithm 3) cores is omitted for brevity.

The core ADFT1024_1 processes an input signal block of 1024 time-domain samples in 32 clock cycles. Each signal block consists of 32 rows of adjacent time-domain samples in 32 columns. The first ADFT32 block sequentially computes the 32-point ADFT of each row, which are given by: $x[k], x[32+k], x[2 \times 32+k], \ldots, x[31 \times 32+k]$, for $k=0,1, \ldots, 31$. Intermediate frequency-domain values are passed to the transpose buffer, which realizes the matrix transposition operation in digital VLSI hardware, while operating in-step with the system clock. One complete matrix trans- 


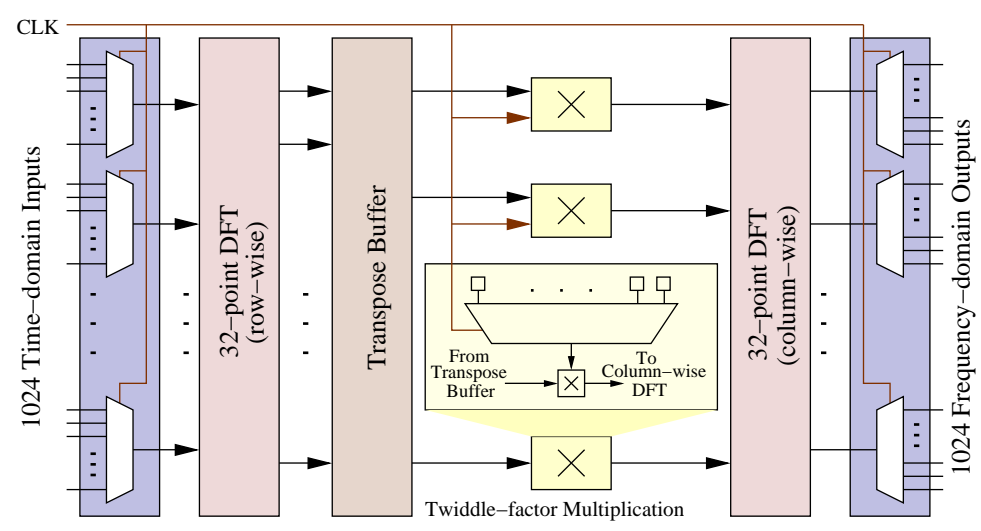

Figure 7.3: Signal flow graph showing the VLSI architecture to be modified for the proposed architecture based on the selected approximation. Algorithm 1: Replacement of both 32-point DFTs with 32-point ADFT blocks. Algorithm 2: Replacement of only row-wise 32-point DFT with 32-point ADFT blocks leaving column-wise DFT exact. Algorithm 3: Replacement of column-wise 32-point FFT with 32-point ADFT blocks leaving row-wise DFT exact.

pose operation is achieved every 32 clock cycles. The transpose buffer feeds the second time-multiplexed ADFT32 after suitable twiddle factors have been applied, which in turn, furnishes the desired 1024-point ADFT values. In order to minimize the chances of overflow, the second time-multiplexed ADFT32 block in Fig. 7.3 uses a larger wordlength by one bit than the first time-multiplexed ADFT32 block. This accommodates for the arithmetic operations that are carried on the first timemultiplexed ADFT32 and the twiddle factors.

\subsubsection{Transpose Buffer and Twiddle Factors}

The transpose buffer shown in Fig. 7.4 consists of a mesh of 1024 delays and 32 parallel multiplexers, each of them possessing 32 inputs. It generates the transpose of the first set of frequency bins. The transposition allows the column-wise DFT computation. 


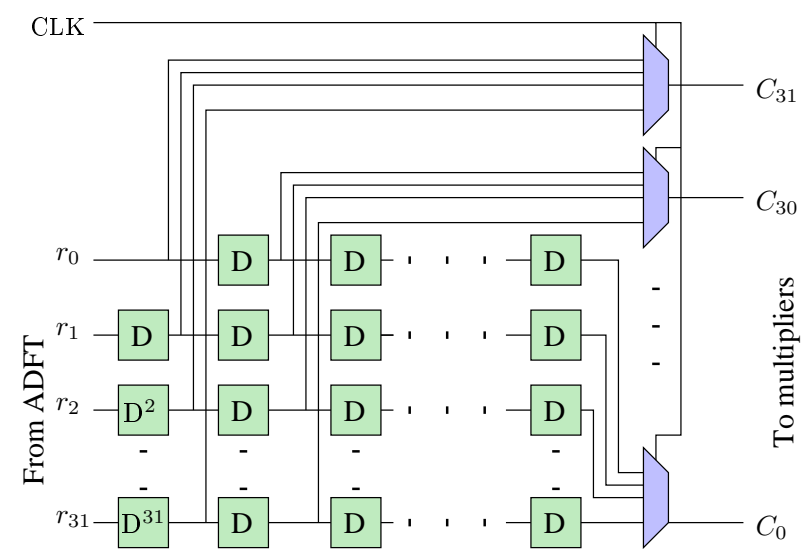

Figure 7.4: Schematic diagram of the transpose buffer.

Twiddle-factor multiplication consists of 961 complex multipliers, which are the only multiplications present in ADFT1024_1, which results from Algorithm 1. Each of the column bins (after the transpose buffer) undergoes a multiplication by $\omega_{1024}^{m \cdot n}$, where $0 \leq m \leq 31$ and $0 \leq n \leq 31$. Therefore, the precision of the twiddle-factor multipliers plays a critical role in the final area $A$, area-time $A T$, and area-timesquared $\left(A T^{2}\right)$ metrics. In this chapter, we have set the twiddle-factor precision level to be equal to the system word size of the inputs to the ADFT1024_1 core. This choice is a design parameter and the choice of lower precision levels in the twiddle factors would result in improvements in the VLSI metrics for all three proposed algorithms. In a sense, hardware designed with such conservative parameters can be thought of as worst-case benchmark, with more coarsely quantified twiddle factors leading to even better improvements in area, area-time, and area-time-squared metrics.

\subsubsection{Circuit Complexity}

ADFT1024_1 core

Each ADFT32 requires 130 adders/subtractors and no multipliers. The twiddle-factor matrix $\Omega_{32}$ in (7.14) demands 2883 real multipliers. As shown in Fig. 7.3, the pro- 
Table 7.3: Circuit complexity for the proposed architectures and the 1024-point DFT

\begin{tabular}{ccc}
\hline Design & Multipliers & Adders \\
\hline 1024-point DFT & 3,039 & 3,679 \\
\hline Algorithm 1 & 2,883 & 3,143 \\
\hline Algorithm 2 & 2,961 & 3,621 \\
\hline Algorithm 3 & 2,961 & 3,621 \\
\hline
\end{tabular}

posed architecture for Algorithm 1 uses only two ADFT32 cores. Thus, ADFT1024_1 has an overall circuit complexity of 260 adders/subtractors and 2883 multipliers.

\section{ADFT1024_2 core}

Regarding ADFT1024_2, only the row-wise DFT block is substituted by the selected ADFT block. The total number of multipliers is dictated by the column-wise DFT32 block and the twiddle factors. The twiddle factor matrix requires 2883 real multipliers. The DFT32 requires a total 78 real multipliers. The number of adders is influenced by all the blocks. The DFT32 contributes 398 adders. The twiddle factor matrix requires 2883 adders. The ADFT32 demands 340 adders. Thus, we have an overall circuit complexity of 3621 adders and 2961 multipliers ADFT1024_2.

\section{ADFT1024_3 core}

The complexity for the hardware implementation for the Algorithm 3 follows the same numbers of ADFT1024_2. The only change is in the placement of the elements in the architectural level.

The circuit complexities for the proposed designs as well as DFT1024 are presented in Table 7.3. 
Table 7.4: Key quantitative measurements of performance in digital $65 \mathrm{~nm}$ CMOS VLSI for each algorithm

\begin{tabular}{l|c|c|c|c|c|c|c}
\hline \multirow{2}{*}{$\begin{array}{c}\text { Performance } \\
\text { Metric }\end{array}$} & \multirow{2}{*}{ DFT1024 } & \multicolumn{2}{c|}{ ADFT1024_1 } & \multicolumn{2}{c|}{ ADFT1024_2 } & \multicolumn{2}{c}{ ADFT1024_3 } \\
\cline { 3 - 8 } & Value & Change & Value & Change & Value & Change \\
\hline Area, $A\left(\mathrm{~mm}^{2}\right)$ & 1.35 & 0.23 & $82.96 \% \downarrow$ & 0.58 & $57.04 \% \downarrow$ & 0.26 & $80.74 \% \downarrow$ \\
\hline $\begin{array}{l}\text { Critical Path Delay, } T \\
(\mathrm{~ns})\end{array}$ & 2 & 1.92 & $4 \% \downarrow$ & 1.86 & $7 \% \downarrow$ & 2 & - \\
\hline $\begin{array}{l}\text { Frequency, } F_{\max } \\
(\mathrm{GHz})\end{array}$ & 0.5 & 0.52 & $4.12 \% \uparrow$ & 0.54 & $7.53 \% \uparrow$ & 0.5 & - \\
\hline $\begin{array}{l}A T \\
\left(\mathrm{~mm}^{2} \mathrm{~ns}\right)\end{array}$ & 2.7 & 0.44 & $83.64 \% \downarrow$ & 1.08 & $60.04 \% \downarrow$ & 0.52 & $80.74 \% \downarrow$ \\
\hline $\begin{array}{l}A T^{2} \\
\left(\mathrm{~mm}^{2} \mathrm{~ns}^{2}\right)\end{array}$ & 5.4 & 0.85 & $84.30 \% \downarrow$ & 2.01 & $62.84 \% \downarrow$ & 1.04 & $80.74 \% \downarrow$ \\
\hline $\begin{array}{l}\text { Dynamic Power, } D_{p} \\
(\mathrm{~mW} / \mathrm{GHz})\end{array}$ & 150.62 & 48.19 & $68.00 \% \downarrow$ & 71.75 & $52.36 \% \downarrow$ & 121.84 & $19.10 \% \downarrow$ \\
\hline
\end{tabular}

\subsubsection{ASIC Synthesis and Place-Route Results: 65nm CMOS}

The proposed architectures were implemented on MATLAB Simulink using Xilinx libraries and then mapped to 65-nm complementary metal-oxide semiconductor (CMOS) technology cells (synthesis only). Each of the designs consists of three main hardware components - first 32-point transform block, transpose buffer with twiddle-factor multiplication block, and second 32-point transform block. The complexity of each 32-point transform block core depends on its corresponding input word length. In Table 7.4, we enlist the hardware implementation metrics for ADFT1024_1, ADFT1024_2, and ADFT1024_3. Metrics for the DFT1024 core were included as reference values.

\subsubsection{Analysis of the Results}

The results in Table 7.4 shows that all three 1024-point ADFT cores demands considerably less hardware resources than the 1024-point exact DFT core. On the other hand, the implementation of the transpose buffer with twiddle factor multiplication adds a fixed hardware complexity to the system for both the DFT and 


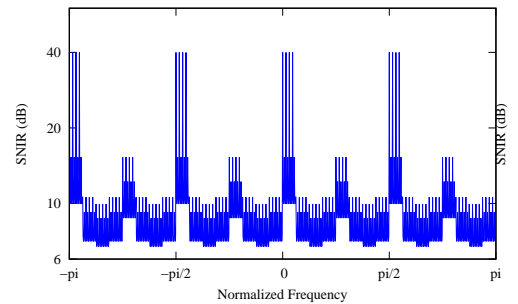

(a) Algorithm 1

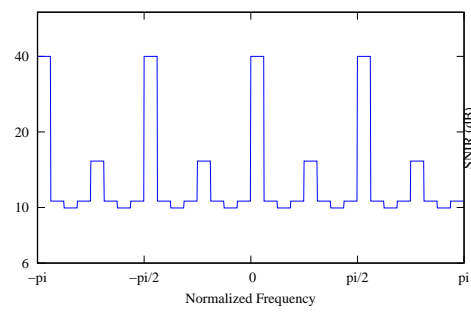

(b) Algorithm 2

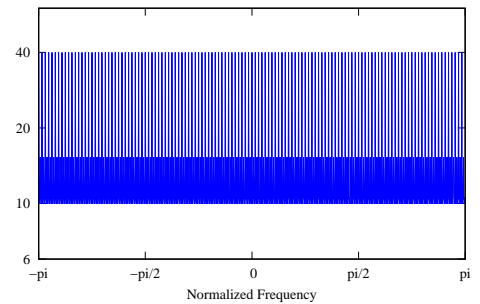

(c) Algorithm 3

Figure 7.5: Plots for $\gamma_{k}$, which is used to measure the deviations from orthogonality of (a) Algorithm 1, (b) Algorithm 2, and (c) Algorithm 3 from the ideal case of the 1024-point DFT matrix, with a cap of $40 \mathrm{~dB}$ for visualization purposes. The plots show the output SIR due to spectral leakage among DFT bins due to various levels of approximation.

the approximate architectures. As a result, the transpose buffer causes the highest area consumption and a relatively high power consumption in comparison to that of 32-point ADFT cores. Thus, it becomes the dominant factor in hardware complexity for the designs of the three 1024-point approximate transforms, as shown in Table 7.4.

The core ADFT1024_1 gives the best hardware utilization, whereas ADFT1024_2 gives the worst as can be seen in Table 7.4. Algorithm 3 gives the best error performance, i.e., provides the most accurate approximation. Moreover, the hardware resource consumption of its physical realization ADFT1024_3 is also close to that of ADFT1024_1. The error performance of Algorithm 2 does not differ much from that of Algorithm 1, which also provides a hardware realization ADFT1024_1 with the lowest resource consumption. Therefore, we recommend either Algorithm 1 or Algorithm 3 (i.e., its hardware realizations ADFT1024_1 and ADFT1024_3) as the best designs. 


\subsection{Applications in Wireless Modulation}

In this section, we examine two real-world applications for the proposed algorithms and their implemented architectures.

\subsubsection{OFDM}

\section{OFDM Background}

The $N$-point DFT realizes a filterbank with $N$ fully-orthogonal frequency domain

responses. For the $k$ th bin (frequency response), the maxima occurs at discrete frequency points $2 k \pi / N$ with zeros at $2 n \pi / N$ where $n \neq k, k=0,1, \ldots, N-$ $1[147,148]$. The fact that these responses are orthogonal to each other allows high spectral efficiency in wireless communications based on OFDM [149]. In OFDM, each spectral bin is individually modulated using a high-order modulation, typically 64-point quadrature amplitude modulation (64QAM) consisting of a constellation fitting a grid of size $8 \times 8$ in the real-imaginary (I-Q) planes of the $N$ frequency channels [150]. Orthogonality allows independent modulation of the channels (a form of multiple access) without the spectra of modulated channels interfering with each other. Thus, this property is important for high spectral efficiency via highorder modulation and low bit-error rates when the signal-to-noise ratio (SNR) is high (e.g., > $30 \mathrm{~dB})[151]$.

\section{Approximately Orthogonal Frequency Division Multiplexing (A-OFDM)}

Although the proposed approximations are not orthogonal, they may be "orthogonal enough". Thus, we proceed to quantify their deviations from orthogonality. Let $H_{k}(\omega ; \mathbf{T})$ for $\omega \in[-\pi, \pi]$ be the frequency response at the $k$ th discrete frequency point (bin) for the transform $\mathbf{T}$. It is known that the DFT evaluates $H_{k}(\omega ; \mathbf{T})$ over 
the frequencies $\omega_{n}=2 \pi n / N$ for $n=0,1, \ldots, N-1$ [124]. It is also known that the $N$-point DFT has the following property:

$$
\left.H_{k}\left(\omega ; \mathbf{F}_{N}\right)\right|_{\omega_{n}}=\left\{\begin{array}{l}
1, \text { if } n=k \\
0, \text { if } n \neq k
\end{array}\right.
$$

where $k=0,1, \ldots, N-1[124,125]$. In general, DFT approximations do not follow (7.17). DFT approximation $\hat{\mathbf{F}}_{N}$ usually follows a relaxed condition, given by

$$
\left.H_{k}\left(\omega ; \hat{\mathbf{F}}_{N}\right)\right|_{\omega_{n}}=\left\{\begin{array}{l}
\alpha, \text { if } n=k \\
\epsilon_{k}, \text { if } n \neq k
\end{array}\right.
$$

where $k=0,1, \ldots, N-1$, and $\alpha \approx 1$ and $\epsilon_{k} \approx 0$.

Let $\mathbf{T}_{N}$ be an $N$-point transform. A possible way to evaluate how orthogonal a transform $\mathbf{T}_{N}$ is by computing

$$
M_{k}\left(\mathbf{T}_{N}\right)=\frac{\|\alpha\|^{2}}{\sum_{\substack{n=0 \\ n \neq k}}^{N-1}\left\|\epsilon_{k}\right\|^{2}}
$$

where $k=0,1, \ldots, N-1$. The expression in (7.19) measures the amount of energy of the bin $k$ that is leaked-or spread-to other bins other than $k$. It is a measure of the interference of all the beams on the $k$ th beam. Its value is related to the signal-to-interference ratio (SIR) of the output signal after being transformed by $\mathbf{T}$. Because of (7.17), we have that for the DFT, $M_{k}\left(\mathbf{F}_{N}\right) \rightarrow \infty$ for $k=0,1, \ldots, N-1$. For an arbitrary DFT approximation, this does not hold true.

Fig. 7.5 shows the values of $\gamma_{k}=10 \log _{10}\left(M_{k}\left(\hat{\mathbf{F}}_{N}\right)\right)$ for the proposed three algorithms. Notice that, for visualization purposes, the values of $\gamma_{k}$ in Fig. 7.5 are capped to a maximum of $40 \mathrm{~dB}$.

The value of $\gamma_{k}$ works as an upper limit to the maximum reachable SIR that one can obtain after computing the $k$ th bin. Under orthogonality, there is no leakage 


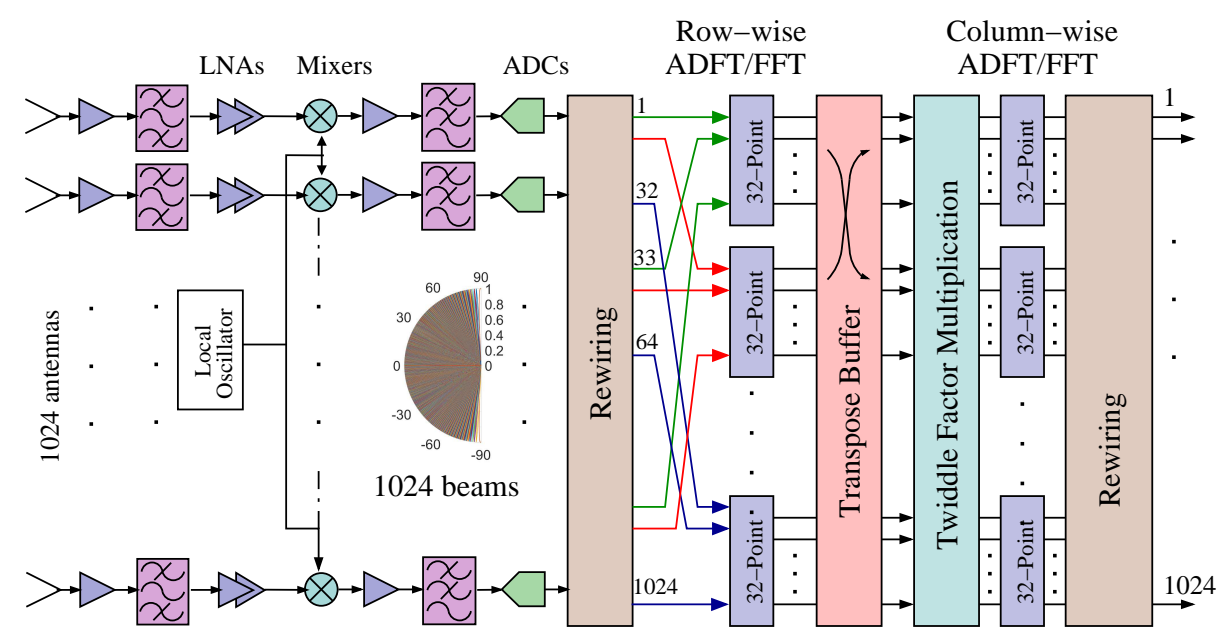

Figure 7.6: Beamforming architecture of a 1024 ULA receiver using the proposed architecture. The rewiring block performs multiplexing as shown in Fig. 7.3.

from and to the neighboring bins, and therefore $\gamma_{k} \rightarrow \infty$. This makes the bin output take the input signal-to-noise plus interference ratio (SNIR) value, indicating both perfectly orthogonal response and no loss of performance. Higher dB for $\gamma_{k}$ values quantify the computation accuracy of the $k$ th bin of A-OFDM schemes, and serves as a guide for the choice of A-OFDM design parameters. In general, the lower the SNR of the input signal, the lower the possible depth of modulation (i.e., lower-order QAM on the $k$ th bin).

For the purpose of illustration, we assume $\mathrm{SNR}_{\text {input }}=40 \mathrm{~dB}$, which is reasonable for a high-performance wireless system capable of 1024-point OFDM with 1024QAM per subcarrier using ideal DFTs [152-155]. The values of $\gamma_{k}$ for the three proposed ADFT algorithms in Fig. 7.5 show that the smallest SIR is of the order of $7 \mathrm{~dB}$, which is still acceptable for many applications [151] where the input SNR of the system is relatively low. In particular, this regime is relevant for SNR-limited wireless systems including emerging $5 \mathrm{G}$ and $6 \mathrm{G}$ wireless communications, which use mm-wave and sub- $\mathrm{THz}$ channels that are expected to have low SNR when direct line-of-sight may not be available [5]. In fact, for lower order modulations, such as 
64QAM, and quadrature phase shift keying (QPSK), the required minimum SNR per subcarrier is approximately $27 \mathrm{~dB}$, and $3 \mathrm{~dB}$, respectively [156].

Therefore, the use of DFT approximations would allow a designer to assign high-capacity (i.e., high spectral efficiency) modulations to the particular bins that maintain the high input SNR (i.e., approximately $40 \mathrm{~dB}$ input SNR), while assigning lower order modulations to bins with degraded SNR values. This results in a trade-off in maximum system capacity depending on whether one uses the DFT, or one of the three proposed ADFTs. Thus, use of the ADFT entails an engineering compromise that trades OFDM system capacity for smaller chip area, battery life (lower dynamic power), and speed (due to lower critical path delay).

\subsubsection{Multi-Beam Beamforming}

Electronically-steerable transmit and receive aperture arrays for RF spectrum sensing, communications, and radar use the FFT for multi-beam beamforming. For example, simultaneous receiver beams are imperative for high-capacity MIMO wireless communication systems.

Multiple independent RF beams can be generated by applying an $N$-point spatial FFT at each time sample across a uniform linear array (ULA) of antennas $[157,158]$. For an $N$-element ULA with Nyquist (i.e., $\lambda / 2$ ) spacing, the $N$ beams are uniformly spaced in the spatial frequency domain with an interval of $2 \pi / N$. The proposed architecture can be used to replace the FFT for this purpose, thus generating $N=$ 1024 beams from a 1024-element ULA as shown in Fig. 7.6. In the proposed system, each ADFT bin corresponds to a unique direction in space. Ideally these bins should be identical to the spatial DFT bins, but their magnitude could deviate because of the approximation. The four worst bins for each of the three algorithms are shown 
in Fig. 7.7. The resulting errors are small enough to be acceptable in low-SNR scenarios.

Realization of 1024-element ULAs for generating narrow beams in currentlylicensed frequency bands (upto the $\mathrm{V}$ band) may be challenging due to the large sizes of the resulting apertures. However, due to ongoing research in the sub- $\mathrm{THz}$ range [159-167], the $\mathrm{W}$ and $\mathrm{G}$ bands will soon be commercially available. At a carrier frequency of $300 \mathrm{GHz}, \lambda / 2=0.5 \mathrm{~mm}$ and thus the size of a Nyquist-spaced 1024-element ULA would decrease to a reasonable value of $51.2 \mathrm{~cm}$.

\subsection{Conclusion}

FFTs are used for reducing the computational costs of evaluating the DFT. Generally, they decrease complexity from $\mathcal{O}\left(N^{2}\right)$ down to $\mathcal{O}(N \log N)$. In this chapter, we have showed that further savings can be accomplished by means of approximate methods. The resulting 1024-point DFT approximations present a trade-off between performance and hardware complexity without significant loss in terms of worst-side lobe and SNR.

Our work shows that larger block-length DFT approximations can be obtained from the smaller-size approximations derived using previously-described numerical optimization methods. Our methodology can be directly applied to any DFT for which the block length is a perfect square. Since the current DFT approximations in the literature are restricted to the sizes $\{8,16,32\}[132,146,168-170]$, approximate algorithms can be derived for $N \in\{64,256,1024\}$. In this work, we focused on the 1024-point case. Assuming that a multiplierless DFT approximation of size $\sqrt{N}$ can always be found, our derivations suggests that we can obtain an $N$-point DFT approximation that requires only $N-2 \sqrt{N}-1$ multiplications; effectively making the 


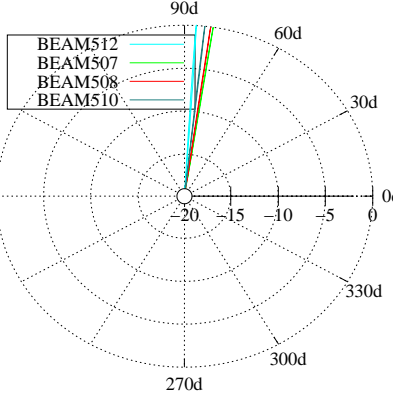

(a) DFT

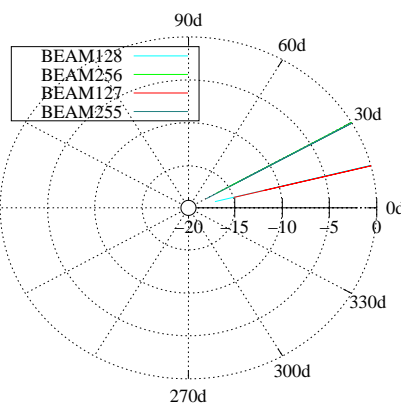

(d) DFT

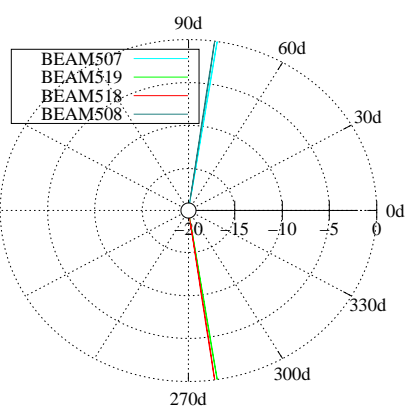

(g) DFT

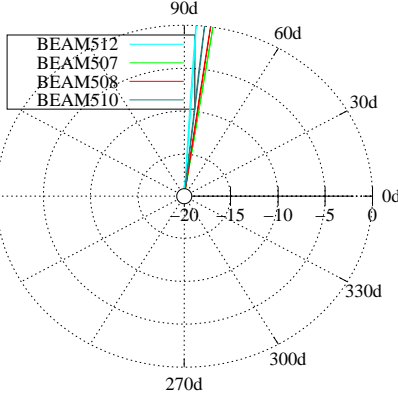

(b) Algorithm 1

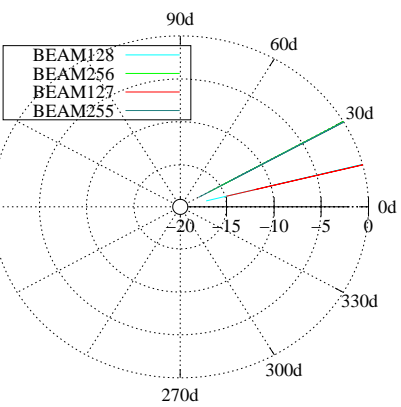

(e) Algorithm 2

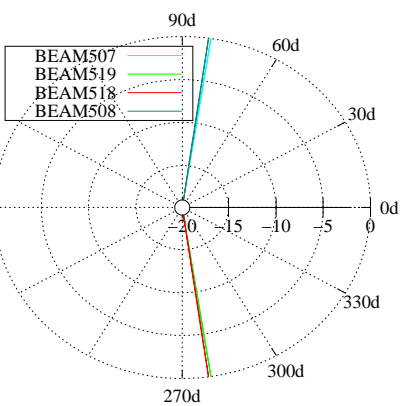

(h) Algorithm 3

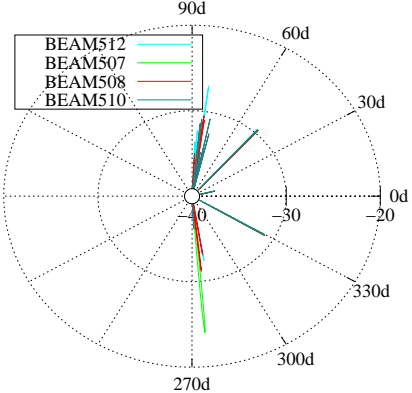

(c) Error for Algorithm 1

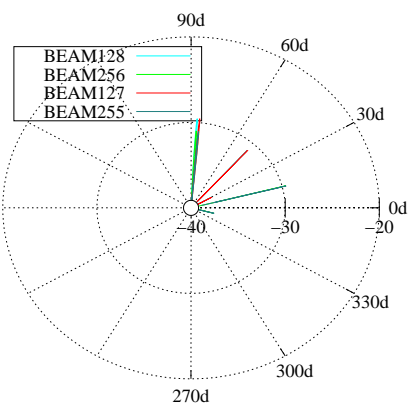

(f) Error for Algorithm 2

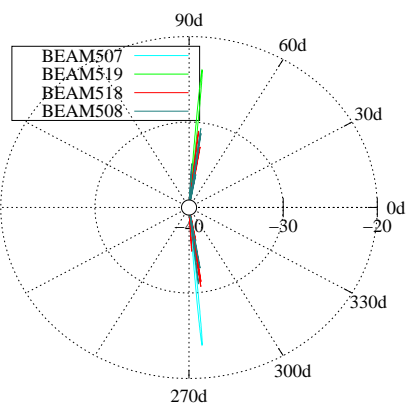

(i) Error for Algorithm 3

Figure 7.7: The four worst bins for multi-beam beamforming: (a) exact DFT response, (b) ADFT response, and (c) error for algorithm 1; (d) exact DFT response, (e) ADFT response, and (f) error for algorithm 2; (g) exact DFT response, (h) ADFT response, and (i) error for algorithm 3. 
complexity of the resulting $N$-point approximation $\mathcal{O}(N)$. The proposed algorithms were synthesized to digital VLSI using a 65-nm CMOS library. Synthesis results confirm the expected improvements in layout area and power consumption metrics compared to a conventional 1024-point DFT implementation.

The choice of algorithm depends on the application and its tolerance for computational error in the DFT block. Highly error tolerant applications can greatly benefit from Algorithm 1 which has the lowest complexity. Algorithm 3 maybe selected when Algorithm 1 does not furnish sufficient performance. 


\section{CHAPTER 8 CONCLUSIONS AND FUTURE WORK}

Phased antenna array systems are being used in mmWave applications to increase the total physical aperture size and to obtain more degrees of freedom such as spatial diversity and multi-user compatibility. Sub-THz massive MIMO and mmWave MIMO systems may incorporate hundreds or perhaps thousands of antenna elements to achieve highly directional beams. Antenna arrays can form multiple, highly directional beams using beamforming approaches where the directionality and the sharpness of the beams increase with the number of antennas. Analog beamforming used in phased array systems consumes less power but has a lower degree of freedom as compared to digital beamformers, which require a dedicated RF chain and an ADC for each element of the array. The mixed-signal component of a phased array system (i.e. $\mathrm{ADC} / \mathrm{DAC}$ ) creates a bottleneck as the $\mathrm{SWaP}$ and cost parameters have a high dependency on this component.

In traditional realizations, a fully digital $N$-element phased array system can achieve $N$ independent digital beams and require $N$ ADCs at IF or $2 N$ ADCs at baseband to sample I and Q components. Digital hardware in digital beamformers often use DFT cores to generate multiple beams by performing a spatial DFT. The complexity (which is determined by the number of multiplications in the DFT matrix) can be reduced by following FFT computational algorithms, yet the lowest complexity of an $N$-point DFT is bounded by $N$ for exact implementations. Therefore, mixed and digital domain hardware complexities are massive at higher $N$ values, leading to extensive $\mathrm{SWaP}$ and costs.

The earlier chapters discussed several mixed and digital domain algorithms that can be used to reduce the ADC complexity as well as the possibility of approximating the DFT matrix to reduce digital domain complexity. 
The well known $\Sigma-\Delta$ concept is widely used in ADCs to reduce the complexity by shaping the quantization noise out of the signal band. The possibility of extending the $\Sigma-\Delta$ architecture to the MD domain by extending the feedback topology to both space and time for discrete-space-and-continuous-time was discussed in Chapter. 3. The findings presented in this chapter show that it is possible to eliminate the requirement for spatial oversampling, because of the sparsity in the MD spectrum. This architecture also suggests employing a single $N$-port ADC to replace $N$ ADCs used in an $N$-element system such that the spatial correlation among received signals can be taken into account, and the ADCs can be replaced with 1-bit quantizers. This concept was analyzed theoretically and proposed for experimental verification.

The concept of $N$-port $\Sigma-\Delta$ ADCs was extended for microwave focal plane array (FPA) dish receivers in Chapter 4 to achieve ADC reduction. Realization of this architecture replace conventional ADCs with 1-bit quantizers for temporally wideband planar waves. Simulation results for focal plane ULAs have shown that this concept is theoretically suitable for experimental verification.

The AMNA discussed in Chapter 5 uses the MD spectral properties discussed in Chapter 2. The RoS of a planar wave impinging on a ULA or a URA at an angle of $\psi$ (as measured from the broadside of the array) takes the form of a line in the $2 \mathrm{D}$ or $3 \mathrm{D}$ spectrum, respectively. The range of the RoS is bounded by a dual fan region for the $2 \mathrm{D}$ spectrum and a cone in the 3D spectrum with a half angle of $\pi / 4$ for the full FOV of $-\frac{\pi}{2} \leq \psi \frac{\pi}{2}$. Traditional use of ADCs assumes omni-directional antenna patterns, even though these patterns are directional in practice. The novel approach proposed in Chapter 5 involves exploiting the MD signal properties to linearly transform the RoS such that the information captured by multiple antenna elements can be accommodated in the same MD spectrum with little to no interference and that can be sampled using a single ADC. Mathematical analysis of ULAs has shown that 
this approach can be used for single/dual polarized ULAs to achieve a $\frac{k-1}{k} \times 100 \%$ ADC complexity reduction, where $k$ is the number of sub-arrays. Simulation results have demonstrated that ADC complexity reductions of $50 \%$ for ULAs and $75 \%$ for URAs can be achieved without losing degrees of freedom. Experimental verification followed by CMOS realization of the analog component and FPGA realization of required spatio-temporal filters for this concept can be conducted in future research.

Multiplexing algorithms can also be used to combine multiple RF channels to reduce the ADC requirement. An FDM-based approach was presented in Chapter 6 to achieve an $M$-fold decrease in the number of ADCs required. Experimental verification was performed for a four-element, 28-GHz phased array system for a case where $M=4$. Once the combined signal is digitized, Xilinx RFSoC 1285 FPGA was used as the digital platform to implement polyphase filters that employ sharp FIR filters to separate the frequency bands. A spatial FFT was used to generate four beams having the highest degree of freedom, and analyzed using MATLAB. While the proposed system achieved a factor of 4 in terms of ADC reduction, the received spectrum was affected by the non-linearities of the system, and the SIR was about $15 \mathrm{~dB}$. In future work, appropriate steps may be identified to avoid intermodulation products and harmonics from aliasing into the signal spectrum, and a breakout board to be used with Xilinx RFSoC may be developed.

Digital beamforming platforms use a FFT core to evaluate the spatial DFT. The hardware complexity, which is often determined by the number of multiplications in the DFT matrix can generally be reduced from $\mathcal{O}\left(N^{2}\right)$ to $\mathcal{O}(N \log N)$ using the Cooley-Tukey algorithm. Some advanced approaches may be able to achieve even lower complexities; however, the lowest achievable complexity for an $N$-point DFT is bounded to be $\mathcal{O}(N)$. It should be noted that the implementation of the coefficients of these matrices in digital hardware always requires an approximation because of 
the finite level of precision. Therefore, the DFT can be approximated further to achieve a better reduction in hardware complexity at an allowable cost of precision. Three implementation algorithms for realizing a 1024-point ADFT using Radix-32 approximate DFTs were presented in Chapter 7. The corresponding digital designs were developed using a Xilinx System Generator for MATLAB Simulink and were synthesized to digital very-large-scale-integration (VLSI) using the TSMC 65-nm CMOS library. Hardware results have shown a reduction of approximately $80 \%$ in the area and $68 \%$ reduction in dynamic power in comparison to its Cooley-Tukey realization. 


\section{BIBLIOGRAPHY}

[1] T. S. Rappaport, J. N. Murdock, and F. Gutierrez, "State of the art in 60-GHz integrated circuits and systems for wireless communications," Proceedings of the IEEE, vol. 99, no. 8, pp. 1390-1436, Aug 2011.

[2] Z. Pi and F. Khan, "An introduction to millimeter-wave mobile broadband systems," IEEE Communications Magazine, vol. 49, no. 6, pp. 101-107, June 2011.

[3] S. Singh, H. S. Dhillon, and J. G. Andrews, "Offloading in heterogeneous networks: Modeling, analysis, and design insights," IEEE Transactions on Wireless Communications, vol. 12, no. 5, pp. 2484-2497, May 2013.

[4] T. S. Rappaport, Wireless Communications: Principles and Practice (2nd Edition). Prentice Hall, 2002. [Online]. Available: https://www.amazon. com/Wireless-Communications-Principles-Practice-2nd/dp/0130422320? SubscriptionId=AKIAIOBINVZYXZQZ2U3A\&tag $=$ chimbori05-20\& $\operatorname{linkCode}=\mathrm{xm} 2 \&$ camp $=2025 \&$ creative $=165953 \&$ creativeASIN $=0130422320$

[5] T. S. Rappaport, S. Sun, R. Mayzus, H. Zhao, Y. Azar, K. Wang, G. N. Wong, J. K. Schulz, M. Samimi, and F. Gutierrez, "Millimeter wave mobile communications for $5 \mathrm{G}$ cellular: It will work!" IEEE Access, vol. 1, pp. 335349, 2013.

[6] E. Brookner, "Advances and breakthroughs in radars and phased-arrays," in 2016 CIE International Conference on Radar (RADAR), Oct 2016.

[7] C. E. Shannon, "Communication in the presence of noise," Proceedings of the IRE, vol. 37, no. 1, pp. 10-21, Jan 1949.

[8] P. Busson, H. Luong, C. M. Hung, H. Krishnaswamy, T. Georgantas, and P. P. Mercier, "F4: Circuit and system techniques for mm-wave multi-antenna systems," in 2018 IEEE International Solid - State Circuits Conference (ISSCC), Feb 2018, pp. 511-513.

[9] "Millimeter wave 70/80/90 ghz service." [Online]. Available: https://www.fcc.gov/wireless/bureau-divisions/broadband-division/ microwave-services/millimeter-wave-708090-ghz-service 
[10] X. Wang, L. Kong, F. Kong, F. Qiu, M. Xia, S. Arnon, and G. Chen, "Millimeter wave communication: A comprehensive survey," IEEE Communications Surveys Tutorials, vol. 20, no. 3, pp. 1616-1653, thirdquarter 2018.

[11] S. E. Ershadi, A. Keshtkar, A. Bayat, A. H. Abdelrahman, and H. Xin, "Rotman lens design and optimization for $5 \mathrm{G}$ applications," International Journal of Microwave and Wireless Technologies, vol. 10, no. 9, p. 1048â "1057, 2018.

[12] J. Parikh and A. Basu, Millimeter Waves: Technological Component for Next-Generation Mobile Networks. Cham: Springer International Publishing, 2019, pp. 167-186. [Online]. Available: https://doi.org/10.1007/ 978-3-319-91002-4_7

[13] A. Al-Samman, T. Rahman, M. Hindia, A. Daho, and E. Hanafi, "Path loss model for outdoor parking environments at $28 \mathrm{GHz}$ and $38 \mathrm{GHz}$ for $5 \mathrm{G}$ wireless networks," Symmetry, vol. 10, no. 12, p. 672, Nov 2018. [Online]. Available: http://dx.doi.org/10.3390/sym10120672

[14] B. Satchidanandan, S. Yau, S. S. Ganji, P. R. Kumar, A. Aziz, A. Ekbal, and N. Kundargi, "A directional medium access control protocol for 5G millimeter-wave local area networks," in Communication Systems and Networks, S. Biswas, A. Mukherjee, M. C. Chan, S. Chakraborty, A. Kumar, G. Mandyam, and R. Shorey, Eds. Cham: Springer International Publishing, 2019, pp. 150-171.

[15] A. M. Al-Samman, T. Abd. Rahman, T. Al-Hadhrami, A. Daho, M. N. Hindia, M. H. Azmi, K. Dimyati, and M. Alazab, "Comparative study of indoor propagation model below and above $6 \mathrm{GHz}$ for $5 \mathrm{G}$ wireless networks," Electronics, vol. 8, no. 1, 2019. [Online]. Available: http://www.mdpi.com/2079-9292/8/1/44

[16] T. S. Rappaport, Y. Xing, O. Kanhere, S. Ju, A. Madanayake, S. Mandal, A. Alkhateeb, and G. C. Trichopoulos, "Wireless communications and applications above $100 \mathrm{GHz}$ : Opportunities and challenges for 6G and beyond," IEEE Access, pp. 1-1, 2019.

[17] H. T. Friis, "A note on a simple transmission formula," Proceedings of the IRE, vol. 34, no. 5, pp. 254-256, 1946.

[18] C. Narayan, Antennas And Propagation. Technical Publications, 2007. 
[19] P. Merias, "Study on new radio access technology physical layer aspects (specification \#: 38.802)," 3GPP, Tech. Rep., 2017. [Online]. Available: https://portal.3gpp.org/desktopmodules/Specifications/ SpecificationDetails.aspx?specificationId $=3066$

[20] F. Gutierrez, S. Agarwal, K. Parrish, and T. S. Rappaport, "On-chip integrated antenna structures in CMOS for $60 \mathrm{GHz}$ WPAN systems," IEEE Journal on Selected Areas in Communications, vol. 27, no. 8, pp. 1367-1378, October 2009.

[21] W. Hong, K. Baek, Y. Lee, Y. Kim, and S. Ko, "Study and prototyping of practically large-scale mmwave antenna systems for $5 \mathrm{G}$ cellular devices," IEEE Communications Magazine, vol. 52, no. 9, pp. 63-69, September 2014.

[22] W. Roh, J. Seol, J. Park, B. Lee, J. Lee, Y. Kim, J. Cho, K. Cheun, and F. Aryanfar, "Millimeter-wave beamforming as an enabling technology for $5 \mathrm{G}$ cellular communications: theoretical feasibility and prototype results," IEEE Communications Magazine, vol. 52, no. 2, pp. 106-113, February 2014.

[23] S. Han, C. I, Z. Xu, and C. Rowell, "Large-scale antenna systems with hybrid analog and digital beamforming for millimeter wave 5G," IEEE Communications Magazine, vol. 53, no. 1, pp. 186-194, January 2015.

[24] M. N. Kulkarni, S. Singh, and J. G. Andrews, "Coverage and rate trends in dense urban mmwave cellular networks," in 2014 IEEE Global Communications Conference, Dec 2014, pp. 3809-3814.

[25] S. Dutta, C. N. Barati, D. Ramirez, A. Dhananjay, J. F. Buckwalter, and S. Rangan, "A case for digital beamforming at mmwave," IEEE Transactions on Wireless Communications, vol. 19, no. 2, pp. 756-770, 2020.

[26] P. L. Tokarsky, A. A. Konovalenko, S. N. Yerin, and I. N. Bubnov, "An active antenna subarray for the low-frequency radio telescope GURT-Part II: Numerical analysis and experiment," IEEE Transactions on Antennas and Propagation, vol. 67, no. 12, pp. 7312-7319, 2019.

[27] N. Akram, A. Madanayake, S. Handagala, S. Mandal, and L. Belostotski, "Multiport ADCs for microwave focal plane array dish receivers," in 2018 IEEE International Symposium on Circuits and Systems (ISCAS), May 2018, pp. $1-5$. 
[28] A. Madanayake, N. Akram, S. Mandal, J. Liang, and L. Belostotski, "Improving adc figure-of-merit in wideband antenna array receivers using multidimensional space-time delta-sigma multiport circuits," in 2017 10th International Workshop on Multidimensional (nD) Systems (nDS), Sep. 2017, pp. 1-6.

[29] M. V. Komandla, G. Mishra, and S. K. Sharma, "Investigations on dual slant polarized cavity-backed massive MIMO antenna panel with beamforming," IEEE Transactions on Antennas and Propagation, vol. 65, no. 12, pp. 67946799, Dec 2017.

[30] [Online]. Available: https://www.eedesignit.com/ world-record-in-5g-wireless-spectrum-efficiency-set/

[31] L. Zhang and H. Krishnaswamy, "Arbitrary analog/RF spatial filtering for digital MIMO receiver arrays," IEEE Journal of Solid-State Circuits, vol. 52, no. 12, pp. 3392-3404, Dec 2017.

[32] — , "Recent advances in analog/RF interference mitigation for massive MIMO receivers," in 2017 IEEE 60th International Midwest Symposium on Circuits and Systems (MWSCAS), Aug 2017, pp. 21-24.

[33] M. V. Komandla, G. Mishra, and S. K. Sharma, "Dual slant polarized cavity backed massive MIMO panel array antenna with digital beamforming," in 2017 IEEE International Symposium on Antennas and Propagation USNC/URSI National Radio Science Meeting, July 2017, pp. 1429-1430.

[34] J. D. Díaz, J. L. Salazar, J. A. Ortiz, C. Fulton, N. Aboserwal, R. Kelley, and R. Palmer, "A dual-polarized cross-stacked patch antenna with wideangle and low cross-polarization for fully digital multifunction phased array radars," in 2016 IEEE International Symposium on Phased Array Systems and Technology (PAST), Oct 2016, pp. 1-4.

[35] O. Jo, J. J. Kim, J. Yoon, D. Choi, and W. Hong, "Exploitation of dualpolarization diversity for $5 \mathrm{G}$ millimeter-wave MIMO beamforming systems," IEEE Transactions on Antennas and Propagation, vol. 65, no. 12, pp. 66466655, Dec 2017.

[36] H. Kikuchi, T. Wu, E. Yoshikawa, T. Ushio, H. Goto, F. Mizutani, M. Wada, and V. Chandrasekar, "Performance of minimum mean-square error beam forming for polarimetric phased array weather radar," IEEE Transactions on Geoscience and Remote Sensing, vol. 55, no. 5, pp. 2757-2770, May 2017. 
[37] J. George and V. Chandrasekar, "Front-X phased array weather radar test bed," in 2016 IEEE International Symposium on Phased Array Systems and Technology (PAST), Oct 2016, pp. 1-7.

[38] J. A. Haimerl, B. Hudson, G. P. Fonder, and D. K. Lee, "Overview of the large digital arrays of the space fence radar," in 2016 IEEE International Symposium on Phased Array Systems and Technology (PAST), Oct 2016.

[39] M. Ibraheam, B. Bieske, K. Blau, E. Schäfer, A. Jäger, S. I. Butt, R. Stephan, and M. A. Hein, "Feasibility of dual-polarized antenna arrays for GNSS receivers at low elevations," in 11th European Conference on Antennas and Propagation, March 2017, pp. 857-861.

[40] W. H. Weedon and R. D. Nunes, "Low-cost wideband digital receiver/exciter (DREX) technology enabling next-generation all-digital phased arrays," in 2016 IEEE International Symposium on Phased Array Systems and Technology (PAST), Oct 2016, pp. 1-5.

[41] A. Valdes-Garcia, A. Natarajan, D. Liu, M. Sanduleanu, X. Gu, M. Ferriss, B. Parker, C. Baks, J. O. Plouchart, H. Ainspan, B. Sadhu, M. Islam, and S. Reynolds, "A fully-integrated dual-polarization 16-element W-band phasedarray transceiver in SiGe BiCMOS," in IEEE Radio Frequency Integrated Circuits Symposium, 2013, pp. 375-378.

[42] C. Fulton and W. J. Chappell, "Calibration of a digital phased array for polarimetric radar," in 2010 IEEE MTT-S International Microwave Symposium, May 2010, pp. 161-164.

[43] Y. Q. Wen, S. Gao, B. Z. Wang, and Q. Luo, "Dual-polarized and wideangle scanning microstrip phased array," IEEE Transactions on Antennas and Propagation, pp. 1-1, 2018.

[44] C. X. Mao, S. Gao, C. Tienda, T. Rommel, A. Patyuchenko, M. Younis, L. Boccia, E. Arnieri, S. Glisic, U. Yodprasit, P. Penkala, M. Krstic, F. Qin, O. Schrape, A. Koczor, G. Amendola, and V. Petrovic, "X/Ka-band dualpolarized digital beamforming synthetic aperture radar," IEEE Transactions on Microwave Theory and Techniques, vol. 65, no. 11, pp. 4400-4407, Nov 2017.

[45] S. B. Venkatakrishnan, E. A. Alwan, and J. L. Volakis, "Wideband RF selfinterference cancellation circuit for phased array simultaneous transmit and receive systems," IEEE Access, vol. 6, pp. 3425-3432, 2018. 
[46] C.-X. Mao, S. Gao, and T. Rommel, "Low-profile aperture-shared X/Ka-band dual-polarized antenna for DBF-SAR applications," in 2017 International Workshop on Antenna Technology: Small Antennas, Innovative Structures, and Applications (iWAT), March 2017, pp. 104-107.

[47] J. Hansen, D. J. Jung, and K. Chang, "Dual-polarized, X-band, flat-panel phased array," in Proceedings of the 2012 IEEE International Symposium on Antennas and Propagation, July 2012, pp. 1-2.

[48] P. Ahmadi, L. Belostotski, A. Madanayake, and J. W. Haslett, "0.96-to5.1GHz 4-element spatially analog IIR-enhanced delay-and-sum beamformer," in 2017 IEEE MTT-S International Microwave Symposium (IMS), June 2017, pp. $1610-1613$.

[49] A. Madanayake, V. Ariyarathna, N. Udayanga, L. Belostotski, S. K. Perera, and R. J. Cintra, "Design of a low-complexity wideband analog true-time-delay 5-beam array in 65nm CMOS," in 2017 IEEE 60th International Midwest Symposium on Circuits and Systems (MWSCAS), Aug 2017, pp. 1204-1207.

[50] V. Ariyarathna, N. Udayanga, A. Madanayake, S. M. Perera, L. Belostotski, and R. J. Cintra, "Design methodology of an analog 9-beam squint-free wideband IF multi-beamformer for mmW applications," in Moratuwa Engineering Research Conference, May 2017, pp. 236-240.

[51] C. Wijenayake, A. Madanayake, L. Belostotski, Y. Xu, and L. T. Bruton, "Linear RF apertures using 2-D analog beam filters," in IEEE International Symposium on Circuits and Systems, May 2016, pp. 293-296.

[52] P. Ahmadi, M. H. Taghavi, L. Belostotski, and A. Madanayake, "6 GHz allpass-filter-based delay-and-sum beamformer in 130nm CMOS," in 2014 IEEE 57th International Midwest Symposium on Circuits and Systems (MWSCAS), Aug 2014, pp. 837-840.

[53] C. Wijenayake, A. Madanayake, L. Belostotski, Y. Xu, and L. T. Bruton, "Allpass filter-based 2-D IIR filter-enhanced beamformers for AESA receivers," IEEE Transactions on Circuits and Systems I: Regular Papers, vol. 61, no. 5, pp. 1331-1342, May 2014.

[54] A. Madanayake, V. Ariyarathna, N. Akram, R. J. Cintra, and D. Coelho, "Multi-beam radio frequency $(\mathrm{RF})$ aperture arrays using multiplierless approximate fast Fourier transform (FFT)," University of Akron, United States, Tech. Rep., 2017. 
[55] A. Madanayake, R. J. Cintra, N. Akram, V. Ariyarathna, S. Mandal, V. A. Coutinho, F. M. Bayer, D. Coelho, and T. S. Rappaport, "Fast radix-32 approximate dfts for 1024-beam digital rf beamforming," IEEE Access, vol. 8, pp. 96 613-96 627, 2020.

[56] R. W. H. Jr, "Mimo at millimeter wave," Presentation, 2014. [Online]. Available: https://youtu.be/BQ45FuGpFQ0

[57] S. Hur, T. Kim, D. J. Love, J. V. Krogmeier, T. A. Thomas, and A. Ghosh, "Millimeter wave beamforming for wireless backhaul and access in small cell networks," IEEE Transactions on Communications, vol. 61, no. 10, pp. 43914403, 2013.

[58] Junyi Wang, Zhou Lan, Chang-woo Pyo, T. Baykas, Chin-sean Sum, M. A. Rahman, Jing Gao, R. Funada, F. Kojima, H. Harada, and S. Kato, "Beam codebook based beamforming protocol for multi-gbps millimeter-wave wpan systems," IEEE Journal on Selected Areas in Communications, vol. 27, no. 8, pp. 1390-1399, 2009.

[59] V. Ariyarathna, "Algorithms and circuits for analog-digital hybrid multibeam arrays," Ph.D. dissertation, Florida International University, 2019.

[60] O. E. Ayach, S. Rajagopal, S. Abu-Surra, Z. Pi, and R. W. Heath, "Spatially sparse precoding in millimeter wave MIMO systems," IEEE Transactions on Wireless Communications, vol. 13, no. 3, pp. 1499-1513, 2014.

[61] J. Xu, W. Xu, D. W. K. Ng, and A. L. Swindlehurst, "Secure communication for spatially sparse millimeter-wave massive MIMO channels via hybrid precoding," IEEE Transactions on Communications, vol. 68, no. 2, pp. 887-901, 2020 .

[62] A. Alkhateeb, O. El Ayach, G. Leus, and R. W. Heath, "Channel estimation and hybrid precoding for millimeter wave cellular systems," IEEE Journal of Selected Topics in Signal Processing, vol. 8, no. 5, pp. 831-846, 2014.

[63] D. T. P. Viswanath, Fundamentals of Wireless Communication. Cambridge University Printers, 2013.

[64] B. M. Hochwald, T. L. Marzetta, and V. Tarokh, "Multiple-antenna channel hardening and its implications for rate feedback and scheduling," IEEE Transactions on Information Theory, vol. 50, no. 9, pp. 1893-1909, 2004. 
[65] M. Alouzi and F. Chan, "Millimeter wave massive mimo with alamouti code and imperfect channel state information," in 2018 IEEE 5G World Forum (5GWF), 2018, pp. 507-511.

[66] T. L. Marzetta, Fundamentals of massive MIMO. Cambridge University Press, 2016.

[67] P. T. Kulkarni, M. H. Lee, and R. P. Paudel, "Performance analysis of dirtypaper coding over mimo keyhole channels," in 2006 International Conference on Electrical and Computer Engineering, 2006, pp. 402-407.

[68] T. L. Marzetta, "How much training is required for multiuser mimo?" in 2006 Fortieth Asilomar Conference on Signals, Systems and Computers, 2006, pp. 359-363.

[69] — - "Noncooperative cellular wireless with unlimited numbers of base station antennas," IEEE Transactions on Wireless Communications, vol. 9, no. 11, pp. 3590-3600, 2010.

[70] J. Hoydis, C. Hoek, T. Wild, and S. ten Brink, "Channel measurements for large antenna arrays," in 2012 International Symposium on Wireless Communication Systems (ISWCS), 2012, pp. 811-815.

[71] E. J. Black, "Holographic beam forming and mimo," Pivotal Commware, 2017.

[72] E. Björnson, "Holographic beamforming versus massive MIMO." [Online]. Available: https://ma-mimo.ellintech.se/2018/03/16/ holographic-beamforming-versus-massive-mimo/

[73] — - "The end of independent Rayleigh fading." [Online]. Available: https:// ma-mimo.ellintech.se/2020/10/15/the-end-of-independent-rayleigh-fading/

[74] E. Bjornson, J. Hoydis, and L. Sanguinetti, Massive MIMO Networks. now publishers Inc, 2017. [Online]. Available: https://www.ebook.de/de/product/31416100/emil_bjornson_jakob_ hoydis_luca_sanguinetti_massive_mimo_networks.html

[75] G. A. T. Warren L. Stutzman, Antenna Theory and Design. WILEY, 2012. [Online]. Available: https://www.ebook.de/de/product/16212802/ warren_l_stutzman_gary_a_thiele_antenna_theory_and_design.html 
[76] T. K. Gunaratne, "Beamforming of broadband bandpass signals using multidimensional fir filters," Ph.D. dissertation, University of Calgary, 2011.

[77] D. E. Dudgeon and R. M. Mersereau, Multidimensional Digital Signal Processing (Prentice-hall Signal Processing Series). Prentice Hall, 1983.

[78] I. Montesinos-Ortego, J. L. Masa-Campos, M. Sierra-Perez, and J. L. Fernandez-Jambrina, "Pyramidal adaptive antenna of plannar arrays for satellite comunications," in The Second European Conference on Antennas and Propagation, EuCAP 2007, 2007, pp. 1-5.

[79] T. Li, F. Zhang, F. Zhang, Y. Yao, and L. Jiang, "Wideband and high-gain uniform circular array with calibration element for smart antenna application," IEEE Antennas and Wireless Propagation Letters, vol. 15, pp. 230-233, 2016.

[80] L. C. Kretly, A. Cerqueira S, and A. Tavora AS, "A hexagonal antenna array prototype for adaptive system application," in The 5th International Symposium on Wireless Personal Multimedia Communications, vol. 2, 2002, pp. 757-761 vol.2.

[81] W. Gautier, W. Gruener, R. Rieger, and S. Chartier, "Broadband multifunction AESA front-ends: New requirements and emerging technologies," in 46th European Microwave Conference, 2016, pp. 1481-1484.

[82] C. Wijenayake, A. Madanayake, and L. Bruton, "Broadband multiple conebeam 3-D IIR digital filters applied to planar dense aperture arrays," IEEE Transactions on Antennas and Propagation, vol. 60, no. 11, pp. 5136-5146, Nov 2012.

[83] L. T. Bruton, "Three-dimensional cone filter banks," IEEE Transactions on Circuits and Systems I: Fundamental Theory and Applications, vol. 50, no. 2, pp. 208-216, Feb 2003.

[84] R. J. Mailloux, Phased Array Antenna Handbook. Artech House Publishers, 2017. [Online]. Available: https://www.ebook.de/de/product/29930846/ robert_j_mailloux_phased_array_antenna_handbook.html

[85] C. A. Balanis, Antenna Theory. Hoboken, NJ: Wiley John + Sons, 2016. [Online]. Available: https://www.ebook.de/de/product/24340252/ constantine_a_balanis_antenna_theory.html 
[86] H. Roger A. Horn, Topics in Matrix Analysis. Cambridge University Press, 2010. [Online]. Available: https://www.ebook.de/de/product/3303759/ roger_a_horn_horn_topics_in_matrix_analysis.html

[87] M. T. Heideman, "Multiplicative complexity of linear and bilinear systems," in Multiplicative Complexity, Convolution, and the DFT. Springer, 1988, pp. $5-26$.

[88] E. M. Deloraine, S. Van Mierlo, and B. Derjavitch, "Methode et systéme de transmission par impulsions," French Patent, vol. 932, p. 140, 1946.

[89] D. E. Maurice and D. Boris, "Communication system utilizing constant amplitude pulses of opposite polarities," Feb. 24 1953, uS Patent 2,629,857.

[90] F. De Jager and W. Jackson, "Delta modulation - a method of pcm transmission using the 1-unit code'," Communication theory, pp. 119-137, 1953.

[91] H. Van de Weg, "Quantizing noise of a single integration delta-modulation system with an n-digit code," Philips Research Reports, vol. 8, no. 5, pp. 367-385, 1953.

[92] C. C. Cutler, "Differential quantization of communication signals," Jul. 29 1952, uS Patent 2,605,361.

[93] B. E. Boser and B. A. Wooley, "The design of sigma-delta modulation analogto-digital converters," IEEE Journal of Solid-State Circuits, vol. 23, no. 6, pp. 1298-1308, Dec 1988.

[94] P. M. Aziz, H. V. Sorensen, and J. vn der Spiegel, "An overview of sigma-delta converters," IEEE Signal Processing Magazine, vol. 13, no. 1, pp. 61-84, Jan 1996.

[95] S. Handagala, A. Madanayake, L. Belostotski, and L. T. Bruton, " $\Delta-\Sigma$ noise shaping in 2D space-time for uniform linear aperture array receivers," in IEEE Moratuwa Engineering Research Conference, 2016.

[96] A. Nikoofard, J. Liang, M. Twieg, S. Handagala, A. Madanayake, L. Belostotski, and S. Mandal, "Low-complexity $n$-port ADCs using 2- $d \delta-\sigma$ noise-shaping for $n$-element array receivers," in 2017 IEEE 60th International Midwest Symposium on Circuits and Systems (MWSCAS), Aug 2017, pp. 301-304. 
[97] Y. Wang, S. Handagala, A. Madanayake, L. Belostotski, and S. Mandal, "Nport LNAs for mmW array processors using 2-D spatio-temporal $\delta$ - $\sigma$ noiseshaping," in 2017 IEEE 60th International Midwest Symposium on Circuits and Systems (MWSCAS), Aug 2017, pp. 1473-1476.

[98] R. Corey and A. Singer, "Spatial sigma-delta signal acquisition for wideband beamforming arrays," in 20th International ITG Workshop on Smart Antennas (WSA), March 2016.

[99] L. Bruton, "Low-sensitivity digital ladder filters," IEEE Transactions on Circuits and Systems, vol. 22, no. 3, pp. 168-176, Mar 1975.

[100] P. Agathoklis and L. T. Bruton, "Practical-bibo stability of n-dimensional discrete systems," IEE Proceedings G-Electronic Circuits and Systems, vol. 130, no. 6, pp. 236-242, December 1983.

[101] L. Bruton and N. Bartley, "Three-dimensional image processing using the concept of network resonance," IEEE Transactions on Circuits and Systems, vol. 32, no. 7, pp. 664-672, July 1985.

[102] H. L. P. A. Madanayake and L. T. Bruton, "Low-complexity distributed parallel processor for 2D IIR broadband beam plane-wave filters," Canadian Journal of Electrical and Computer Engineering, vol. 32, no. 3, pp. 123-131, Summer 2007.

[103] L. T. Bruton, A. Madanayake, C. Wijenayake, and M. Maini, "Continuoustime analog two-dimensional IIR beam filters," IEEE Transactions on Circuits and Systems II: Express Briefs, vol. 59, no. 7, pp. 419-423, July 2012.

[104] A. Zamanifekri, "Ka-band integrated focal-plane arrays for two-way satellite communication," Ph.D. dissertation, Department of Electrical Engineering, Jun. 2015, proefschrift. [Online]. Available: https://pure.tue.nl/ws/files/ 11271441/20151230_Zamanifekri.pdf

[105] P. J. Hall, The Square Kilometre Array: An Engineering Perspective. Springer, Dordrecht, 2005, ISBN 978-1-4020-3797-9.

[106] B. Veidt and P. Dewdney, "A phased-array feed demonstrator for radio telescopes," in In proceedings of URSI General Assembly, vol. 8, 2005, p. 84. 
[107] T. Oosterloo, M. Verheijen, W. van Cappellen, L. Bakker, G. Heald, and M. Ivashina, "The latest on apertif," Proceedings of Science, 2009. [Online]. Available: https://pos.sissa.it/089/006/pdf

[108] T. Oosterloo, M. Verheijen, W. van Cappellen, L. Bakker, M. Ivashina, and G. Heald, "Apertif-the focal-plane array system for the wsrt," in SKADS Conference, 2009.

[109] C. Cappellin, J. de Lasson, O. Iupikov, M. Ivashina, N. Skou, K. Pontoppidan, and B. Fiorelli, "Focal plane array breadboard for advanced multiple beam radiometr antennas," in 38th ESA Antenna Workshop on Innovative Antenna Systems and Technologies for Future Space Missions, 2017.

[110] M. V. Ivashina, J. G. bij de Vaate, R. Braun, and J. D. Bregman, "Focal plane arrays for large reflector antennas: first results of a demonstrator project," SPIE Proceedings, pp. 5489 - 5489 - 12, 2004. [Online]. Available: http://dx.doi.org/10.1117/12.554895

[111] L. T. Bruton, "Enel699 Multidimensional signal processing," 2004. [Online]. Available: http://www-mddsp.enel.ucalgary.ca/People/bruton/Enel699Main. html

[112] J. Liu, Z. Luo, and X. Xiong, "Low-resolution ADCs for wireless communication: A comprehensive survey," IEEE Access, vol. 7, pp. 91 291-91324, 2019 .

[113] B. S. Ramesh Harjani, Cognitive Radio Receiver Front-Ends. Springer New York, 2013.

[114] K. Greene, V. Chauhan, and B. Floyd, "Code-modulated embedded test for phased arrays," in 2016 IEEE 34th VLSI Test Symposium (VTS), 2016, pp. $1-4$.

[115] E. A. Alwan, A. Akhiyat, M. LaRue, W. Khalil, and J. L. Volakis, "Low cost, power efficient, on-site coding receiver (oscr) for ultra-wideband digital beamforming," in 2013 IEEE International Symposium on Phased Array Systems and Technology, Oct 2013, pp. 202-206.

[116] S. Bojja Venkatakrishnan, D. K. Papantonis, A. A. Akhiyat, E. A. Alwan, and J. L. Volakis, "Experimental validation of on-site coding digital beamformer with ultra-wideband antenna arrays," IEEE Transactions on Microwave Theory and Techniques, vol. 65, no. 11, pp. 4408-4417, Nov 2017. 
[117] S. B. Venkatakrishnan, A. Akhiyat, E. A. Alwan, W. Khalil, and J. L. Volakis, "Realization of a novel on-site coding digital beamformer using FPGAs," in 2014 USNC-URSI Radio Science Meeting (Joint with AP-S Symposium), July 2014, pp. 196-196.

[118] Y. C. Eldar, Sampling Theory. Cambridge University Press, 2017.

[119] "Walsh-Hadamard code." [Online]. Available: https://wiki.cse.buffalo.edu/ cse545/content/walsh-hadamard-code

[120] P. P. Vaidyanathan, Multirate systems and filter banks. Pearson Education India, 2006.

[121] S. Pulipati, V. Ariyarathna, U. De Silva, N. Akram, E. Alwan, A. Madanayake, S. Mandal, and T. S. Rappaport, "A direct-conversion digital beamforming array receiver with $800 \mathrm{MHz}$ channel bandwidth at $28 \mathrm{GHz}$ using Xilinx RF SoC," in 2019 IEEE International Conference on Microwaves, Antennas, Communications and Electronic Systems (COMCAS). IEEE, 2019, pp. 1-5.

[122] Analog Devices, GaAs MMIC I/Q Downconverter HMC1065LP4E. [Online]. Available: https://www.analog.com/media/en/technical-documentation/ data-sheets/hmc1065.pdf

[123] D. C. Champeney, A Handbook of Fourier Theorems. Cambridge University Press, 1987.

[124] A. V. Oppenheim and R. W. Schafer, Discrete-time Signal Processing, 3rd ed. Prentice Hall, 2009.

[125] R. E. Blahut, Fast Algorithms for Signal Processing. Cambridge University Press, 2010.

[126] C. Yang, Y. Xie, L. Chen, H. Chen, and Y. Deng, "Design of a configurable fixed-point FFT processor," in IET International Radar Conference 2015, Oct 2015, pp. 1-4.

[127] C. V. Kumar and K. R. K. Sastry, "Design and implementation of FFT pruning algorithm on FPGA," in 7th International Conference on Cloud Computing, Data Science Engineering - Confluence, Jan 2017, pp. 739-743. 
[128] T. Ayhan, W. Dehaene, and M. Verhelst, "A 128:2048/1536 point FFT hardware implementation with output pruning," in 22nd European Signal Processing Conference (EUSIPCO), Sep. 2014, pp. 266-270.

[129] M. Z. A. Khan and S. Qadeer, "A new variant of radix-4 FFT," in 13th International Conference on Wireless and Optical Communications Networks (WOCN), July 2016, pp. 1-4.

[130] S. Zhang, A. A. Joseph, D. Voit, S. Schaetz, K.-D. Merboldt, C. UnterbergBuchwald, A. Hennemuth, J. Lotz, and J. Frahm, "Real-time magnetic resonance imaging of cardiac function and flow - recent progress," Quantitative Imaging in Medicine and Surgery, vol. 4, no. 5, p. 313, 2014.

[131] L. Axel and R. Otazo, "Accelerated MRI for the assessment of cardiac function," The British Journal of Radiology, vol. 89, no. 1063, p. 20150655, 2016.

[132] D. M. Suárez Villagrán, "Aproximações para a transformada discreta de Fourier e aplicações em deteção e estimação," Master's thesis, Universidade Federal de Pernambuo, Recife, Brazil, 2015, advisors: R. J. Cintra and F. M. Bayer.

[133] A. Madanayake, V. Ariyarathna, S. Madishetty, S. Pulipati, R. J. Cintra, D. Coelho, R. Oliveira, F. Bayer, L. Belostotski, and S. M. T. Rappaport, "Towards a low-SWaP 1024-beam digital array: A 32-beam sub-system at 5.8 GHz," IEEE Transactions on Antennas \&3 Propagation, 2018, to Appear.

[134] K. R. Rao and P. C. Yip, Eds., The Transform and Data Compression Handbook, ser. Electrical Engineering \& Applied Signal Processing Series. CRC Press, 2000.

[135] J. W. Cooley and J. W. Tukey, "An algorithm for the machine calculation of complex Fourier series," Mathematics of Computation, vol. 19, no. 90, pp. 297-301, 1965. [Online]. Available: http://www.jstor.org/stable/2003354

[136] P. Duhamel and H. Hollmann, "Split radix FFT algorithm," Electronics Letters, vol. 20, no. 1, pp. 14-16, 1984.

[137] S. Winograd, "On computing the discrete Fourier transform," Mathematics of computation, vol. 32, no. 141, pp. 175-199, 1978.

[138] — "On the multiplicative complexity of the discrete Fourier transform," Advances in Mathematics, vol. 32, no. 2, pp. 83-117, 1979. 
[139] — Arithmetic Complexity of Computations. CBMS-NSF Regional Conference Series in Applied Mathematics, 1980.

[140] S. G. Johnson and M. Frigo, "A modified split-radix FFT with fewer arithmetic operations," IEEE Transactions on Signal Processing, vol. 55, no. 1, pp. 111119, 2007.

[141] T. Duong, "vec, vech, invvec, invvech," https://www.rdocumentation.org/, 2019.

[142] MathWorks, "MATLAB R2018b documentation "vec2mat"," https://www.mathworks.com/help/comm/ref/vec2mat, 2019.

[143] - - "MATLAB R2018b documentation "reshape"," https://www.mathworks.com/help/matlab/ref/reshape, 2019.

[144] G. A. F. Seber, A Matrix Handbook for Statisticians, ser. Wiley Series in Probability and Statistics. Hoboken, NJ: John Wiley \& Sons, Inc., 2008.

[145] D. Suarez, R. J. Cintra, F. M. Bayer, A. Sengupta, S. Kulasekera, and A. Madanayake, "Multi-beam RF aperture using multiplierless FFT approximation," Electronics Letters, vol. 50, no. 24, pp. 1788-1790, 2014.

[146] S. Kulasekera, A. Madanayake, R. J. Cintra, D. Suarez, and F. M. Bayer, "Multi-beam receiver apertures using multiplierless 8-point approximate DFT," in IEEE Radar Conference (RadarCon), 2015.

[147] S. Noh, Y. Jung, S. Lee, and J. Kim, "Low-complexity symbol detector for MIMO-OFDM-based wireless LANs," IEEE Transactions on Circuits and Systems II: Express Briefs, vol. 53, no. 12, pp. 1403-1407, Dec 2006.

[148] T. Demeechai, T. Chang, and S. Siwamogsatham, "Performance of a frequency-domain OFDM-timing estimator," IEEE Communications Letters, vol. 16, no. 10, pp. 1680-1683, October 2012.

[149] A. M. Jaradat, J. M. Hamamreh, and H. Arslan, "Modulation options for OFDM-based waveforms: Classification, comparison, and future directions," IEEE Access, vol. 7, pp. 17 263-17 278, 2019.

[150] M. N. and, "A simple and effective post-FFT beamforming technique for QAM-OFDM systems," in 2007 International Symposium on Microwave, An- 
tenna, Propagation and EMC Technologies for Wireless Communications, Aug 2007, pp. 70-73.

[151] A. Schwarzinger, Digital Signal Processing in Modern Communication Systems. Andreas Schwarzinger, 2013.

[152] R. Deng, J. He, M. Chen, Y. Liu, and L. Chen, "Real-time lr-ddo-ofdm transmission system using eml with 1024-point fft," IEEE Photonics Technology Letters, vol. 27, no. 17, pp. 1841-1844, 2015.

[153] B. Inan, O. Karakaya, P. Kainzmaier, S. Adhikari, S. Calabro, V. A. J. M. Sleiffer, N. Hanik, and S. L. Jansen, "Realization of a $23.9 \mathrm{gb} / \mathrm{s}$ real time optical-ofdm transmitter with a 1024 point ifft," in 2011 Optical Fiber Communication Conference and Exposition and the National Fiber Optic Engineers Conference, March 2011, pp. 1-3.

[154] B. Inan, S. Adhikari, O. Karakaya, P. Kainzmaier, M. Mocker, H. von Kirchbauer, N. Hanik, and S. L. Jansen, "Realization of a real-time 93.8-Gb/s polarization-multiplexed OFDM transmitter with 1024-point IFFT," in 2011 37th European Conference and Exhibition on Optical Communication, Sep. 2011, pp. 1-3.

[155] W. Kabir, "Orthogonal frequency division multiplexing (ofdm)," in 2008 China-Japan Joint Microwave Conference, 2008, pp. 178-184.

[156] B. Sklar, Digital Communications: Fundamentals and Applications (2nd Edition). Prentice Hall, 2017.

[157] S. W. Ellingson and W. Cazemier, "Efficient multibeam synthesis with interference nulling for large arrays," IEEE Transactions on Antennas and Propagation, vol. 51, no. 3, pp. 503-511, March 2003.

[158] J. O. Coleman, "A generalized FFT for many simultaneous receive beams," Naval Research Lab, Washington DC - Signal Processing Section, Tech. Rep., 2007.

[159] M. Hossain, K. Nosaeva, B. Janke, N. Weimann, V. Krozer, and W. Heinrich, "A G-band high power frequency doubler in transferred-substrate InP HBT technology," IEEE Microwave and Wireless Components Letters, vol. 26, no. 1, pp. 49-51, Jan 2016. 
[160] K. Zhou, J. Ding, C. Zhou, and W. Wu, "W-band dual-band quasi-elliptical waveguide filter with flexibly allocated frequency and bandwidth ratios," IEEE Microwave and Wireless Components Letters, vol. 28, no. 3, pp. 206-208, March 2018.

[161] A. Kurdoghlian, H. Moyer, H. Sharifi, D. F. Brown, R. Nagele, J. Tai, R. Bowen, M. Wetzel, R. Grabar, D. Santos, and M. Micovic, "First demonstration of broadband W-band and D-band GaN MMICs for next generation communication systems," in 2017 IEEE MTT-S International Microwave Symposium (IMS), June 2017, pp. 1126-1128.

[162] W. Shaobing, G. Jianfeng, W. Weibo, and Z. Junyun, "W-band MMIC PA with ultrahigh power density in 100-nm AlGaN/GaN technology," IEEE Transactions on Electron Devices, vol. 63, no. 10, pp. 3882-3886, Oct 2016.

[163] S. Carpenter, Z. S. He, and H. Zirath, "Balanced active frequency multipliers in D and G bands using 250nm InP DHBT technology," in IEEE Compound Semiconductor Integrated Circuit Symposium (CSICS), Oct 2017, pp. 1-4.

[164] M. Rodwell, Z. Griffith, N. Parthasarathy, E. Lind, C. Sheldon, S. R. Bank, U. Singisetti, M. Urteaga, K. Shinohara, R. Pierson, and P. Rowell, "Developing bipolar transistors for sub-mm-wave amplifiers and next-generation (300 GHz) digital circuits," in 64th Device Research Conference, June 2006, pp. $5-8$.

[165] J. Kim and J. F. Buckwalter, "Staggered gain for 100+ GHz broadband amplifiers," IEEE Journal of Solid-State Circuits, vol. 46, no. 5, pp. 1123-1136, May 2011.

[166] A. Natarajan, A. Komijani, X. Guan, A. Babakhani, and A. Hajimiri, "A 77GHz phased-array transceiver with on-chip antennas in silicon: Transmitter and local lo-path phase shifting," IEEE Journal of Solid-State Circuits, vol. 41, no. 12, pp. 2807-2819, 2006.

[167] A. Babakhani, X. Guan, A. Komijani, A. Natarajan, and A. Hajimiri, "A 77GHz phased-array transceiver with on-chip antennas in silicon: Receiver and antennas," IEEE Journal of Solid-State Circuits, vol. 41, no. 12, pp. 27952806, Dec 2006.

[168] V. Ariyarathna, D. F. G. Coelho, S. Pulipati, R. J. Cintra, F. M. Bayer, V. S. Dimitrov, and A. Madanayake, "Multibeam digital array receiver using a 16- 
point multiplierless DFT approximation," IEEE Transactions on Antennas and Propagation, vol. 67, no. 2, pp. 925-933, Feb 2019.

[169] V. A. Coutinho, V. Ariyarathna, D. F. G. Coelho, R. J. Cintra, and A. Madanayake, "An 8-beam $2.4 \mathrm{GHz}$ digital array receiver based on a fast multiplierless spatial DFT approximation," in 2018 IEEE/MTT-S International Microwave Symposium - IMS, June 2018, pp. 1538-1541.

[170] V. Ariyarathna, S. Kulasekera, A. Madanayake, K. Lee, D. Suarez, R. J. Cintra, F. M. Bayer, and L. Belostotski, "Multi-beam 4 GHz microwave apertures using current-mode DFT approximation on $65 \mathrm{~nm}$ CMOS," in 2015 IEEE MTT-S International Microwave Symposium, May 2015, pp. 1-4. 
VITA

\section{MOHOMED NAJATH AKRAM}

April 3, 1991

Born, Galle, Sri Lanka

2016

B.S., Engineering

University of Ruhuna

Galle, Sri Lanka.

\section{PUBLICATIONS AND PRESENTATIONS}

[J3] Najath Akram et al. "Digital Arrays with Reduced ADC Counts using SpaceTime Linear Transformation." IEEE Transactions on Circuits and Systems-II: Under Review.

[J2] Najath Akram et al. "Frequency-Multiplexed Array Digitization for MIMO Receivers: 4-Antennas/ADC at $28 \mathrm{GHz}$ on Xilinx ZCU-1285 RF SoC." IEEE Transactions on Antennas and Propagation: Under Review.

[J1] Arjuna Madanayake, Renato J. Cintra, Najath Akram et al. "Fast Radix-32 Approximate DFTs for 1024-Beam Digital RF Beamforming." IEEE Access (2020).

[C7] Najath Akram et al. "Massive-MIMO and Digital mm-Wave Arrays on RFSoCs using FDM for M-Fold Increase in Antennas per ADC/DAC," in IEEE Radio \& Wireless Week, 2020: Accepted

[C6] Arjuna Madanayake, Najath Akram et al. "A Four-Element Digital Array Receiver at $2.4 \mathrm{GHz}$ Using a Single Frequency-Multiplexed ADC" in IEEE AP-S Symposium on Antennas and Propagation and CNC/USNC-URSI, 2019.

[C5] Sravan Pulipati, Viduneth Ariyarathna, Najath Akram et al. "Design of 28 GHz 64-QAM digital receiver," in International Workshop on Antenna Technology (iWAT), pp. 193-196, 2019.

[C4] Sravan Pulipati, Viduneth Ariyarathna, Najath Akram et al. "A DirectConversion Digital Beamforming Array Receiver with $800 \mathrm{MHz}$ Channel Bandwidth at $28 \mathrm{GHz}$ using Xilinx RF SoC," in IEEE International Conference on Microwaves, Antennas, Communications and Electronic Systems (COMCAS), pp. 1-5, 2019.

[C3] Najath Akram et al. "Multiport ADCs for microwave focal plane array dish receivers," in IEEE International Symposium on Circuits and Systems (ISCAS), pp. 1-5, 2018. 
[C2] Arjuna Madanayake, Najath Akram et al. "Sampling H-\& V-Polarized Antennas using a Single ADC for Digital Antenna Arrays by Exploiting MultiDimensional Signal Processing RF Circuits," in IEEE $23^{\text {rd }}$ International Conference on Digital Signal Processing (DSP), pp. 1-5, 2018.

[C1] Arjuna Madanayake, Najath Akram et al. "Improving ADC figure-of-merit in wideband antenna array receivers using multidimensional space-time deltasigma multiport circuits," in $10^{\text {th }}$ International Workshop on Multidimensional Systems, pp. 1-6, 2017. 